\title{
SELECTION FOR RAPID MANUFACTURING UNDER EPISTEMIC UNCERTAINTY
}

\author{
A Thesis \\ Presented to \\ The Academic Faculty \\ By \\ Jamal Omari Wilson \\ In Partial Fulfillment \\ of the Requirements for the Degree \\ Master of Science in Mechanical Engineering
}

Georgia Institute of Technology

May 2006 


\section{SELECTION FOR RAPID MANUFACTURING UNDER EPISTEMIC UNCERTAINTY}

Approved by:

David W. Rosen, Chair

Professor, Mechanical Engineering

Georgia Institute of Technology

Chris J. J. Paredis

Assistant Professor, Mechanical Engineering

Georgia Institute of Technology

Janet K. Allen

Associate Professor, Mechanical

Engineering

Georgia Institute of Technology

Date Approved: 


\section{ACKNOWLEDGEMENTS}

W.B. Yeats, “...education is not the filling of a pail, but the lighting of a fire."

It is this fire of education that has driven me to pursue my higher education, and the completion of this Masters Thesis. It must be recognized that this fire does not come out of the sky, but must be initially ignited by those around us that have impact on our lives. With that, I want to begin by sincerely thanking those that have truly ignited this fire within me, my parents, Roger and Deborah Wilson. Ever since youth, my parents, who have both been afforded the opportunity of education, have instilled the value within me. I thank you mom and dad for all the inspiration and guidance, whether directly or indirectly, you have given me to complete this thesis. I also thank my brother, Rashad, and my girlfriend, Marilyn Simmons, for their love, support, and understanding throughout this process.

Most importantly, I would like to thank my Lord and Savior Jesus Christ for through Him and with his strength, all things can be achieved. It is only when our faith is tested that we truly realize his power.

I would like to thank my advisor, Dr. David Rosen, for his guidance and knowledge in completing this thesis. Not only had he initially taken a chance on me, but had enough faith in me to let me explore a topic that I perceived value in. It is only through this maze of exploration that learning takes place. 
I also must thank my reading committee, Dr. Janet Allen and Dr. Chris Paredis, for their guidance in completing this thesis. Each provided useful advice to help strengthen this thesis.

The students of the Systems Realization Laboratory have also contributed significantly to the completion of this thesis. I would especially like to thank Marco Fernandez, Benay Sager, Sundiata Jangha, and Christopher Williams for their peer guidance and helpful hand in completing this thesis.

Last but not least, this thesis could not have been completed without the financial support of the NASA Harriett G. Jenkins Predoctoral Fellowship Program and the David and Lucille Packard Foundation Fellowship. 


\section{TABLE OF CONTENTS}

ACKNOWLEDGEMENTS ...................................................................................ii

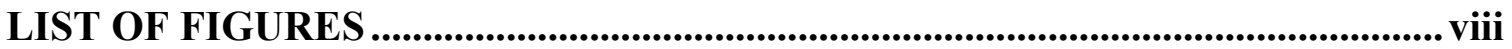

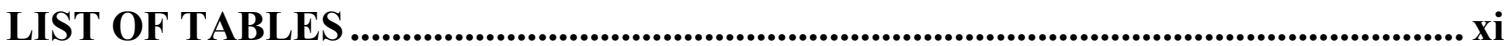

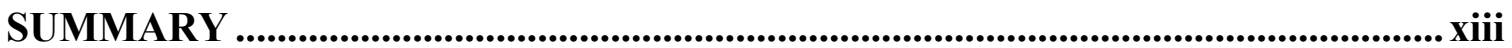

CHAPTER 1 CUSTOMIZATION AND RAPID MANUFACTURING .................... 1

1.1 BACKGROUND AND MOTIVATION FOR RESEARCH .................................. 1

1.2 RESEARCH PROBLEM AND CURRENT APPROACHES .................................5

1.3 RESEARCH GAP, QUESTIONS, AND HYPOTHESES ....................................8

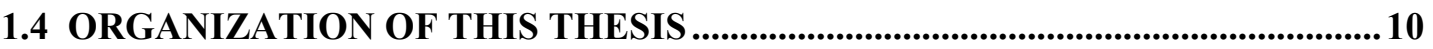

1.5 THE VALIDATION AND VERIFICATION STRATEGY FOR THIS THESIS .. 11

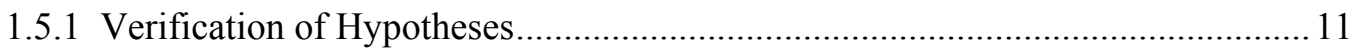

1.5.2 Validation of Selection for Rapid Manufacturing .............................................. 13

CHAPTER 2 THEORETICAL FOUNDATION: ELEMENTS OF SELECTION

FOR RAPID MANUFACTURING ...................................................................... 17

2.1 THE SELECTION DECISION SUPPORT PROBLEM ........................................... 17

2.1.1 Description, Word Formulation, and Mathematical Formulation ........................ 17

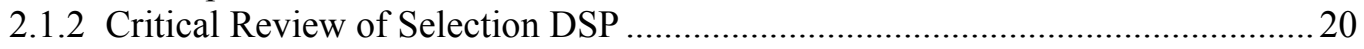

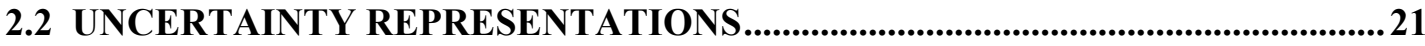

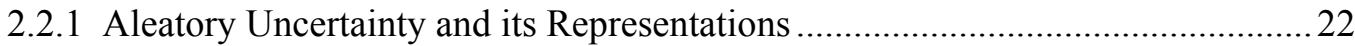

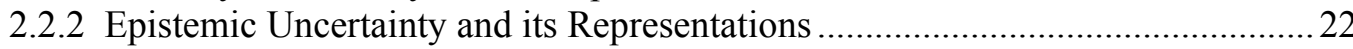

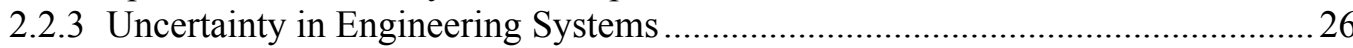

2.2.4 Critical Review of Uncertainty Handling Representations (Interval Analysis vs.

Probability Theory)

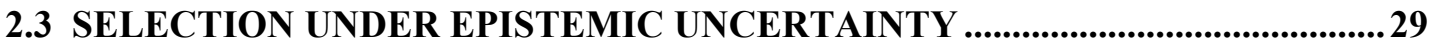

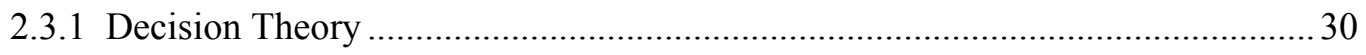

2.3.2 Four Selection Criteria for Strict Uncertainty .................................................... 31

2.3.3 Critical Review of Four Selection Criteria........................................................... 35

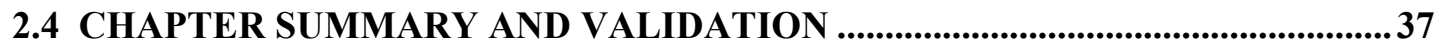




\section{CHAPTER 3 SYNTHESIZING THE CONSTRUCTS OF SELECTION FOR}

RAPID MANUFACTURING UNDER EPISTEMIC UNCERTAINTY.

3.1 UNCERTAINTY AND RAPID MANUFACTURING 39

3.2 A METHOD FOR SELECTION FOR RAPID MANUFACTURING .................... 40

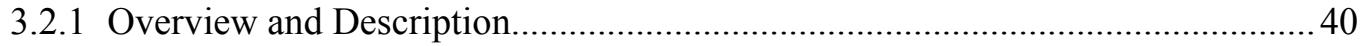

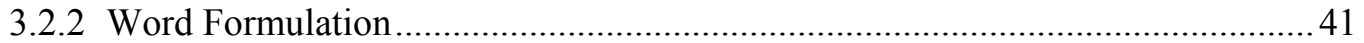

3.2.3 Steps for Selection for Rapid Manufacturing under epistemic uncertainty.......... 42

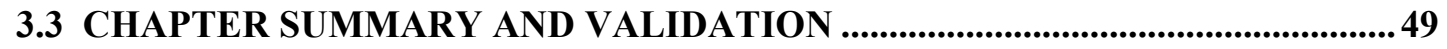

CHAPTER 4 BUILD TIME AND PART COST ESTIMATION MODELS.......... 50

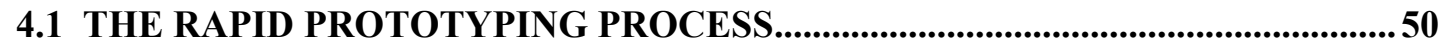

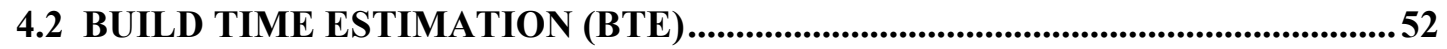

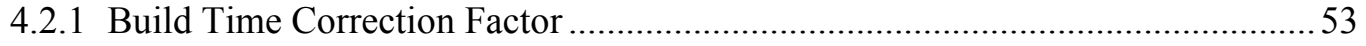

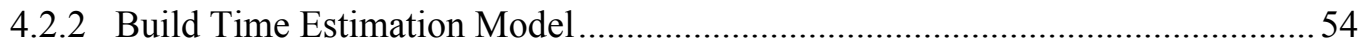

4.2.3 Build Time Estimation for Rapid Manufacturing …............................................56

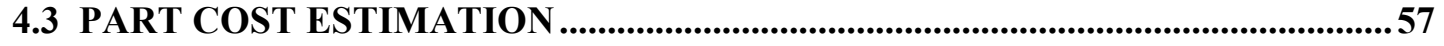

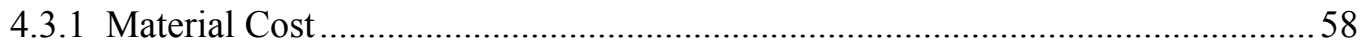

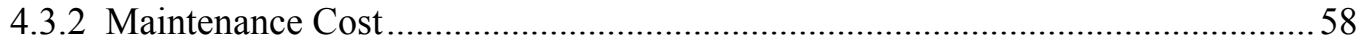

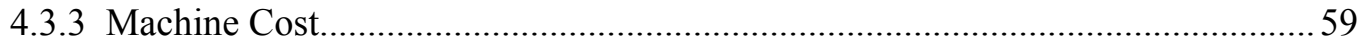

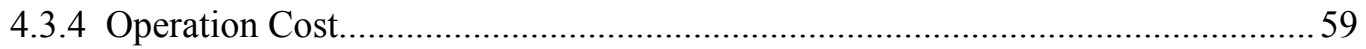

4.4 SPECIFIC RM BUILD TIME AND PART COST MODELS ..............................60

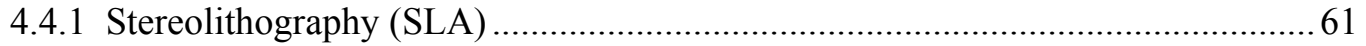

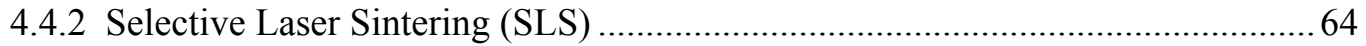

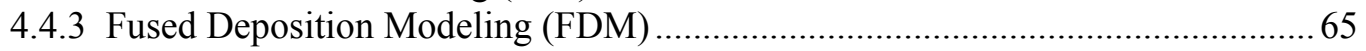

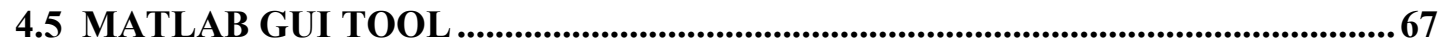

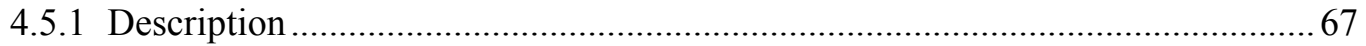

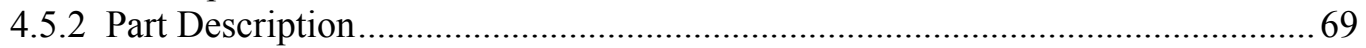

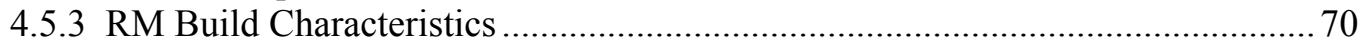

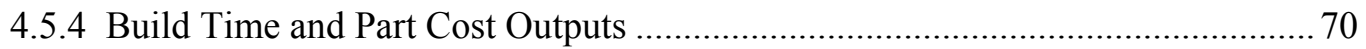

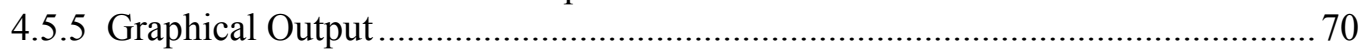

4.6 TESTING THE VALIDITY OF THE BUILD TIME AND COST ESTIMATION

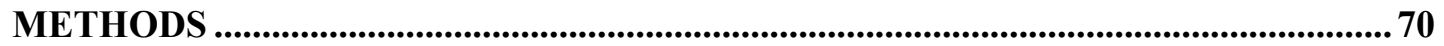

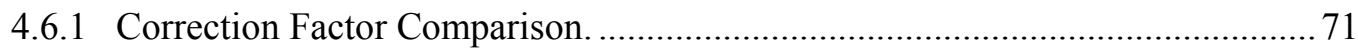

4.6.2 Quantitative Evaluation of the Build Time Estimator using SLS ....................... 72

4.6.3 Quantitative Evaluation of the Build Time Estimator using SLA .........................73

4.6.4 Qualitative Comparison of Build Time and Cost ............................................... 82

4.7 ADVANTAGES/ LIMITATIONS OF BUILD TIME AND PART COST

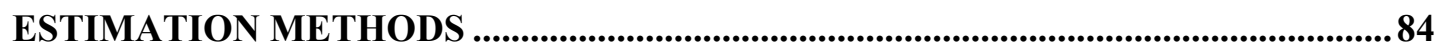

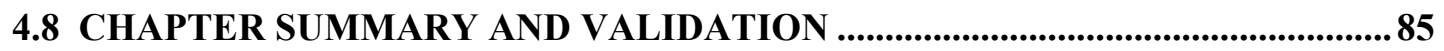


CHAPTER 5 ILLUSTRATIVE EXAMPLES.............................................................. 87

5.1 ILLUSTRATIVE EXAMPLE: DIRECT PRODUCTION OF CASTER WHEELS

........................................................................................................................................... 87

5.1.1 Albion and Rapid Manufacturing …………………………………………..... 88

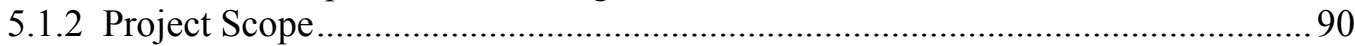

5.1.3 RM Technology Requirements .....................................................................95

5.1.4 Selection for Rapid Manufacturing ……………………………………………....96

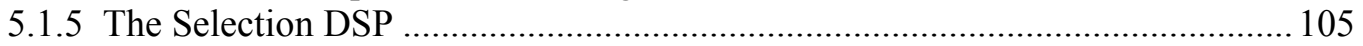

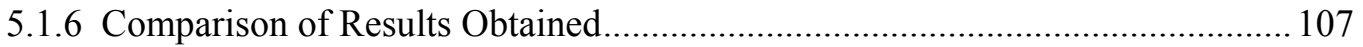

5.2 ILLUSTRATIVE EXAMPLE: DIRECT PRODUCTION OF HEARING AID

SHELLS ................................................................................................................110

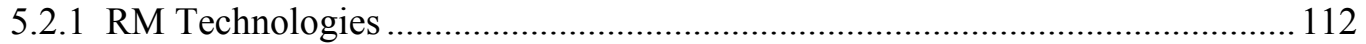

5.2.2 Selection for Rapid Manufacturing ……………………………………....... 112

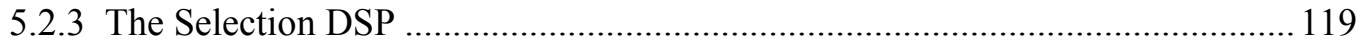

5.2.4 Comparison of Results Obtained........................................................................ 121

5.3 ADDITIONAL DISCUSSION OF SELECTION FOR RM .....................................123

5.4 DISCUSSION OF SELECTION CRITERIA...............................................................125

5.5 CHAPTER SUMMARY AND VALIDATION ………………………….......................126

CHAPTER 6 CLOSURE AND CONTRIBUTIONS .............................................. 129

6.1 REVISITING THE RESEARCH QUESTIONS.........................................................129

6.2 VALIDATION AND VERIFICATION ...................................................................132

6.3 REVIEW OF RESEARCH GAP AND CONTRIBUTIONS ………...........................135

6.4 RESEARCH LIMITATIONS AND FUTURE WORK ……........................................ 137

6.5 CLOSING REMARKS................................................................................................. 139

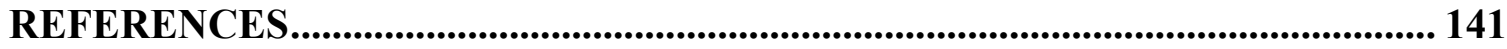




\section{LIST OF FIGURES}

Figure 1.1 The Economic Implications of Mass Customization ${ }^{1}$................................. 1

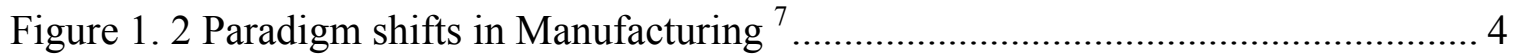

Figure 1. 3 Design Method Validation: A Process of Building Confidence in Usefulness

12

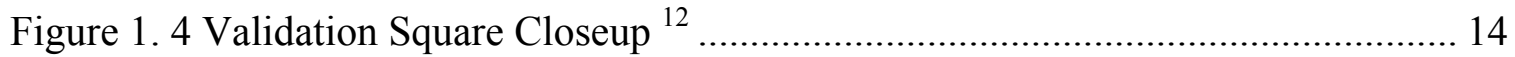

Figure 2. 1 The Selection Decision Support Problem [16] ....................................... 18

Figure 2. 2 Summary of Steps for selection Decision Support Problem [14]................. 18

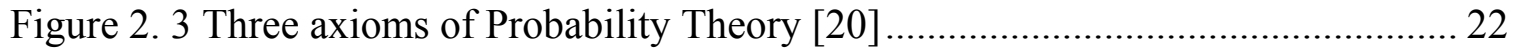

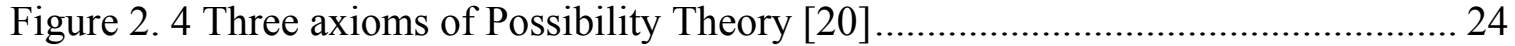

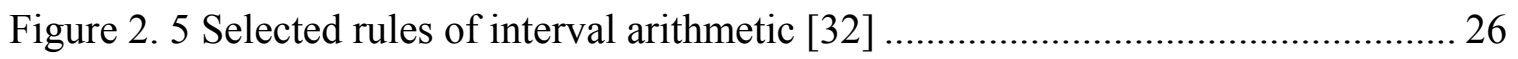

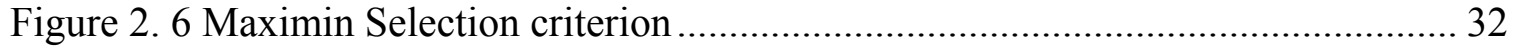

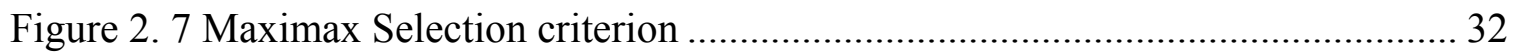

Figure 2. 8 Certainty equivalent determination ................................................. 33

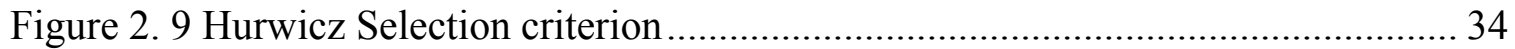

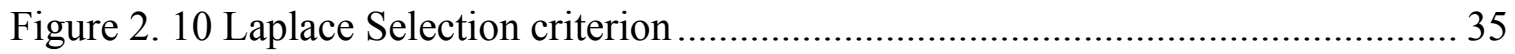

Figure 3. 1 Selection for RM Word Formulation ......................................................... 42

Figure 3. 2 Summary of Steps for Selection for Rapid Manufacturing ......................... 42

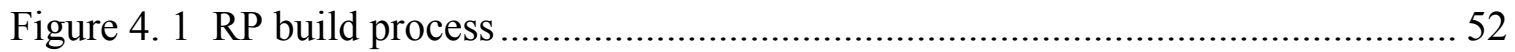

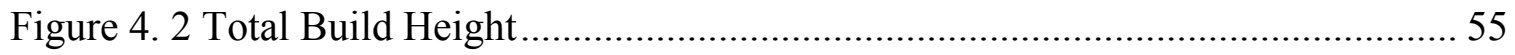


Figure 4. 3 Parametric Cost Model ........................................................................... 57

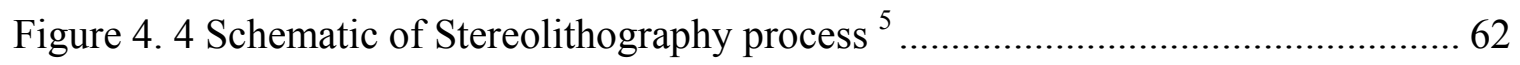

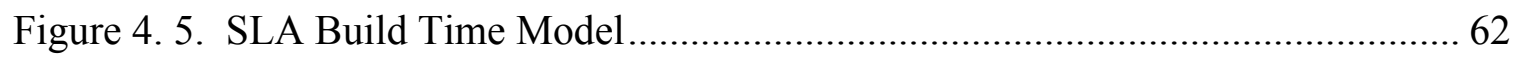

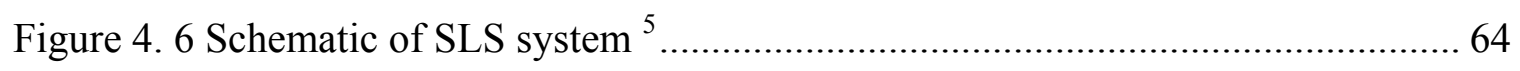

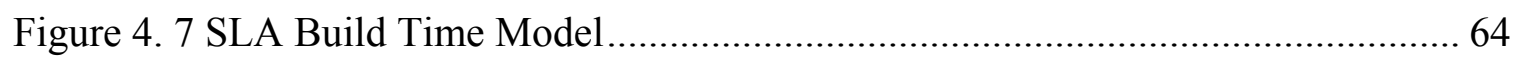

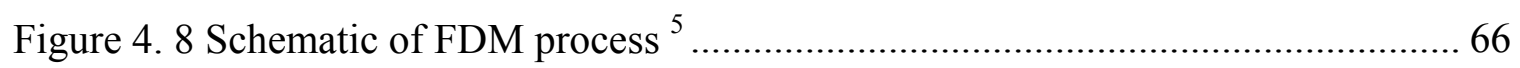

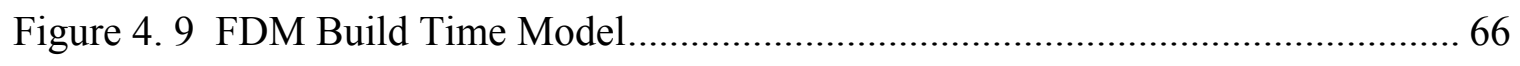

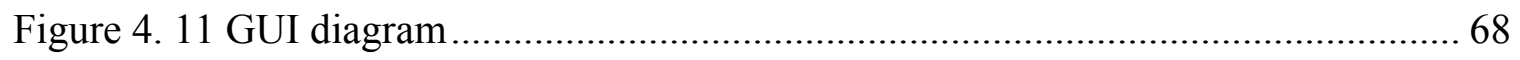

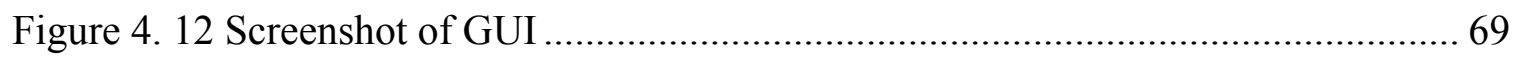

Figure 4. 13 Cross Sections for Example 1..................................................... 71

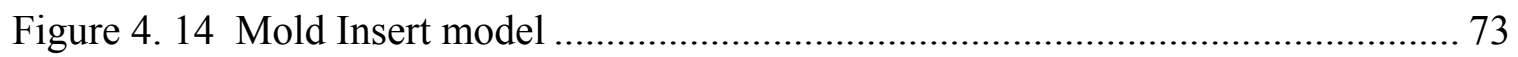

Figure 4. 15 Part 1 for multiple part evaluation..................................................... 75

Figure 4. 16 Part 2 for multiple part evaluation......................................................... 75

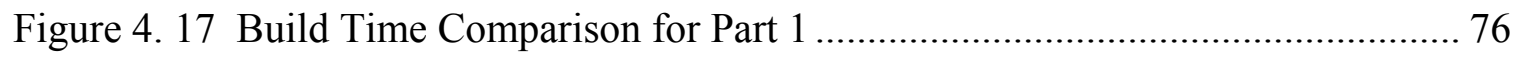

Figure 4. 18 Build Time Comparison for Part 2 …............................................... 76

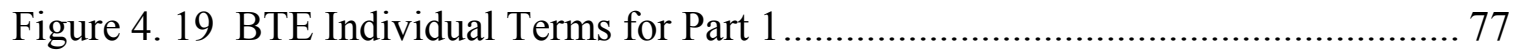

Figure 4. 20 BTE Individual terms for Part 2 ..................................................... 77

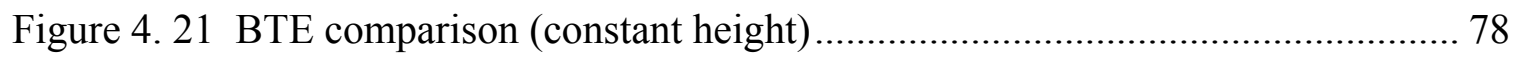

Figure 4. 22 Part Cost Estimation for Part 1 ........................................................ 79

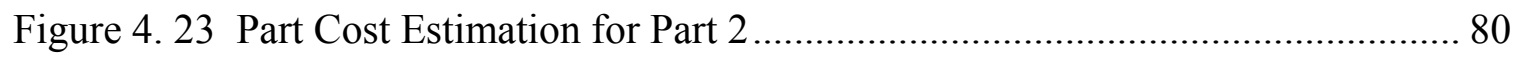

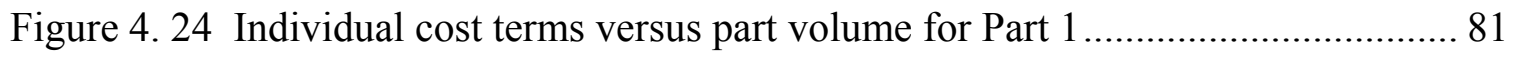

Figure 4. 25 Individual cost terms versus part volume for Part $2 \ldots \ldots \ldots \ldots \ldots \ldots \ldots \ldots \ldots \ldots \ldots . . . . . \ldots 1$

Figure 4. 26 Screenshot of Inputs and Outputs for qualitative comparison.................... 83 


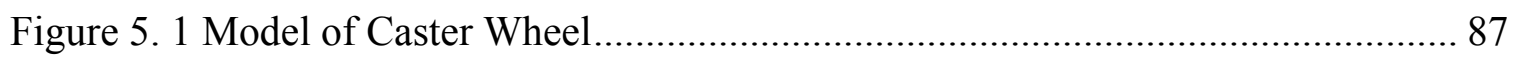

Figure 5. 2 Commercially Available Metal RM Technologies........................................... 91

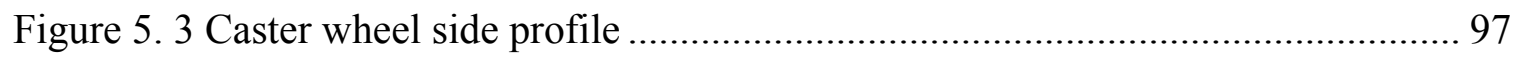

Figure 5. 4 Hurwicz evaluation parameter, $P(\alpha)$, as a function of Hurwicz Factor, $\alpha$ 104

Figure 5. 5 Merit Value as a function of uncertainty .................................................. 108

Figure 5. 6 Hearing Aid Shell [40] ..................................................................... 111

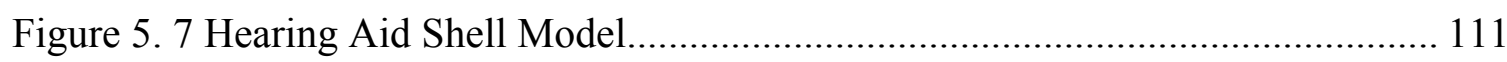

Figure 5. 8. Hurwicz evaluation parameter, $P(\alpha)$ as a function of Hurwicz Factor, $\alpha$

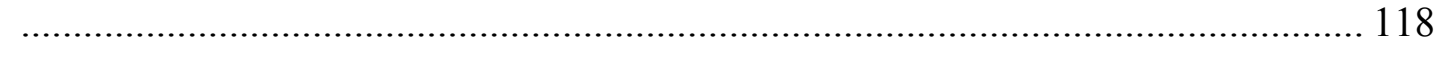

Figure 5. 9 Merit Value as a function of uncertainty ................................................. 122 


\section{LIST OF TABLES}

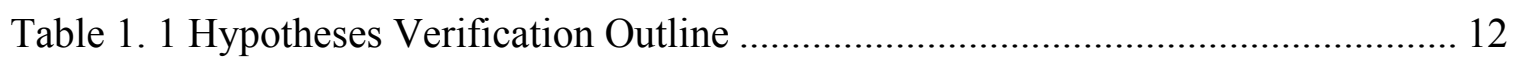

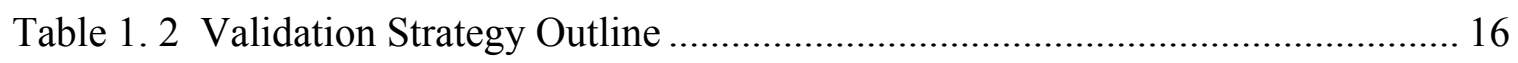

Table 2. 1 Possible scenarios of interval relations ...................................................... 29

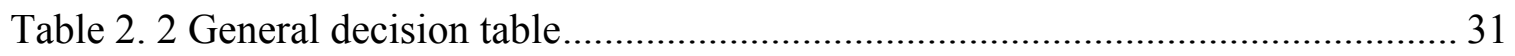

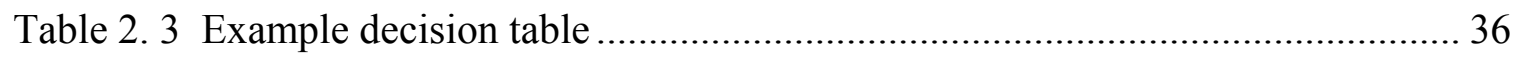

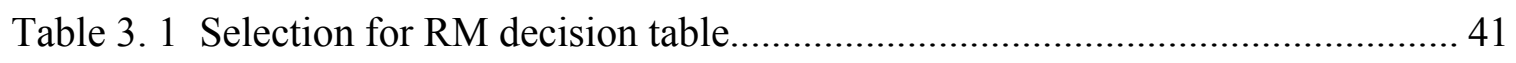

Table 3. 2 Eight Generic Levels of Customization ..................................................... 43

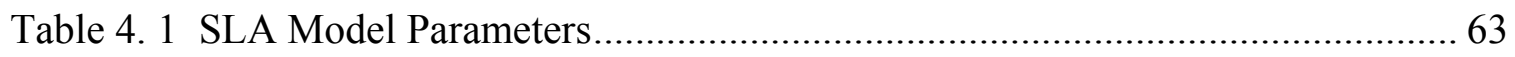

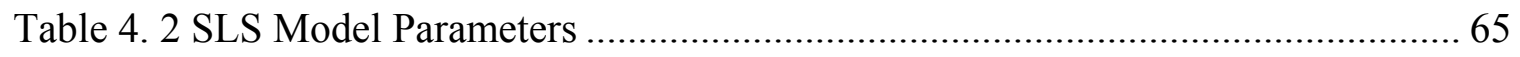

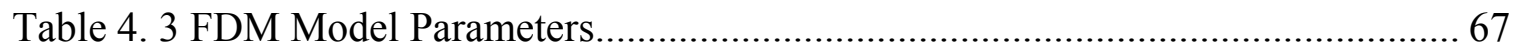

Table 4. 4 Build Time Results for Pham's factor comparison..................................... 72

Table 4. 5 Build time Part and Machine Characteristics ........................................... 72

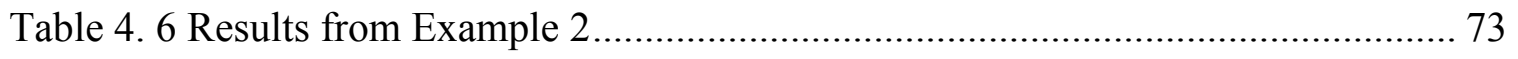

Table 4. 7 Estimated and Actual build time comparison ........................................... 74

Table 5. 1 Table of Commercially-Available RM Technologies for Metal ................... 91

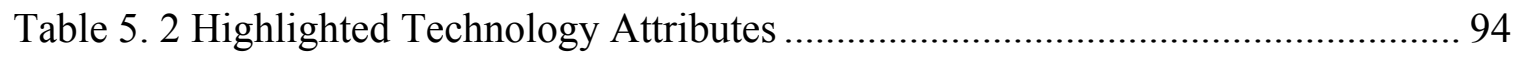

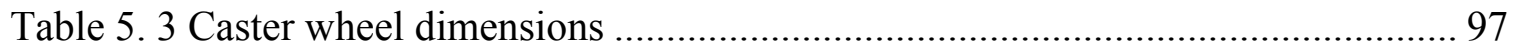




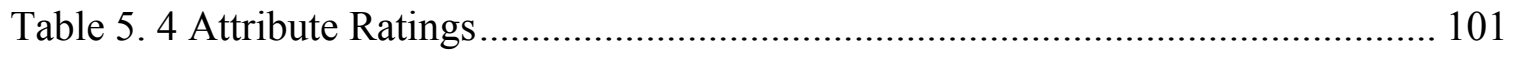

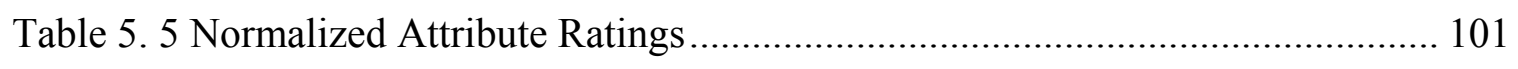

Table 5. 6 Alternative Merit Function Values for Scenario 1 and 2........................... 102

Table 5. 7 Selection parameters for Decision Theory selection criteria (Scenario 1) .... 102

Table 5. 8 Selection parameters for Decision Theory selection criteria (Scenario 2) ... 103

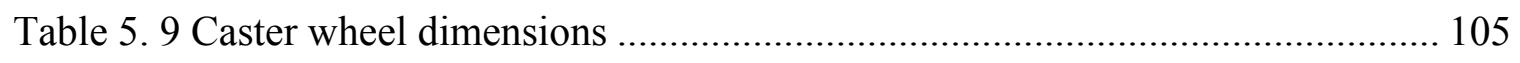

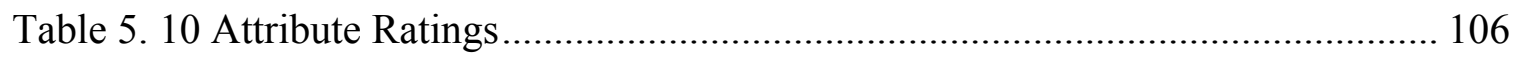

Table 5. 11 Merit Function Values ....................................................................... 107

Table 5. 12 Hearing Aid Shell Dimensions ............................................................. 113

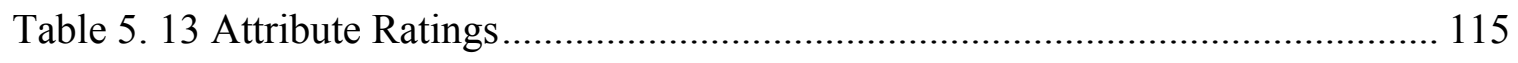

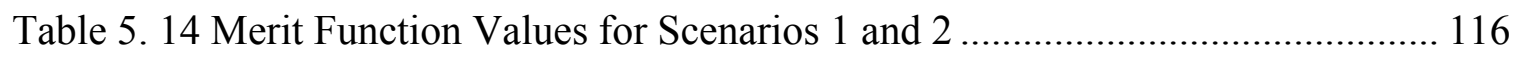

Table 5. 15 Selection Parameters for Scenario 1 ..................................................... 116

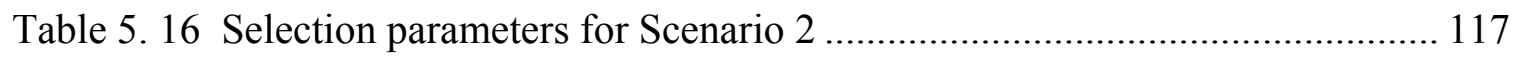

Table 5. 17 Hearing Aid shell dimensions .......................................................... 119

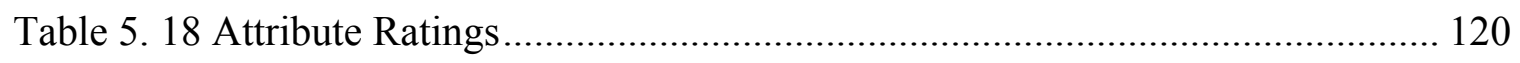

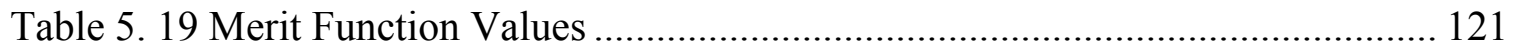

Table 6. 1 Hypotheses Verification Outline ........................................................... 131 


\section{SUMMARY}

Rapid Prototyping (RP) is the process of building three-dimensional objects, in layers, using additive manufacturing. Rapid Manufacturing (RM) is the use of RP technologies to manufacture end-use, or finished, products. At small lot sizes, such as with customized products, traditional manufacturing technologies become infeasible due to the high costs of tooling and setup. RM offers the opportunity to produce these customized products economically. Coupled with the customization opportunities afforded by RM is a certain degree of uncertainty. This uncertainty is mainly attributed to the lack of information known about what the customer's specific requirements and preferences are at the time of production. In this thesis, the author presents an overall method for selection of a RM technology, as an investment decision, under the geometric uncertainty inherent to mass customization. Specifically, the author defines the types of uncertainty inherent to RM (epistemic), proposes a method to account for this uncertainty in a selection process (interval analysis), and proposes a method to select a technology under uncertainty (Decision Theory under strict uncertainty). The author illustrates the method with examples on the selection of an RM technology to produce custom caster wheels and custom hearing aid shells.

In addition to the selection methodology, the author also develops universal build time and part cost models for the RM technologies. These models are universal in the sense that they depend explicitly on the parameters that characterize each technology and the overall part characteristics. 


\section{CHAPTER 1 CUSTOMIZATION AND RAPID MANUFACTURING}

\subsection{BACKGROUND AND MOTIVATION FOR RESEARCH}

Mass Customization (MC) can be defined as the ability to provide customized, individually designed products at low to medium production volumes at relatively low cost. As displayed in Figure 1.1, mass customization leads to high profits in low to medium production volumes, whereas mass production is advantageous in high volume production.

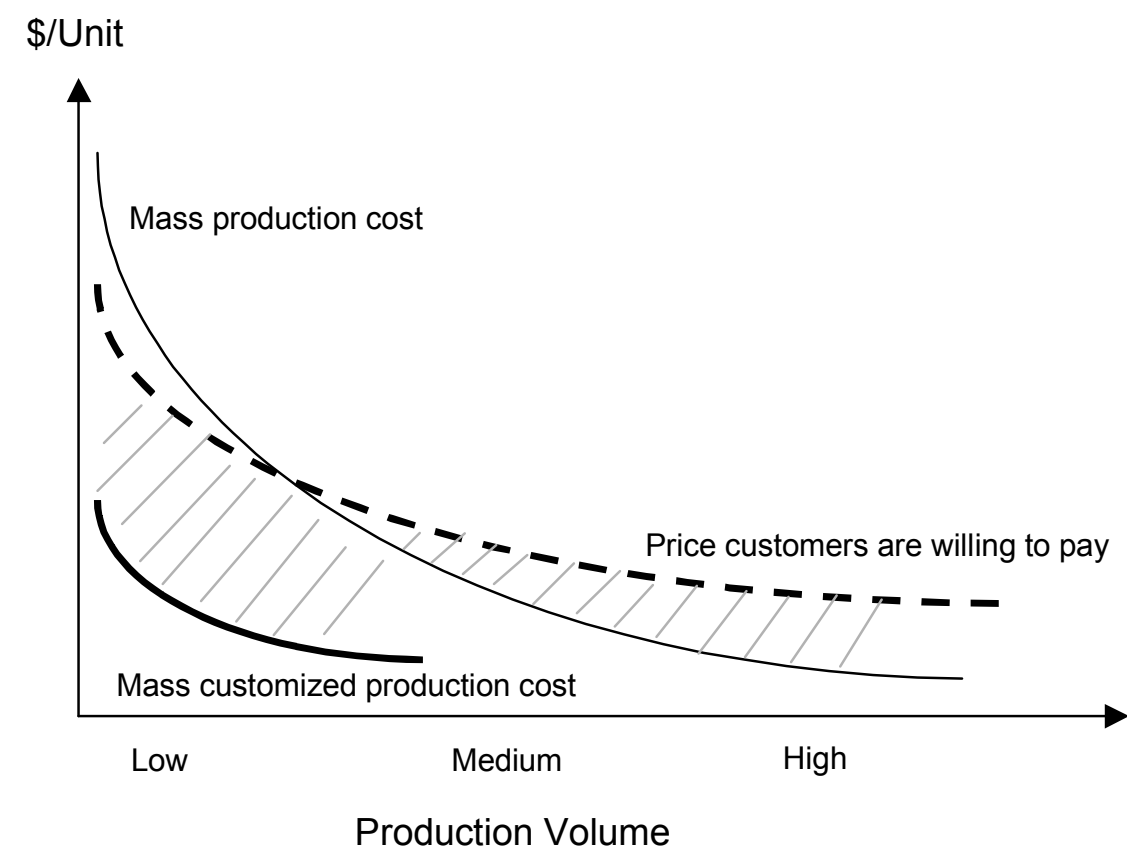

Figure 1.1 The Economic Implications of Mass Customization ${ }^{1}$

MC can be achieved through high process agility, flexibility, and integration ${ }^{2}$. Davis (1989) also argues that MC must reach customers as in the mass market economy but treat them individually as in the pre-industrial economies. There are several factors that affect the success of MC. These factors include:

- Information exchange in the dynamic translation of customer demands to product variety 
- Existence of flexible, advanced manufacturing technologies that allow customization at low cost

- Demand for product variety and customization

Information exchange is one of the main factors affecting the success of MC. The degree (level) of customization defines the volume of information needed to provide the customization. Da Silveira et al. ${ }^{3}$ defines eight generic levels of customization, which include: (1) Standardization (standardized products), (2) Usage, (3) Package and distribution, (4) Additional services, (5) Additional custom work, (6) Assembly (arranging modular components), (7) Fabrication (manufacturing of customer-tailored products following basic, predefined designs), and (8) Design (products developed according to individual customer needs). The amount and depth of information collected from the customer is determined from the degree of customization ${ }^{4}$. At the highest level of customization, Design (level 8), the geometry of the product is customized for the user. This is the type of customization that will be addressed in this thesis.

Another factor affecting the success of $\mathrm{MC}$ is the existence of flexible manufacturing systems to produce these customized parts. Rapid Prototyping (RP) is the collective name given to layer-based manufacturing technologies which build parts directly from computer models. This process is done quickly, relative to other "one-off" manufacturing techniques. In the RP process, a CAD model is developed and converted to a .STL file, which is the standard RP file format which represents the model as an "assembly of planar triangles"5. The .STL file is then sliced into thin cross-sectional layers and these layers constructed, one atop another, using the RP machine. The completed part is then clean and finished.

Companies of all sizes rely on RP in an effort to reduce time to market, improve quality, and reduce costs ${ }^{6}$. Traditionally, RP has been used only to make prototypes, as opposed to final products. Rapid Manufacturing (RM) is the use of RP technologies to manufacture end-use products, or finished parts. Recent studies have shown that companies have a strong interest in using RP to produce customized products. Some 
examples include Siemens and Phonak, which manufacture hearing aid shells, Boeing's Rocketdyne, which manufactures hundreds of parts for International Space Station and the space shuttle fleet, F-18 fighter jets, etc. ${ }^{6}$. There is also strong interest by the biomedical field in these types of technologies.

Because of their layer-by-layer construction, RP technologies have many advantages over traditional manufacturing technologies, such as injection molding, etc. In the context of RM, these advantages over include:

- Complex geometry at no extra cost Rapid Manufacturing makes it possible to manufacture complex geometry with little to no additional cost. This is not the case with conventional manufacturing technologies, where the production cost of a part is directly related to complexity of its design. The geometric complexity that RM affords can include low volume ratio structures (truss structures), as well as compliant mechanisms.

- Design Freedom RM offers complete design freedom and flexibility. Without the limitations placed on the designer by traditional manufacturing technologies, designers are able to design products with much design freedom. For example, in the design of products for injection molding, designers must account for draft angles, wall thickness, parting lines, etc. RM does not put these restrictions on the designer. The flexibility and freedom afforded by RM will directly impact the way that products are designed and developed today by eliminating the manufacturing constraints placed on the designer.

- Zero Tooling Recent trends show that customized products are becoming more in demand in the consumer marketplace. In order to be able to compete in the future, companies must be able to economically produce customized products. With conventional manufacturing technologies, tooling costs takes up a large portion of the upfront manufacturing costs. Large lot sizes are used to distribute these upfront costs amongst the parts. With customized products, and small lot sizes, this large tooling cost cannot be spread amongst thousands of parts, and therefore, makes producing custom products infeasible in many instances. RM 
offers the ability to produce large amounts of highly customized parts at a relatively fast pace.

The third main factor affecting the success of $\mathrm{MC}$ is customer demand. As mentioned earlier in this section, one of RM's main advantages is its ability to produce customized parts. Recent trends show that customized products are becoming more in demand in the consumer marketplace. As displayed in Figure 1.2, where there was once a shift from craft production (low production run, large variety offered) to mass production (large production runs, low variety offered), current market conditions show a shift towards customized products.

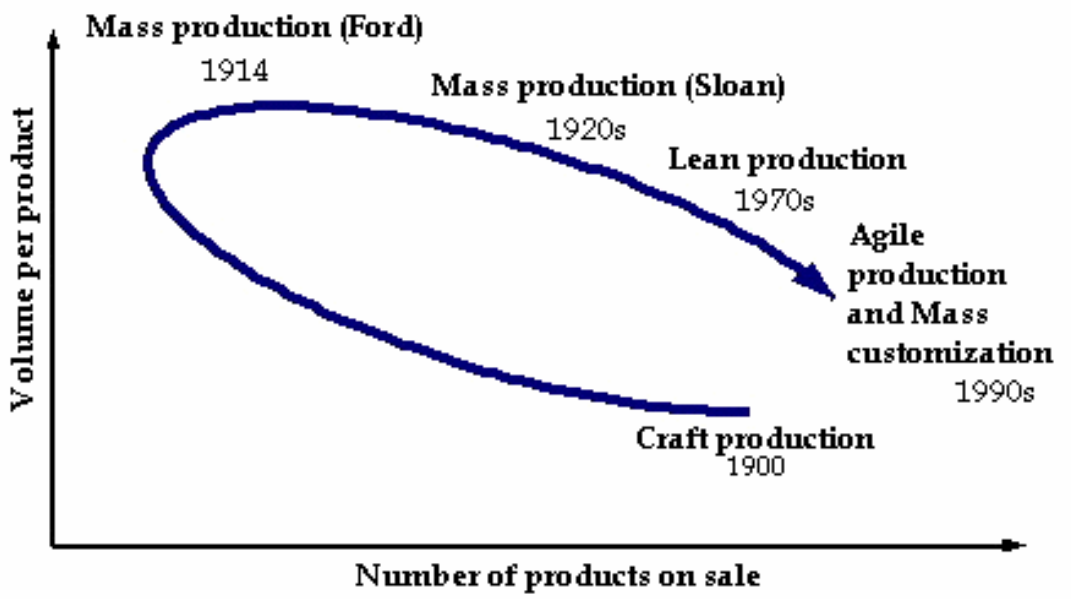

Figure 1. 2 Paradigm shifts in Manufacturing ${ }^{7}$

In order to be able to compete in the future, companies must be able to economically offer variety. At large lot sizes, conventional manufacturing technologies have proven to be the most economical. At small lot sizes (such as the case for customized parts), because of the high cost of tooling and setup, conventional manufacturing technologies become infeasible. This is where RM is key. RM offers the ability to produce large amounts of highly customized parts at a relatively fast pace. This customization ability introduces considerable amount of uncertainty about what the customer wants and will choose. In this case, the uncertainty lies in the geometric shape of the customized parts. 


\subsection{RESEARCH PROBLEM AND CURRENT APPROACHES}

Given RM's relatively recent introduction, there is still a lot of skepticism surrounding these technologies. Some particular areas of concern are the part cost, build time, and production quality of the parts produced using RM, compared to that of conventional manufacturing technologies. In other words, "how much will it cost", "how fast can we produce it", and "how good is the part"? Cost, development and manufacturing time, and production quality are all primary drivers of the current consumer marketplace.

RM introduces the ability to provide customization opportunities. The uncertainty, due to customization, involved in the RM technology selection process is mainly attributed to the lack of information about the customer's requirements and preferences. When dealing with custom manufacturing, one of the main challenges the designer will encounter is being able to account for the large amount, and varying types, of uncertainty that is introduced with customization. This will be critical in estimation of the part cost and manufacturing time of the products. Equally important is the challenge of selecting one of these technologies out of over 34 worldwide manufacturers of these RP machines.

In this thesis, we consider the selection of a RM technology for investment. Specifically, the decision problem that is considered in this thesis is as follows:

"A decision maker (DM) is attempting to select a RM technology, for investment purposes, that can be used for the production of customized products (parts)."

In considering this scoped decision problem, several key assumptions must also be noted. These assumptions are as follows:

- Geometric uncertainty is the only uncertainty considered: By making this assumption, we isolate the affects of customization on the selection problem.

- This decision is in the context of investment: This means the customer has decided to purchase a RM technology to produce these customized parts.

- True customization: Individually-designed products for customers' needs 
- Ignorance (limited knowledge): This means that we assume the decision maker has limited knowledge of what the customer will choose at the time of production. It is noted that the decision maker may have past likelihood information available. However, in this decision problem, the decision maker either has no past information or has chosen not to use this information for various reasons. These reasons may include: completely new market space, changing market conditions and customer needs, etc. This assumption is not ideal for all cases, except where information is limited.

- Defined design space: Customers are limited by the range of customization offered.

Given the decision problem described above, there have been several methods developed to account for uncertainty in the selection process, namely catalog design ${ }^{8}$, the utilitybased selection Decision Support Problem (usDSP) ${ }^{9,10}$, and the interval-based selection DSP ${ }^{11}$. These methods are discussed in further detail below.

"Catalog design is a procedure in which a system is assembled by selecting standard components from catalogs of available components" ${ }^{8}$. In this work, the authors define the fuzzy selection DSP and the Bayesian selection DSP. The fuzzy selection DSP uses fuzzy set theory (fuzzy numbers) to model the imprecision in the information. A fuzzy number is considered an uncertain parameter that is characterized by either a set of real numbers or a membership function. The Bayesian selection DSP uses Bayesian probabilities to model stochastic information. In Bayesian statistics, an uncertain parameter is represented by a probability density function (PDF), which describes the degree of belief of the uncertain parameter.

Both the fuzzy and Bayesian selection DSP formulations require that some information be assumed (whether membership or likelihood), either in the form of fuzzy sets or PDFs. In the context of selection for RM, this information cannot be assumed based on the assumption of ignorance. Another drawback of these methods (in the context of selection 
for RM) is that they are computationally expensive. This expense comes mainly when propagating the fuzzy and probabilistic information.

"Utility based selection DSP provides structure and support for using human judgement in engineering decisions involving multiple attributes and facilitates the explicit consideration of a decision maker's preferences in the context of risk and uncertainty" 9 . UsDSP is based on the combination of the constructs of utility theory and selection DSP. By complementing selection DSP with utility theory, usDSP allows the inclusion of decision maker risk preferences in the selection process, as well as a basis for making decisions under uncertainty (expected utility). Similar to the Bayesian selection DSP, usDSP also assumes that probability information is available. In usDSP, selection is based on decision maker risk preferences and uncertainty in the performance of the alternatives.

As in the case of catalog design, usDSP is also computationally expensive. This expense comes in the determination of the decision maker's risk preferences, as well as the computation of the expected utility. Propagating the uncertainty is also computationally expensive.

Interval-based selection DSP ${ }^{11}$ complements the selection DSP with interval analysis. In this method, exact interval arithmetic is used to represent the uncertainty "brought on by a lack of knowledge" 11 in the selection process. In interval-based selection DSP, selection is based on the dominance of one alternative over the others.

This method is considered computationally inexpensive, due to its use of intervals to represent uncertainty. However this method does not provide an explicit manner in which to select under uncertainty when performance is not deterministically dominant. In other words, when performance intervals overlap, how does one perform selection? 


\subsection{RESEARCH GAP, QUESTIONS, AND HYPOTHESES}

Based on the review of the current approaches for selection under uncertainty in Section 1.2 , in the context of our decision problem, the following research gap needs to be addressed:

"Currently, there are no methods for considering geometric uncertainty (due to customization) in the selection of a RM technology for investment."

Given this research gap, the focus of this research is as follows:

- Investigate selection in the context of RM technology investment

- Investigate methods for representing/propagating geometric uncertainty in the selection process.

- Develop explicit criteria for selection under geometric (epistemic) uncertainty in the context of RM.

- Develop methods for the assessment of selection attributes (such as build time and part cost for RM) under uncertainty.

To address the research gap presented above, the author sets out to answer the following primary research question of this thesis:

\section{"How can investment decisions be supported in the selection of a Rapid Manufacturing technology for customized products?"}

To answer the primary research question, it is necessary to address several, more specific, research questions. The secondary research questions are as follows:

Given customization in the context of RM, the geometric uncertainty brought about by lack of knowledge of customer preferences at the time of selection (epistemic uncertainty) is considered. Question 1 addresses how one would account for geometric uncertainty in the selection process. Answering this question addresses how the decision 
maker can represent these types of uncertainty and how this uncertainty should be propagated through the selection process.

Question 1: How can the selection DSP be extended to account for the uncertainty associated with customization in the context of Rapid Manufacturing?

Hypothesis 1: By extending the selection DSP with interval accounting and analysis, the decision maker is able to consider the uncertainty associated with customization in the selection process.

Now that the uncertainty has been propagated to the performance measures of the respective technologies, the issue turns to selecting a technology given these uncertain performance measures. By using interval analysis, the decision maker is assumed to only have information regarding the bounds of the uncertain parameter. Within Decision Theory, this type of uncertainty is termed strict uncertainty. Question 2 addresses how one is to select a technology for investment under this type of uncertainty.

Question 2: How can the selection DSP be extended to enable the designer to select a RM technology for investment under uncertainty?

Hypothesis 2: By extending selection DSP with Decision Theory under strict uncertainty, the decision maker is able to select a technology, for investment, under uncertain parameters.

Question 3 deals with the selection criteria, or attributes, used in the selection process. This question addresses the 'how much' and 'how fast' questions that are inherent to these budding technologies. The central issue involved in this question is the lack of support when it comes to answering these 'how much' and 'how fast' questions. In academia and industry, this issue has not been thoroughly addressed. Although there are 
several build time and cost estimators specifically linked to many of the RM process, all require build files or CAD models for solution. When dealing with a range of products, due to customization, explicit CAD information may not be available. With this lack of explicit information, how does one characterize the performance of these machines? Question 3 addresses this issue.

\section{Question 3: How can part cost and build time be quantified for Rapid Manufacturing technologies with limited geometric information due to customization?}

Hypothesis 3: Parametric build time and part cost models can be developed that depend explicitly on the parameters that characterize each technology and the overall part characteristics.

The above research questions will be addressed throughout this thesis. The hypotheses will be verified according to the plan put forth in Section 1.5.

\subsection{ORGANIZATION OF THIS THESIS}

In Chapter 2, the author will lay the theoretical foundation to support Selection for Rapid Manufacturing. The three foundational constructs are the selection DSP (Section 2.1), uncertainty handling (Section 2.2), and selection under epistemic uncertainty (Section 2.3. The author will also review the literature that supports these constructs in Chapter 2 .

In Chapter 3, the author will synthesize these foundational constructs and introduce the Selection for Rapid Manufacturing methodology. In this chapter, the author addresses the sources of uncertainty in the RM process (section 3.1), as well as introduces the method proposed to account for these uncertainties in the selection problem. The author also details the Selection for Rapid Manufacturing under epistemic uncertainty methodology, including the word formulation and steps for implementation. 
In Chapter 4, the author introduces the part cost and build time estimation models used in the selection method proposed for RM. These are the two selection attributes that are most affected by the geometric uncertainty due to customization. The author also introduces the Matlab GUI tool that was developed for build time and part cost estimation.

In Chapter 5, the author provides two illustrative examples for selection of RM technologies. Both examples address the uncertainty that is introduced with customization of products. The first example considers the direct production of caster wheels, and the second example considers the production of custom hearing aid shells. The method will be compared against the results from a selection process where uncertainty is not considered.

In Chapter 6, the research questions and their respective hypothesis are revisited. The specific contributions to the body of knowledge on RM are also reviewed in this chapter.

\subsection{THE VALIDATION AND VERIFICATION STRATEGY FOR THIS THESIS}

The validation and verification strategy in this thesis is two fold. The first strategy addresses the verification of the hypotheses proposed to answer the secondary research questions proposed in Section 1.2. The second strategy involves the validation of the extended selection DSP proposed in this thesis, which is referred to as Selection for Rapid Manufacturing.

\subsubsection{Verification of Hypotheses}

In this thesis, three hypotheses are proposed to address the secondary research questions in Section 1.2. There are four ways in which these hypotheses will be verified: through the theoretical model of the selection method, the mathematical models for build time and part cost, and two illustrative examples of selection. The first example proceeds through selection for a RM technology for the production of custom caster wheels and the second for production of custom hearing aid shells. With these examples, we apply selection for 
$\mathrm{RM}$ in real world scenarios, thus giving us a good assessment of the usefulness of the method.

The hypotheses have been divided into factors that will be tested using the three methods above. For Hypothesis 1, the author will test 1) that the selection DSP can be extended to include epistemic uncertainty and 2) the ability for uncertainty to be propagated in the selection problem using interval arithmetic. In Hypothesis 2, the author tests that 3 ) the selection DSP can be extended with use of decision theory selection criterion for selection under uncertainty. In Hypothesis 3, the author tests that 4) build time can be quantified with limited geometric information and 5) that part cost can be quantified with limited geometric information.

A summary of the test factors for the hypothesis and how they will be verified is displayed in Table 1.1.

Table 1. 1 Hypotheses Verification Outline

\begin{tabular}{|c|c|c|c|c|c|}
\hline Test Methods & 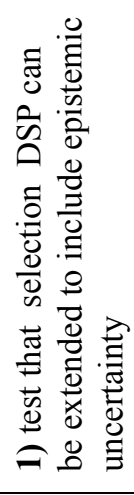 & 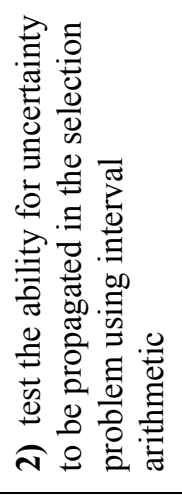 & 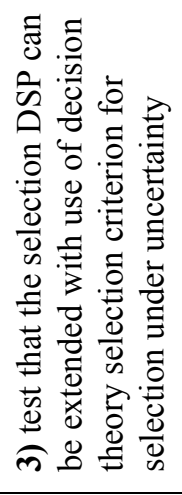 & 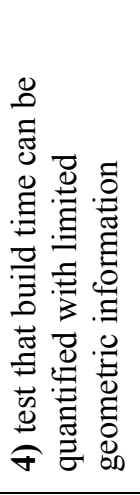 & 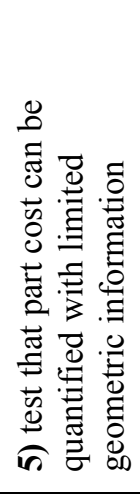 \\
\hline $\begin{array}{l}\text { Selection for RM } \\
\text { Theoretical Model } \\
\text { (Chapters } 2 \text { and 3) }\end{array}$ & $\mathbf{X}$ & $\mathbf{X}$ & $\mathbf{X}$ & & \\
\hline $\begin{array}{l}\text { Build Time and Cost } \\
\text { Model (mathematical } \\
\text { models) (Chapter 4) }\end{array}$ & & & & $\mathbf{X}$ & $\mathbf{X}$ \\
\hline $\begin{array}{l}\text { Example 1: Direct } \\
\text { production of custom, steel } \\
\text { caster wheels } \\
\text { (Chapter 5) }\end{array}$ & $\mathbf{X}$ & $\mathbf{X}$ & $\mathbf{X}$ & & $\mathbf{X}$ \\
\hline $\begin{array}{l}\text { Example 2: Direct } \\
\text { production of custom } \\
\text { hearing aid shells } \\
\text { (Chapter 5) }\end{array}$ & $\mathbf{X}$ & $\mathbf{X}$ & $\mathbf{X}$ & $\mathbf{X}$ & $\mathbf{X}$ \\
\hline
\end{tabular}


Since the focus of this thesis (and the research questions) is the extension of the selection DSP for RM, the verification of the hypotheses also involves the validation of the extended selection method. This strategy is presented in Section 1.5.2.

\subsubsection{Validation of Selection for Rapid Manufacturing}

The validation square proposed by Pederson et al. ${ }^{12}$ is used for validation of the selection method proposed in this thesis, Selection for Rapid Manufacturing. Pederson et al. believe that validation in engineering design, because it is based largely on designers' subjectivity, "cannot be pursued in formal, rigorous, quantitative verification based on

logical induction and/or deduction"13. Pederson et al. ${ }^{12}$ have noted that "knowledge validation becomes a process of building confidence in its usefulness with respect to a purpose."

The framework presented by Pederson et al. is presented in Figure 1.3 and 1.4 (close-up of validation square). As seen in the figure, there are 4 aspects to the Validation Square:

(1) Theoretical Structural Validation, (2) Empirical Structural Validation, (3) Empirical Performance Validation, and (4) Theoretical Performance Validation. 


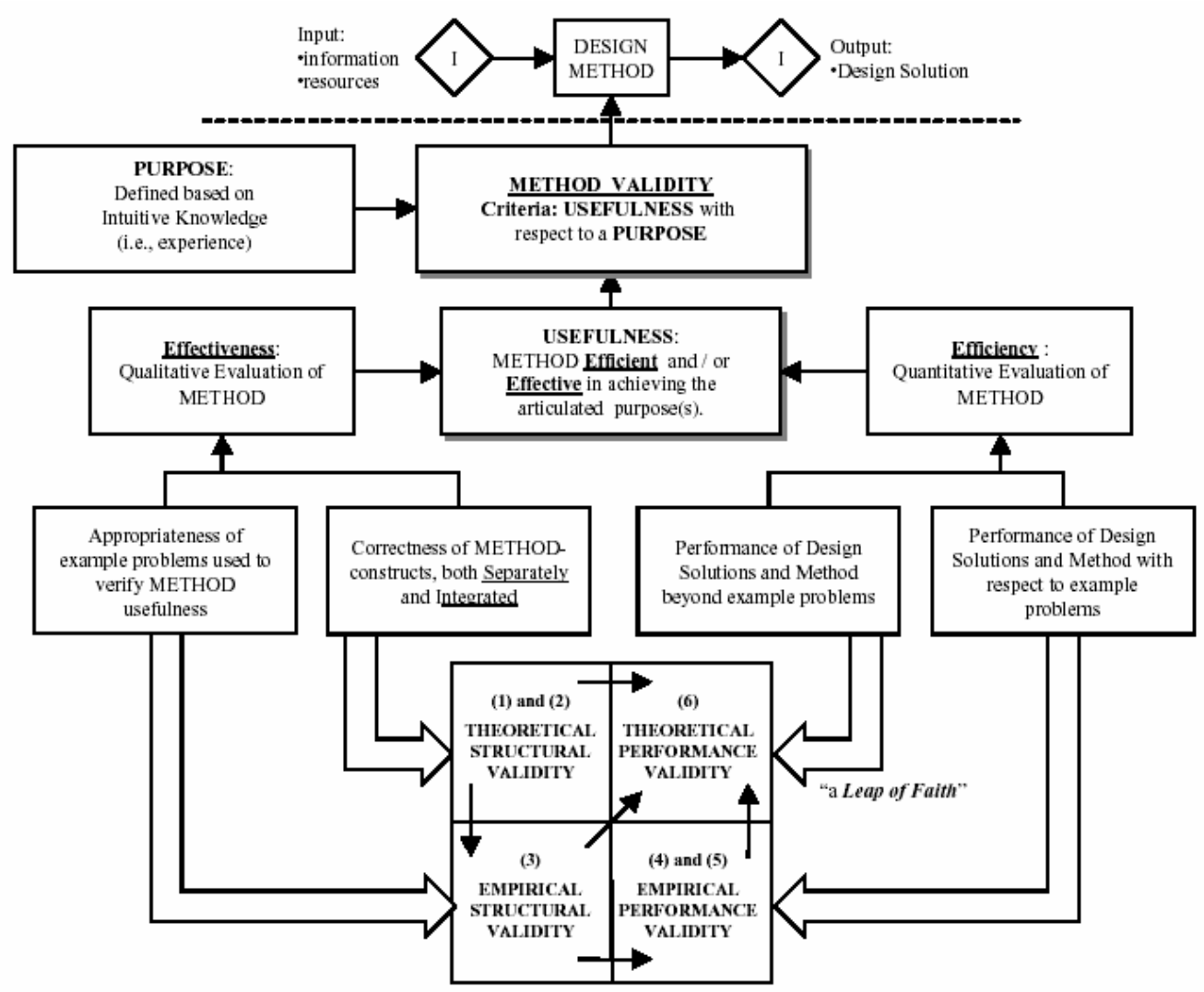

Figure 1. 3 Design Method Validation: A Process of Building Confidence in Usefulness ${ }^{12}$

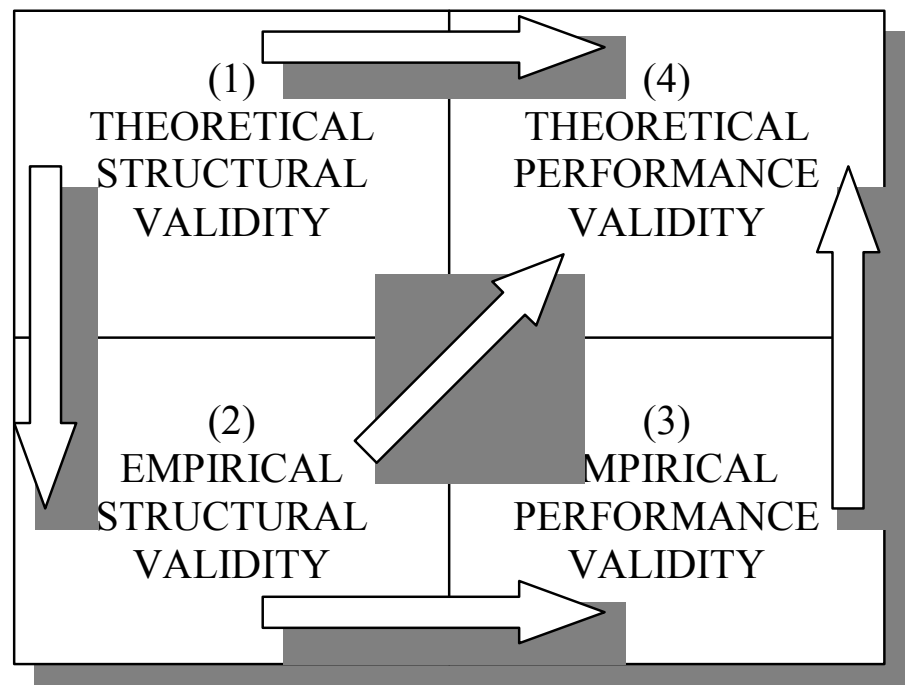

Figure 1. 4 Validation Square Closeup ${ }^{12}$ 
Theoretical Structural Validation (TSV) involves checking the individual constructs and assumptions upon which the method is built, as well as checking the internal consistency of the method when combining the individual constructs. Usually, this involves searching and referencing the relevant literature, as well as evaluation of the individual constructs and method as a whole.

In this thesis, the TSV is evaluated in Chapters 2, 3, and 4. In Chapter 2, each individual construct of Selection for RM is critically reviewed. In Chapter 3, the method as a whole is presented and its internal consistency evaluated. The build time and part cost estimation models used within Selection for RM are critically reviewed in Chapter 4.

Empirical Structural Validation (ESV) is sometimes regarded as a measure of the method's appropriateness. ESV is accomplished by showing that the example problems used are appropriate for the method proposed. Also, the data used in the example problem should be able to be used to support conclusions drawn.

In this thesis, ESV is presented in Chapter 5. In Chapter 5, two examples are presented: selection for the direct production of caster wheels and for the production of custom hearing aid shells.

Empirical Performance Validation (EPV) is the evaluation of the 'usefulness' of the proposed method. EPV is accomplished by using example problems in such a way that the conclusions drawn from the example can be used to evaluate the proposed method. Also, it is important to show that the results obtained from the example problems are because of the proposed method and not because of chance.

In this thesis, EPV is demonstrated in Chapter 5. In Chapter 5, the usefulness of Selection for RM under Uncertainty is evaluated by comparing the results against a selection method where uncertainty is not considered (Selection DSP). Since build time and part cost are big factors in the selection problem presented in this thesis, the performance of the models is also evaluated in Chapter 4. 
Theoretical Performance Validation (TPV) involves building confidence in the ability to extend the proposed method beyond the scope of the example problem to a general class of problems. TPV involves establishing the general usefulness of the proposed method. TPV is demonstrated in Chapter 6 of this thesis by illustrating the extensiveness and relevance of the proposed method beyond the scope of the illustrative examples.

The validation strategy suggested above has been outlined in Table 1.2.

Table 1. 2 Validation Strategy Outline

\begin{tabular}{|l|c|c|c|c|c|}
\hline & Ch. 2 & Ch. 3 & Ch. 4 & Ch. 5 & Ch. 6 \\
\hline Theoretical Structural Validity (TSV) & $\mathrm{X}$ & $\mathrm{X}$ & $\mathrm{X}$ & & \\
\hline Empirical Structural Validity (ESV) & & & $\mathrm{X}$ & $\mathrm{X}$ & \\
\hline Empirical Performance Validity (EPV) & & & & $\mathrm{X}$ & \\
\hline Theoretical Performance Validity (TPV) & & & & & $\mathrm{X}$ \\
\hline
\end{tabular}

In Chapter 1, the research questions and validation strategy for this thesis was presented. In Chapter 2, the author will lay the theoretical foundation to support Selection for Rapid Manufacturing. 


\section{CHAPTER 2 THEORETICAL FOUNDATION: ELEMENTS OF SELECTION FOR RAPID MANUFACTURING}

\subsection{THE SELECTION DECISION SUPPORT PROBLEM}

Engineering Design can be seen as a series of decisions that involve the selection and/or

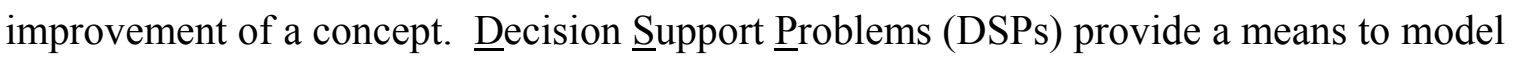
these decisions and support the decision maker in making these decisions. There are two main types of DSP available for Engineering Design: selection and compromise. The selection DSP facilitates the selection of the most feasible design alternative from a set of alternatives ${ }^{14}$. The compromise DSP facilitates the improvement of a design alternative through modification ${ }^{15}$. These DSPs are described in terms of complementary word and mathematical formulations. Due to the scope of this thesis, the selection DSP is pursued. The selection DSP is discussed in detail in the following sections.

\subsubsection{Description, Word Formulation, and Mathematical Formulation}

In this section, a detailed description of the selection method proposed in this thesis is presented. The solution of the selection DSP involves identifying the set of feasible design alternatives, the principle attributes (criteria) influencing selection, and the relative importances of the attributes. The alternatives are then rated with respect to each attribute, and a merit function value determined for each attribute. The alternatives are then ranked based on these merit function values, with higher merit functions indicating preference.

The word formulation for the selection DSP ${ }^{16}$ is presented in Figure 2.1. 
Given A set of feasible alternatives.

Identify The principle attributes influencing selection.

The relative importance of the attributes.

Rate The alternatives with respect to each attribute.

Rank The feasible alternatives in order of preference based on the attributes and their relative importance

Figure 2. 1 The Selection Decision Support Problem ${ }^{16}$

A summary of the steps involved in its implementation are presented in Figure 2.2.

\section{Steps for the Selection Decision Support Problem}

Step 1 Describe the alternatives and provide acronyms

Step 2 Describe each attribute, specify its relative importance and provide acronyms

Step 3 Specify scales, rate the alternatives with respect to each attribute.

Step 4 Normalize the attribute ratings

Step 5 Evaluate the merit function for each alternative

Step 6 Post-Solution Analysis and Verification of results

Figure 2. 2 Summary of Steps for selection Decision Support Problem ${ }^{14}$

A detailed description of the 6 steps of the selection DSP are displayed in Figure 2.2 is presented next ${ }^{14}$.

\section{Step 1. Describe the alternatives and provide acronyms}

Describe each alternative in words, including its advantages and disadvantages, and provide meaningful acronyms for each. If possible, provide illustrations of the alternatives.

\section{Step 2 Describe each attribute, specify its relative importance and provide acronyms}

The next step in solving the Selection DSP is to identify the attributes by which the alternatives will be evaluated. Depending on the demands of each problem, the attributes 
will vary. All relevant attributes should be included. The description of each attribute should be comprehensive and understandable. Also, one should provide meaningful acronyms from the attributes.

In order to specify the relative importance of the attributes, a pair-wise comparison is used. It is noted that other methods, such as the ranking method or arbitrary selection, can be used. In the pair-wise comparison method, each of the attributes is rated as better than, worse than, or equal to each of the other attributes. For the comparison, a value of 1 is given to the attribute that is better, whereas a 0 is given to the other attribute. If the attributes are considered equal, both attributes receive a value of zero. Next, the values for each attribute are summed and normalized to ensure the relative importances sum to one. To prevent an attribute receiving a total value of zero, a dummy attribute is introduced. In this comparison, the attribute is always preferred to the dummy.

\section{Step 3 Specify scales, rate the alternatives with respect to each attribute.}

There are four main types of scales: ratio, interval, ordinal, and composite. The type of information available determines the type of scale chosen. The ratio scale is used when quantitative, physically meaningful units are available for an attribute. When an attribute can only be qualified in words, use the ordinal scale. The interval scale is used to convert the words from an ordinal scale to numerical intervals. The composite scale is used when the value of attribute is the result of computations, such as relative importance analysis.

Once the scale is chosen, the alternatives are also rated with respect to each attribute.

\section{Step 4 Normalize the attribute ratings}

The attribute ratings, from Step 3, are on nonuniform scales. Therefore, these values need to be converted to a uniform scale, or normalized. When higher values of an attribute rating are preferred, the following equation should be used to normalize the attribute ratings ${ }^{16}$ :

$$
N R_{i j}=\frac{A_{i j}-A_{j, \min }}{A_{j, \max }-A_{j, \min }}
$$


When lower values are preferred, the following equation should be used to normalize the attribute ratings ${ }^{16}$ :

$$
N R_{i j}=\frac{A_{j, \text { max }}-A_{i j}}{A_{j, \max }-A_{j, \min }}
$$

where $A_{i j}$ is the attribute rating w.r.t alternative $j, A_{j, \max }$ is the maximum value of attribute $i$, and $A_{j, \min }$ is the minimum value of attribute $i$, and $N R_{i j}$ is the normalized rating of the attribute $i$ with respect to alternative $j$.

\section{Step 5 Evaluate the merit function for each alternative}

The merit function values of the alternatives are calculated using the following equation:

$$
M F_{j}=\sum_{j=1} I_{i} \cdot N R_{i j}
$$

where $M F_{j}$ is the merit function of alternative $j, I_{i}$ is the relative importance of attribute $i$, and and $N R_{i j}$ is the normalized rating of the attribute $i$ with respect to alternative $\mathrm{j}$.

\section{Step 6 Post Solution Analysis and Verification of results}

In this step, the results from Step 5 are reviewed and verified. The designer must determine if the results seem logical and reasonable. Verification may involve changing the weighting schemes (relative importances) of the attributes for different scenarios. Once the merit functions are recalculated, the alternative rankings should be compared and evaluated.

\subsubsection{Critical Review of Selection DSP}

As stated earlier, the selection DSP is best suited for situations when the designer is choosing a feasible alternative from a set of alternatives. This is a proven method and has been applied in various contexts, including catalogue design ${ }^{8}$ and design of frigates 16, to name a few. "The main advantages of the selection DSP are its provision of context, structure, and domain independence" ${ }^{10}$. Another advantage is that this 
methodology can be used at all stages in the design process. When information is limited (qualitative), the preliminary selection DSP and when information is quantitative, the selection DSP can be used. In Step 6 of the selection DSP, post-solution sensitivity analysis, helps to ensure the result's robustness of the solutions with respect to the relative importances of the respective attributes. The robustness of solution attained from the sensitivity analysis is also considered advantageous and unique to the selection DSP.

Although the selection DSP has many advantages, it does incur certain limitations when applied in specific domains like RM. The selection DSP offers no explicit way of dealing with uncertainty in the process. Specifically, when the merit function values are uncertain, how does one select an alternative when one does not clearly dominate another? Also, the selection DSP does not account for the decision maker's attitudes towards risk in the selection process. In situations of uncertainty, it is valuable to include the decision maker's risk preferences in the decision process. This assures that the solution of the selection problem is consistent with the intention and beliefs of the decision maker. Due to these limitations, in this thesis, the selection DSP will be extended to handle uncertainty and account for the decision maker's risk preferences, specifically in the context of RM. These suggested extensions will be addressed in Sections 2.2 and 2.3 .

\subsection{UNCERTAINTY REPRESENTATIONS}

Uncertainty in the design of mechanical systems is unavoidable. A design process can be seen as the method for systematically reducing the uncertainty associated with a design. Uncertainty can be divided into 2 distinct types: aleatory and epistemic. Aleatory uncertainty can be considered as irreducible or inherent uncertainty ${ }^{17}$, due to variability 18. Epistemic uncertainty "is a potential deficiency in selecting the best action in a decision due to lack of knowledge" ${ }^{18}$. Epistemic uncertainty can be reduced by collecting additional information or acquiring additional knowledge ${ }^{18}$. Aleatory uncertainty can be easily quantified (through experimentation) and represented by a probability density function (PDF), while epistemic uncertainty is predictive in nature, 
thus lacking the information for representation with a complete PDF. This is mainly due to the fact that with a PDF, you are predicting the likelihood of an event to occur. With epistemic uncertainty, this likelihood cannot be quantified due to a lack of information or data. Aleatory and epistemic uncertainty are further discussed in the following sections.

\subsubsection{Aleatory Uncertainty and its Representations}

The most common, and appropriate, method for representing aleatory uncertainty in engineering design is with probabilities. Probability theory provides the mathematical structure traditionally used to represent uncertainty and is based on assigning probabilities to events that may occur. These probabilities represent the 'likelihood' of an event to occur. "With complete and sufficient information, aleatory uncertainty is well represented by a probabilistic function, such as a PDF" ${ }^{19}$.

Probability theory is built on 3 axioms which are displayed in Figure 2.3.

Give a sample space $(S)$ and a probability function, $p(A)$, associated with each event $A$

(P1) $p(A) \geq 0$ for each event $A$

(P2) $p(S)=1$

(P3) If there exist a countable set of events $\left\{A_{1}, \ldots ., A_{\mathrm{n}}\right\}$, and if these events are mutually exclusive, then

$$
p\left(A_{1} \cup \ldots . \cup A_{n}\right)=p\left(A_{1}\right)+\ldots . .+p\left(A_{n}\right)
$$

Figure 2. 3 Three axioms of Probability Theory ${ }^{20}$

\subsubsection{Epistemic Uncertainty and its Representations}

There are several formal methods for modeling epistemic uncertainty in engineering design: probability theory, possibility theory ${ }^{21}$, evidence theory ${ }^{22}$, and interval analysis 23. The main differentiating factor between these theories is the manner in which they represent the likelihood of an event to occur. Of all the formal methods for representing epistemic uncertainty, interval analysis is the only method that does not assume a 
likelihood, or membership, distribution to the events. These above theories are discussed briefly below.

\subsubsection{Probability Theory}

In the probabilistic approach of representing epistemic uncertainty, under Laplace's Principle of Insufficient Reason, uncertainty is modeled with a uniform distribution across the range. Laplace ${ }^{24}$ argued that 'knowing nothing at all about the true state of nature' is equivalent to 'all states having equal probability' ${ }^{25}$. Laplace's Principle of Insufficient Reason states that if a PDF was not assigned by the decision maker, then there must have been insufficient reason for the decision maker to indicate that one was more or less likely to occur than any other state. As a consequence, all states must be equally likely, or probable. Since all states are assumed equally likely, a uniform PDF can be assigned across the range.

\subsubsection{Possibility Theory}

Possibility Theory was developed based on the concept of fuzzy sets ${ }^{26}$ and is commonly used to represent epistemic uncertainty. Fuzzy sets were developed to deal with problems involving vagueness and imprecision of information. A fuzzy set $(F)$ is characterized by a membership function, $\mu_{F}$, which is used to define the degree to which

each object is a member of $F^{27}$. An event may be either a member or non-member of the set based on the membership function. In possibility theory, the membership of a fuzzy variable is given by a continuous, possibility function, $\Pi$, which can be compared to that of a PDF.

The three axioms upon which possibility theory is built are displayed in Figure 2.4. 
Given a finite set $(S)$ and a function, $\Pi$, that maps the subsets of $S$ onto a real number interval $(0,1)$

(S1) $\Pi(\phi)=0$

(S2) $\Pi(S)=1$

(S3) For every positive integer $n$ and every collection $\left\{A_{1}, \ldots ., A_{\mathrm{n}}\right\}$ of subsets of $S$,

$\Pi\left(A_{1} \cup \ldots . . \cup A_{n}\right)=\max \left\{\Pi\left(A_{1}\right), \ldots . ., \Pi\left(A_{n}\right)\right\}$

then $\Pi$ is called a possibility function over $S$.

Figure 2. 4 Three axioms of Possibility Theory ${ }^{20}$

Possibility theory can be viewed as equivalent to a relaxation of axiom P3 from probability theory. Instead of additivity, possibility theory applies a weaker operation to the disjunction of multiple events ${ }^{20}$

\subsubsection{Evidence Theory}

In many practical engineering cases, both aleatory (variability) and epistemic uncertainties exist simultaneously and must, therefore, be accounted for. "Evidence theory is a generalization of classical probability and possibility theories from the perspective of bodies of evidence and their measures, even though the methodologies for manipulation of evidence are totally different. Hence, evidence theory can handle not only epistemic uncertainty, but also aleatory uncertainty in its framework"19.

Evidence theory was first developed by Dempster ${ }^{28}$, and later extended and refined by Schafer ${ }^{22}$. In evidence theory, consider any finite set $(S)$ and let $2^{S}$ denote the set of all subsets of $S$. In evidence theory, two functions are defined: belief, $\operatorname{Bel}(A)$, and plausibility, $\operatorname{Pl}(A)$. The plausibility function, $P l(A)$, is used to reflect the knowledge gained from the evidence that does not support $\bar{A}^{20}$, i.e.

$$
\operatorname{Pl}(A)=1-\operatorname{Bel}(\bar{A})
$$

The $\operatorname{Bel}(A)$ can be considered the minimum uncertainty bound of $\mathrm{A}$ and $\operatorname{Pl}(A)$ can be considered the maximum uncertainty bound of $\mathrm{A}$, where uncertainty about A can be 
represented as $[\operatorname{Bel}(A), P l(A)]$. This is also known as the belief interval, which provides a measurement of imprecision about the uncertainty value ${ }^{29}$. In the case of probability theory, uncertainty is measured by a single value for probability. See ${ }^{19,22,28-30}$ for further reference on evidence theory and how epistemic uncertainty is represented using this theory.

\subsubsection{Interval Analysis}

As stated before, interval analysis is the only method that does not assume a distribution for an event. When using the interval representation of epistemic uncertainty, uncertainty is modeled with a closed interval bounded by $z_{\min }$ and $z_{\max }$ (i.e., $Z \in\left[z_{\min }, z_{\max }\right]$ ). When epistemic uncertainty is modeled using interval numbers, the design equations are converted to intervals ${ }^{31}$. These intervals are then propagated using interval arithmetic. This process results in a bounded interval that represents the uncertainty in the results. It should be noted that interval operations must be carried through all computations to ensure the results accurately reflect the uncertainty in the results.

It should be recognized that if any parameter is uncertain, this uncertainty must be propagated through all the affected calculations. In the case of RM, the geometric characteristics of the part are uncertain, therefore the selection attributes which are affected by the geometric characteristics, mainly the build time and part cost, will also be uncertain. This propagation of uncertainty can be performed using interval arithmetic. The arithmetic of interval analysis is discussed in detail in Moore ${ }^{32}$. Selected rules for interval arithmetic are presented in Figure 2.5. 


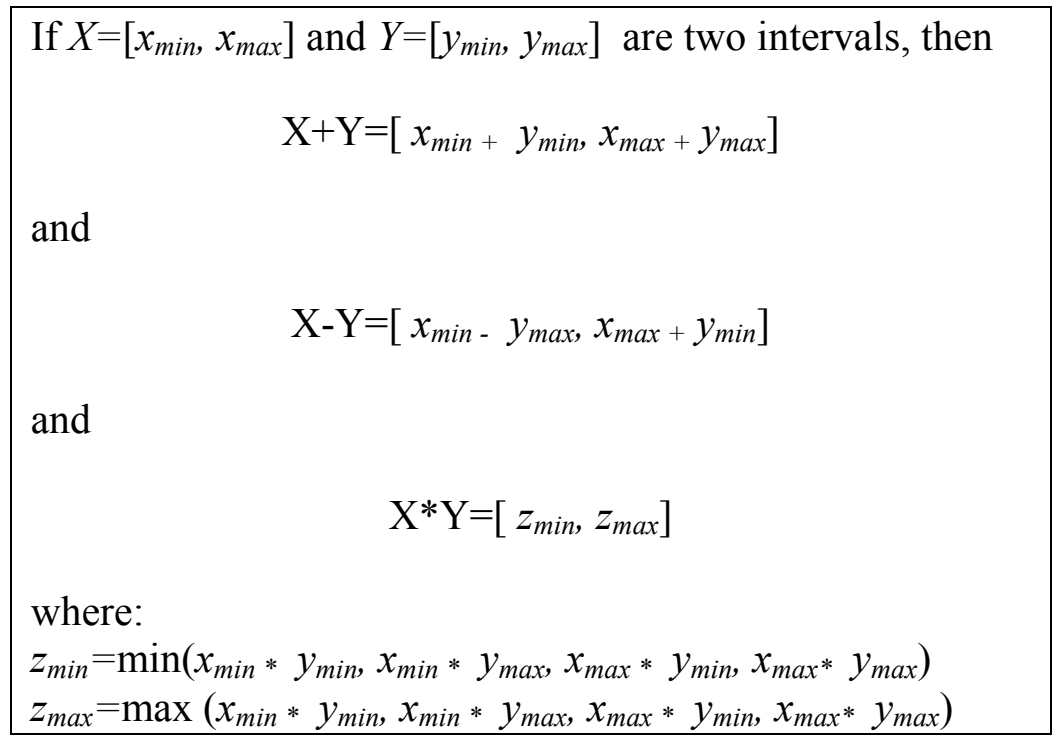

Figure 2. 5 Selected rules of interval arithmetic ${ }^{32}$

\subsubsection{Uncertainty in Engineering Systems}

Some examples of aleatory uncertainty (variability) found in engineering systems include: material properties, material characteristics, machine characteristics, etc. These types of uncertainty are considered inherently variable within the system being described. Because they are inherently variable, these types of uncertainty will always be present in engineering systems and cannot be reduced. Epistemic uncertainty, on the other hand, represents uncertainty which can be controlled and reduced. Because epistemic uncertainty stems from a lack of knowledge, with the gathering of additional knowledge, this uncertainty can be reduced.

To examine the difference between aleatory and epistemic uncertainty, consider the rapid manufacturing of custom hearing aid shells using Stereolithography (assumed random). In this example, the engineer is interested in testing the material properties of the resin, specifically, the flexural modulus of the hearing aid shell. Suppose the engineer is supplied with an infinite amount of samples in which he/she can characterize the flexural modulus with a normal probability distribution. The uncertainty in this case is considered aleatoric, since the engineer is as close to complete knowledge as possible. Due to the inherent randomness of the manufacturing process, the uncertainty cannot be further reduced. Now suppose the engineer is only supplied with 10 samples of the hearing aid 
shells. At present state, the engineer cannot accurately represent the flexural modulus with any probability distribution. In this case, the uncertainty is considered epistemic since with additional samples, the uncertainty can be reduced and further characterized.

\subsubsection{Critical Review of Uncertainty Handling Representations (Interval Analysis vs. Probability Theory)}

Although there are several formal methods for representing epistemic uncertainty (see Section 2.2.2), two commonly-used methods are probability theory and interval analysis. The choice of representation will influence the outcome of uncertainty propagation and solution. The support of the probability distributions (range of values with nonzero probabilities in a PDF) is identical to the result from interval analysis ${ }^{33}$ after the uncertainty is propagated using the two methods. The main difference is that the solution from the probabilistic approach will contain a certain density function over the range. This function defines the likelihood of an event (number) in the solution to occur.

There have been many arguments in favor of and against the use of interval analysis and probability theory when epistemic uncertainty is present. Ferson et al. ${ }^{33}$ argues that "using classical probability theory to estimate even the simple product of two uncertain parameters requires several assumptions, without which no answer could be obtained." They also go on to argue that "unless there is specific empirical information or theoretical argument to justify such assumptions, the results they produce could never be scientifically defensible." Laplace's principle assumes that if the decision maker has not assigned a PDF, then it was because there was insufficient reason for the decision maker to indicate that one state was more or less likely to occur than any other state. This argument does not consider the decision maker that has not assigned a PDF due to a lack of information (or any other reason).

Regan et al. ${ }^{34}$ argue that one of the downfalls to using intervals is that they are not able to represent all the available information about an uncertain parameter. They also argue that "intervals are only appropriate for numerical uncertainty." In our case, where there 
is limited information known about the geometric uncertainty involved, the author believes the use of the interval representation of epistemic uncertainty is justified. 


\subsection{SELECTION UNDER EPISTEMIC UNCERTAINTY}

When using Selection DSP, a Merit Function (MF) value is used as a performance measure for each alternative. If this merit function value for each alternative is certain (scalar), then selecting between alternatives is simple. For example, if $M F(B)<M F(A)$, then alternative $A$ should be selected over alternative $B$. When uncertainty (epistemic in our case) is included in the Selection DSP, these MFs will be in the form of intervals, where $M F \in\left[M F_{\min }, M F_{\max }\right]$. Comparing these intervals is not as definitive as comparing the scalar values discussed above. When comparing intervals, dominance cannot be easily determined in all cases. Table 2.1 displays two possible scenarios.

Table 2. 1 Possible scenarios of interval relations

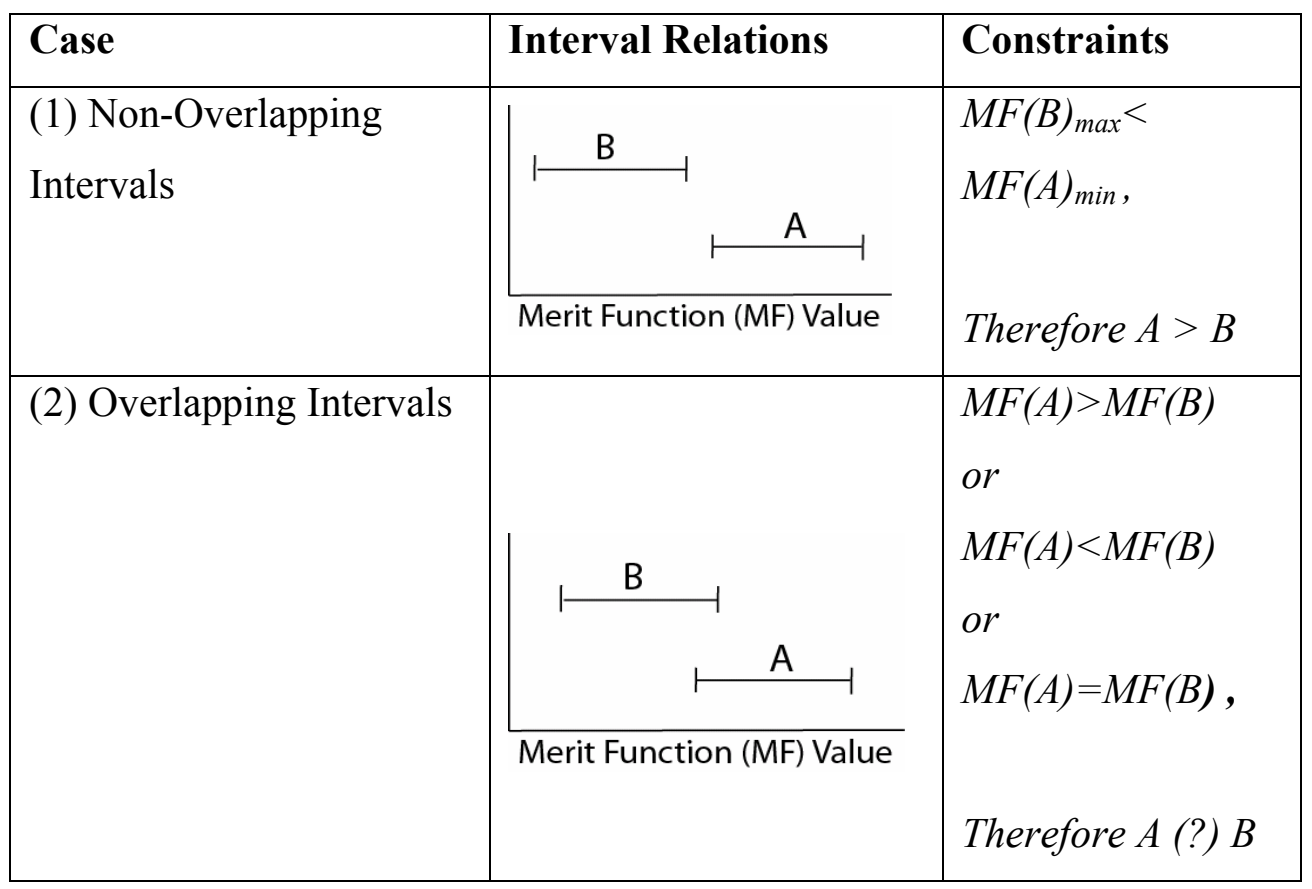

If the MF values do not overlap (Case 1), dominance can be easily determined. In that case, since $M F(B)_{\max }<M F(A)_{\min }, M F(A)$ will always be greater than $M F(B)$. Therefore, alternative A dominates alternative $\mathrm{B}$, and $\mathrm{A}$ should be selected as the best alternative. In the case of overlapping intervals (Case 2), an interval's dominance cannot be easily established unless additional information is introduced. As seen in Table 2.2, when the 
intervals overlap, depending on where the $\mathrm{MF}(\mathrm{A})$ and $\mathrm{MF}(\mathrm{B})$ actually fall within their respective intervals, $M F(A)>M F(B), M F(A)<M F(B)$, or $M F(A)=M F(B)$. As explained earlier, this determines dominance of one alternative over another. This explains the difficulty in selecting alternatives under uncertainty and the need for generalized, formal methods for selection under uncertainty. Given the presence of uncertainty, the author believes Decision Theory can be leveraged as a guide for comparing alternatives under uncertainty.

\subsubsection{Decision Theory}

In Decision Theory, decisions can be categorized according to the decision maker's knowledge about an event. These categories are as follows:

Decisions under certainty: Under certainty, the decision maker is assumed to have complete knowledge about the state of nature before the decision is made.

Decisions with risk: Under risk, "the decision maker does not know the true state of nature for certain, he can quantify his uncertainty through probability distribution” 25 .

Decisions under strict uncertainty: Under strict uncertainty, "the decision maker feels that he can say nothing at all about the true state of nature" ${ }^{25}$. Under strict uncertainty, the decision maker cannot quantify his uncertainty in any way. In other words, no probabilities can be assigned to the states of nature.

In the interval representation of epistemic uncertainty (proposed in section 2.2.4), the decision will be classified as a decision under strict uncertainty. In this case, it is assumed that there is limited knowledge about what will happen at any given instant, therefore ignorance about the likelihood of the state is assumed. Based on the assumption of strict uncertainty, the four selection criteria for decision making under strict uncertainty are described in section 2.3.2. 


\subsubsection{Four Selection Criteria for Strict Uncertainty}

Within Decision Theory, decision tables are commonly used to represent decision problems. A general form of the decision table is displayed in Table 1, where $S$ represents the set of mutually exclusive states of nature, $A$ represents the selection alternatives, and $p_{m, n}$ represents the performance of alternative $A_{m}$ at state $S_{n .}$.

\section{Table 2. 2 General decision table}

\begin{tabular}{c|ccc} 
& $S_{1}$ & $\cdots$ & $S_{n}$ \\
\hline$A_{1}$ & $p_{11}$ & $\cdots$ & $p_{1 n}$ \\
$\vdots$ & $\vdots$ & $\ddots$ & $\vdots$ \\
$A_{m}$ & $p_{m 1}$ & $\cdots$ & $p_{m n}$
\end{tabular}

There are several criteria which can be used for selection under strict uncertainty in Decision Theory. These criteria are based on the decision-maker's attitude towards risk. The main selection criteria include:

- Maximin Criterion

- Maximax Criterion

- Hurwicz Criterion

- Laplace Criterion

The criterion are discussed in detail in the following sections.

\subsubsection{Maximin Criterion}

The Maximin criterion, also called Wald's criterion ${ }^{35}$, is based on the assumption of a pessimistic decision-maker. The pessimistic decision maker is willing to forgo attractive rewards in order to definitely avoid losses. The general philosophy is to assume that the worst possible outcome will happen. With this criterion, each alternative is represented by and selected based on its minimum performance state.

The selection criterion for the maximin criterion can be described, in the author's own words, as follows: 


\section{Selection Criterion}

Consider the general decision table in Table 2.2, where there is a choice

between alternative $A_{1}$ and $j$ th alternatives $A_{j}$, where $j=2, \ldots, m$

One should select alternative $A_{1}$ if, and only if:

$$
\min \left(p_{1}\right)>\min \left(p_{j}\right)
$$

Figure 2. 6 Maximin Selection criterion

\subsubsection{Maximax Criterion}

The Maximax criterion can be viewed as the opposite of the Maximin criterion, in the sense that it is completely optimistic (optimistic decision-maker) ${ }^{36}$. The optimistic decision maker is willing to risk high losses for the chance at gaining large rewards. The general philosophy of this criterion assumes that the best outcome possible will happen. With this criterion, the alternative is represented by and selected based on its maximum performance state. The alternatives are ranked based on their potential gain.

The selection criterion for maximax criterion can be described, in the author's own words, as follows:

\section{Selection Criterion}

Consider the general decision table in Table 2.2, where there is a choice between alternative $A_{1}$ and $j$ th alternatives $A_{j}$, where $j=2, \ldots, m$

One should select alternative $A_{1}$ if, and only if:

$\max \left(p_{1}\right)>\max \left(p_{j}\right)$

Figure 2. 7 Maximax Selection criterion

\subsubsection{Hurwicz Criterion}

When the Hurwicz criterion is used, the decision is considered neither completely pessimistic, nor optimistic. Hurwicz ${ }^{37}$ suggests that few would wish to be as pessimistic or optimistic as the Maximin and Maximax criterion suggest. He proposes a optimism- 
pessimism index, $\alpha$, where $0 \leq \alpha \leq 1$, as a measure of the decision-maker's preferences. In this criterion, the coefficient of optimism is multiplied by the maximum performance state and the coefficient of pessimism, 1- $\alpha$, is multiplied by the minimum performance state.

The critical step in the application of this criterion is the determination of $\alpha$. To determine $\alpha$, we employ a method used in utility theory to determine a decision-maker's preference. Specifically, the decision-maker is asked to determine his/her certainty equivalent for a specific lottery, which is a hypothetical situation used to assess the decision-maker's preference ${ }^{10}$. The certainty equivalent is the achievement value at which a designer would be indifferent between receiving the achievement value for certain or receiving the result of the lottery between either getting $v_{0}$ (lowest level of achievement) and $v_{1}$ (highest level of achievement). The decision maker must choose $v_{\text {certain }}$ such that he/she is indifferent to Option A and Option B in Figure 2.9.

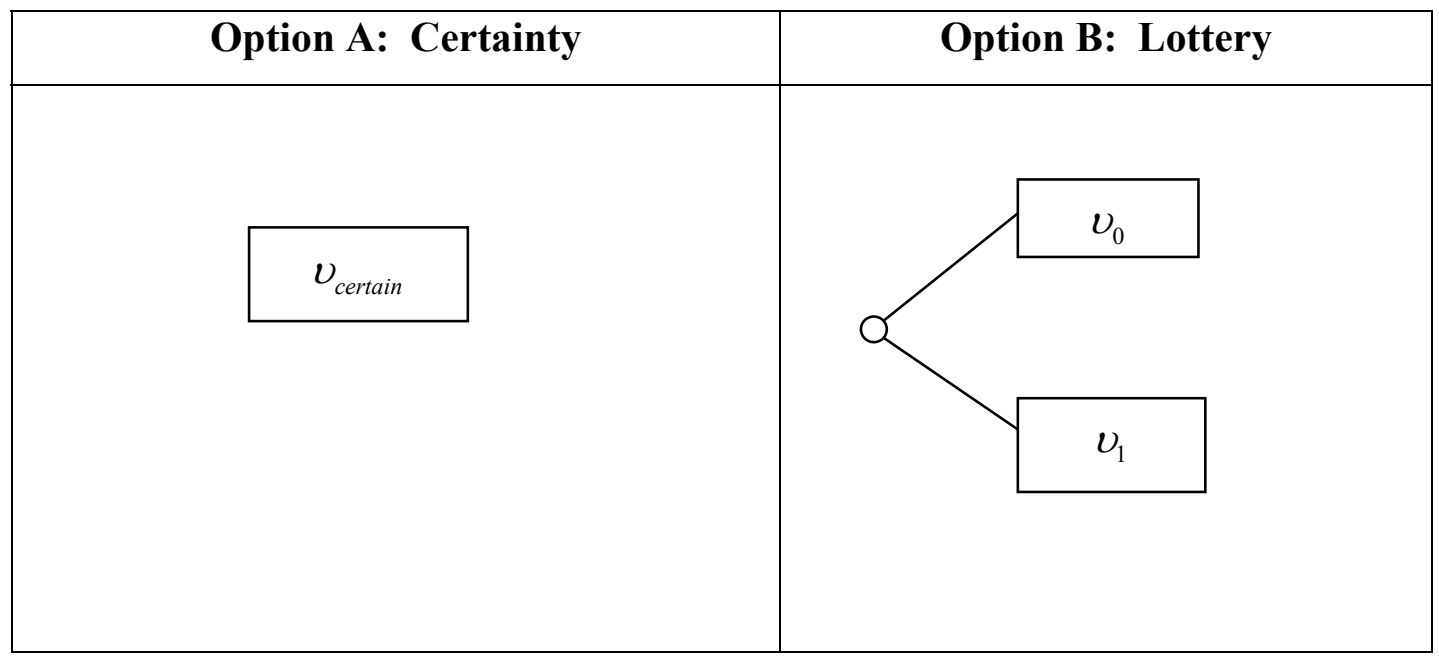

Figure 2. 8 Certainty equivalent determination

Note that $v_{\text {certain }}$ should be normalized according to the scale used for the lottery in Figure 2.8 , where $0<v_{\text {certain }}<1$. Once the value of $v_{\text {certain }}$ is determined, the value $\alpha$ can be determined. This value of $v_{\text {certain }}$ reflects the decision maker's optimism-pessimism index, $\alpha$. 
For clarity on how to determine the decision maker's risk preference, $v_{\text {certain }}$, let us consider the following example. To determine $v_{\text {certain }}$, we consider a lottery between $\$ 0$ and $\$ 100$ and pose the following question: "How much money am I willing to risk in order to gain $\$ 100$, with also having the possibility of getting nothing (\$0)." In most cases in engineering design, decision makers are pessimistic about the future, meaning they are reluctant to risk getting $\$ 0$ for the possibility of getting $\$ 100$. If the decision maker is optimistic about the future, the decision maker is willing to risk getting $\$ 0$ for a chance at winning $\$ 100$. In this example, the decision maker determines he/she is willing to risk $\$ 30$. After normalization, $v_{\text {certain }}$, in this case is equal to 0.3 , therefore $\alpha$ is equal to 0.3 .

For $0.5<\alpha \leq 1$, the decision-maker is considered optimistic, where $\alpha=1$ being completely optimistic (maximax criterion). For $0 \leq \alpha<0.5$, the decision-maker is considered pessimistic, where $\alpha=0$ being completely pessimistic (maximin criterion).

The selection criterion for the Hurwicz criterion can be described, in the author's own words, as follows:

\section{Selection Criterion}

Consider the general decision table in Table 2.2, where there is a choice between alternative $A_{1}$ and $j$ th alternatives $A_{j}$, where $j=2, \ldots, m$

$\alpha=$ optimism-pessimism index, where $0 \leq \alpha \leq 1$

$P_{1}(\alpha)=\alpha \cdot \max \left(p_{1}\right)+(1-\alpha) \cdot \min \left(p_{1}\right)$

and

$P_{j}(\alpha)=\alpha \cdot \max \left(p_{j}\right)+(1-\alpha) \cdot \min \left(p_{j}\right)$

One should select alternative $A_{1}$ if, and only if:

$P_{1}(\alpha)>P_{j}(\alpha)$

Figure 2.9 Hurwicz Selection criterion 


\subsubsection{Laplace Criterion}

The Laplace Criterion, or the equal likelihood criterion, considers all states equally likely (see Section 2.2.2 for further details). The average of the possible outcomes is used as the selection parameter. Equal probability is assigned to each possible outcome.

The selection criterion for Laplace criterion can be described, in the author's own words, as follows:

\section{Selection Criterion}

Consider the general decision table in Table 2.2, where there is a choice between alternative $A_{1}$ and $j$ th alternatives $A_{j}$, where $j=2, \ldots, m$

Let $\mathrm{n}=$ total number of states of nature $(S)$

$P_{1}=\sum_{k=1}^{n} \frac{1}{n} \cdot p_{1, k}$

and

$P_{j}=\sum_{k=1}^{n} \frac{1}{n} \cdot p_{j, k}$

One should select alternative $A_{1}$ if, and only if:

$P_{1}>P_{j}$

Figure 2. 10 Laplace Selection criterion

\subsubsection{Critical Review of Four Selection Criteria}

In reviewing these four criteria, it should be noted that each are quite distinct in principle and can lead to different alternative rankings. For example, consider the $3 \times 3$ decision table in Table 2.3. In this table, we consider the choice between 3 alternatives $(A)$ with performance values at 3 states of nature $(S)$. 
Table 2. 3 Example decision table

\begin{tabular}{c|ccc|cccc|} 
& $S_{1}$ & $S_{2}$ & $S_{3}$ & $\min$ & $\max$ & $\alpha=0.8$ & Laplace \\
\hline$A_{1}$ & 2 & 2 & 0 & 0 & 2 & 1.6 & 1.33 \\
$A_{2}$ & 1 & 1 & 1 & 1 & 1 & 1 & 1 \\
$A_{3}$ & 0 & 4 & 0 & 0 & 4 & 3.2 & 1.33
\end{tabular}

In Table 2.3, the alternative performance representations are also given for the four selection criteria. Based on the Maximin criterion, where the minimum performance state is maximized, Alternative $A_{2}$ should be selected. Based on the Maximax criterion, where the maximum performance state is maximized, Alternative $A_{3}$ should be selected. Based on the Hurwicz criterion and given a decision maker optimism-pessimism index $(\alpha)$ of 0.8 , alternative $A_{3}$ should be selected. With the Laplace criterion, where all states are equally weighted, $A_{1}$ and $A_{3}$ are equally given preference. Given the potentially contradictory results of the different selection criteria, great care must be taken in choosing a selection criterion. The question now becomes, "Which selection criteria should be used to select a RM technology under geometric uncertainty?" In answering this question, let us review each criterion with respect to its limitations in the context of RM.

As seen in the previous example, the Maximin and Maximax criterion, although taking into account the risk preference of the decision maker, are both flawed in the sense that they only consider the decision maker to be either completely pessimistic or optimistic, respectively. It is highly unlikely that a decision maker would want to classify his/herself as either completely optimistic or pessimistic about the future. Additionally, these criteria only consider the maximum (Maximax), or minimum (Maximin), performance states of an alternative, thus neglecting all the other performance information that may be available to the decision maker.

The Hurwicz criterion uses the decision maker's preferences, along with the maximum and minimum performance states of the alternative, as a basis for selection. Although 
this criterion considers both the maximum and minimum performance states as a basis for selection, it is also flawed in the sense that it neglects the other performance information supplied to the decision maker. Also, by basing selection on the decision maker's preference, the selection is considered subjective.

As explained in Section 2.2.3, the Laplace's criterion considers the average performance of the alternatives as a basis for selection. It is flawed in the sense that it assumes that because a decision maker has not assigned a PDF to an event, then he/she must have not done so because there was insufficient reason to indicate that one state was more or less likely to occur than any other state. Thus, this criterion assumes that all events are equally probable (uniform PDF). Although all the performance information is used, it assumes additional information (equal likelihood) that may not scientifically founded.

Back to the question, "Which selection criteria should be used to select a RM technology under geometric uncertainty"? As seen in our discussion above, because of the limitations to each selection criterion, one criterion cannot be deemed 'best' for all cases. Because of this, the author believes that the selection criterion should be chosen on a case-by-case basis. This selection should be based on the type of decision problem considered. This issue is further discussed in Section 5.3.

\subsection{CHAPTER SUMMARY AND VALIDATION}

The role of Chapter 2 was to lay the theoretical foundation to support selection for RM under uncertainty. The three foundational constructs are the selection DSP (section 2.1), uncertainty handling (section 2.2), and selection under epistemic uncertainty (section 2.3) were presented. The literature supporting these constructs was also presented and reviewed.

In Section 2.1, the Selection DSP was introduced along with its word formulation and steps for application. It was concluded that although the selection DSP has many 
advantages, it needs to be extended to consider the types of uncertainty present in Rapid Manufacturing.

In Section 2.2, the two distinct types of uncertainty (aleatory and epistemic) were introduced, as well as several sources of these types of uncertainty that can be found in Rapid Manufacturing. The thesis was scoped to only deal with epistemic uncertainty so as to only address the uncertainty directly inherent to customization. The author also presented literature surrounding the representation of epistemic uncertainty, and it was concluded that epistemic uncertainty should be represented using interval analysis in the case presented in this thesis.

Section 2.3 introduces the notion of selection under epistemic uncertainty where Decision Theory is invoked as a basis for selection under uncertainty. The four main criteria for selection under strict uncertainty (complete ignorance) were presented: Maximin, Maximax, Hurwicz, and Laplace Criterion. It was concluded that the choice of a selection criterion should be done on a case-by-case basis, depending on the decision problem considered.

With respect to verification and validation, this chapter begins our journey of completing the Validation Square. As presented in Section 1.4, Theoretical Structural Validation (TSV) involves checking the individual constructs and assumptions upon which the method is built, as well as checking the internal consistency of the method when combining the individual constructs. In this chapter, the individual constructs of selection DSP, uncertainty handling, and selection under epistemic uncertainty were all introduced and critiqued. In Chapter 3, the latter part of TSV will be considered, where the internal consistency of the method when combining the individual constructs will be evaluated. 


\section{CHAPTER 3 SYNTHESIZING THE CONSTRUCTS OF SELECTION FOR RAPID MANUFACTURING UNDER EPISTEMIC UNCERTAINTY}

\subsection{UNCERTAINTY AND RAPID MANUFACTURING}

Rapid Manufacturing is the use of RP technologies to manufacture end-use products, or finished parts. Given RM's relatively recent introduction, coupled with a growing market for customized products, there exists a need for decision methods that specifically address the needs of RM. As stated in section 2.1, the selection method used in this thesis is selection DSP. Although the selection DSP has many advantages, including its "provision of context, structure, and domain independence" $"$, it does have its limitations which restrict its application with RM. These limitations include its accounting of uncertainty and the decision maker's preferences in the selection process.

In the context of RM, there are several sources of uncertainty that must be considered. In section 2.2, the author introduced aleatory and epistemic uncertainty. Both of these sources exist in all engineering systems, thus they both exist in RM. The sources of aleatory uncertainty in RM systems can be divided into two main categories: machine and material. With respect to the material, some sources of uncertainty include ultimate tensile strength, hardness, elasticity, density, etc. With respect to the machine, some sources of uncertainty include laser scan speed, power density, delay time, etc. These types of uncertainty are unavoidable and inherent to the RM process.

There are also several sources of epistemic uncertainty present in RM. Some potential sources of epistemic uncertainty include: limited understanding of customer preferences, environmental conditions of use, and limited expression of designer preferences. With respect to RM and its ability for customization, limited understanding of customer preferences stands out as one of the main sources of epistemic uncertainty. At the time of investment in a technology, the decision maker (in the decision problem defined for this thesis) lacks information surrounding the customer's preference for a given configuration at the time of production. In other words, the decision maker does not know what 
geometry is going to be produced (unpredictable) in the RM machine until he/she receives the customer's preferences, which is not acquired until after the investment has been made. This type of uncertainty, in the geometric shape of the part, is referred to as geometric uncertainty in this thesis.

Given the assumption of strict uncertainty, the decision maker is assumed to be ignorant to the chances that one outcome will occur over another (what geometry the customer will choose) at the time of investment. In section 2.3.3, cases were made for the use of the Hurwicz or Laplace criterion when performing selection under strict uncertainty, depending on the specific problem being considered. The Hurwicz criterion allows the inclusion of the decision maker's preferences in performing the selection, while the Laplace criterion allows you to select based upon average performance.

In this thesis, selection DSP was extended with two fundamental concepts: uncertainty handling and selection under uncertainty. This extended method is referred to as the Selection for Rapid Manufacturing methodology. Selection for Rapid Manufacturing, presented in section 3.2, takes advantage of the respective advantages of the selection DSP technique, while offsetting its shortcomings with the infusion of uncertainty handling for customization and selection criteria for selection under strict uncertainty.

For scope, geometric uncertainty is the only source of uncertainty that will be considered in this paper. Although the other uncertainties are important, the author will only investigate the effects of the geometric uncertainty on the selection process.

\subsection{A METHOD FOR SELECTION FOR RAPID MANUFACTURING}

\subsubsection{Overview and Description}

The Selection for Rapid Manufacturing technique builds upon the selection DSP, while extending it to account for the uncertainty that is inherent to customization (geometric uncertainty) and providing a method for selection under this uncertainty (Decision Theory under strict uncertainty). 
In the context of selection for RM, the performance measure for each alternative $\left(A_{i}\right)$ is the Merit Function value, or $M F(u)$, where $u$ describes the geometric uncertainty due to customization. The decision table for selection for RM is displayed in Table 3.1.

Table 3. 1 Selection for RM decision table

\begin{tabular}{c|ccc|} 
& $u_{\min }$ & $\cdots$ & $u_{\max }$ \\
\hline$A_{1}$ & $M F_{11}$ & $\cdots$ & $M F_{1 n}$ \\
$\vdots$ & $\vdots$ & $\ddots$ & $\vdots$ \\
$A_{n}$ & $M F_{n 1}$ & $\cdots$ & $M F_{n n}$
\end{tabular}

For selection for RM, $u$ represents the size range of the part being considered, where $u_{\text {min }}$ represents the minimum bound of geometric uncertainty and $u_{\max }$ represents the maximum bound.

\subsubsection{Word Formulation}

In this section, a detailed description of the selection method proposed in this thesis is presented. The solution of the Selection for Rapid Manufacturing selection methodology involves identifying the set of feasible design alternatives, the principle attributes (criteria) influencing selection, and the relative importances of the attributes. In addition, the uncertainty associated with the product being produced with the alternatives and the decision maker's preferences must also be identified. The alternatives are then rated with respect to each attribute, and a merit function value determined. The alternatives are then ranked using the chosen decision criterion. The word formulation for selection for RM is presented in Figure 3.1. 


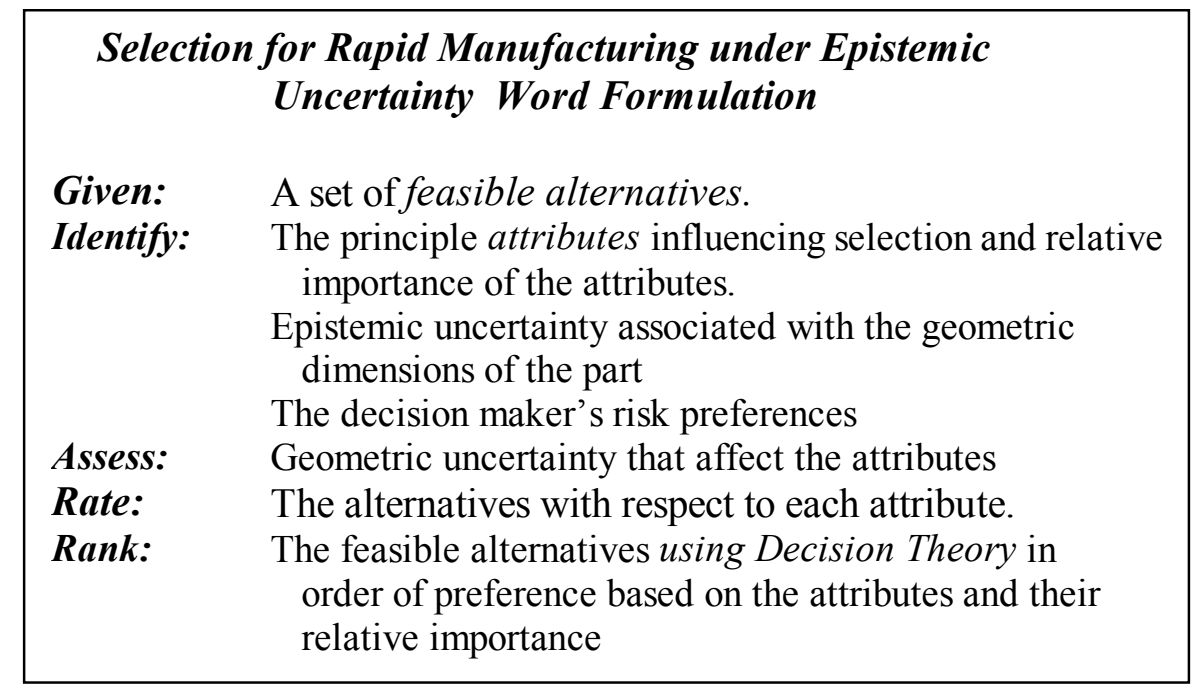

Figure 3. 1 Selection for RM Word Formulation

\subsubsection{Steps for Selection for Rapid Manufacturing under epistemic uncertainty}

A summary of the steps involved in its implementation are presented in Figure 3.2.

\section{Steps of Selection for Rapid Manufacturing (RM) under Epistemic Uncertainty}

1. Characterize the uncertainty involved

a. Qualitatively define the Range of Customization

b. Quantitatively define the uncertainty involved

2. Describe alternatives and provide acronyms

3. Describe each relevant attribute, specify its relative importance, and provide acronyms

4. Specify scales, acceptable range of values, and rate each alternative with respect to each attribute

5. Normalize the attribute ratings

6. Rank and select the alternatives in order of preference using Decision Theory

a. Evaluate the merit functions

b. Evaluate selection values and rank alternatives based on selected Decision Theory criterion

7. Post Solution Analvsis and Verification of Results

Figure 3. 2 Summary of Steps for Selection for Rapid Manufacturing

A detailed description of the 6 steps displayed in Figure 3.2 is presented next.

Step 1. Characterize the uncertainty involved 


\section{Step 1a. Qualitatively define the Range of Customization}

Although this step should be done earlier in the design process, it is good practice to reevaluate the range of customization that is to be offered with a particular product. First, the decision maker should determine the level of customization that is desired. Da

Silveira et al. ${ }^{3}$ has defined eight generic levels of customization, which are displayed in Table 3.2 .

Table 3. 2 Eight Generic Levels of Customization ${ }^{3}$

\begin{tabular}{|l|l|l|}
\hline & Level & $\begin{array}{l}\text { Description } \\
\text { needs }\end{array}$ \\
\hline 8 & Design & $\begin{array}{l}\text { Manufacturing of custom-tailored products within a } \\
\text { finite set of options }\end{array}$ \\
\hline 6 & Fabrication & $\begin{array}{l}\text { Arrangement of custom configurations of modular } \\
\text { components }\end{array}$ \\
\hline 5 & Additional Custom Work & Additional custom work offered at point of delivery \\
\hline 4 & Additional services & $\begin{array}{l}\text { Additional custom services offered at point of } \\
\text { delivery }\end{array}$ \\
\hline 3 & Package and distribution & $\begin{array}{l}\text { Packaging similar products based on general } \\
\text { customer info }\end{array}$ \\
\hline 2 & Usage & $\begin{array}{l}\text { Products that can be adapted by the customer for } \\
\text { different functions }\end{array}$ \\
\hline 1 & Standardization & Standardized products \\
\hline
\end{tabular}

Once the level of customization has been determined, the range of customization can be defined qualitatively. In determining the range of customization, the designer should evaluate and describe which features will be customized for the user. For example, a custom footwear designer (Level 8-Design) may qualitatively define a range of customization as customized insoles and standardized uppers and outsoles. 


\section{Step 1b. Quantitatively define the uncertainty involved}

After the range of customization has been defined qualitatively, a quantitative assessment must be performed. The size ranges for the features can now be defined quantitatively. The designer should define the geometric dimensions and determine which dimensions will be constrained (certain) and which will be customized (uncertain).

As stated before, interval analysis will be used to represent the epistemic uncertainty due to customization. When using the interval representation of epistemic uncertainty, uncertainty is modeled with a closed interval bounded by $z_{\min }$ and $z_{\max }$ (i.e., $Z \in\left[z_{\min }\right.$, $\left.z_{\max }\right]$ ). When epistemic uncertainty is modeled using interval numbers, the design equations are converted to intervals ${ }^{31}$.

\section{Step 2. Describe the alternatives and provide acronyms}

Describe each alternative in words, including its advantages and disadvantages, and provide meaningful acronyms for each. If possible, provide illustrations of the alternatives.

\section{Step 3. Describe each relevant attribute, specify its relative importance and provide acronyms}

The next step in solving the selection DSP is to identify the attributes (criteria) by which the alternatives will be evaluated. Depending on the demands of each problem, the attributes will vary. All relevant attributes should be included. The description of each attribute should be comprehensive and understandable. Also, provide meaningful acronyms for the attributes.

In order to specify the relative importance of the attributes, a pair-wise comparison is used. It is noted that other methods, such as the ranking method, can be used. In the pair-wise comparison method, each of the attributes is rated as better than, worse than, or equal to each of the other attributes. For the comparison, a value of 1 given to the attribute that is better, where a 0 is given to the other attribute. If the attributes are considered equal, both attributes receive a value of zero. Next, the values for each 
attribute are summed and normalized to ensure the relative importance sum to one. To prevent an attribute receiving a total value of zero, a dummy attribute is introduced. In this comparison, the attribute is always preferred to the dummy.

\section{Step 4. Specify scales, acceptable range, and rate the alternatives with respect to each attribute.}

There are four main types of scales: ratio, interval, ordinal, and composite. The type of information available determines the type of scale chosen. The ratio scale is used when quantitative, physically meaningful units are available for an attribute. When an attribute can only be qualified in words, use the ordinal scale. The interval scale is used to convert the words from an ordinal scale to numerical intervals. The composite scale is used when the value of attribute is the result of computations, such as relative importance analysis. Once the scale is determined, the acceptable range of values should also be determined. The decision maker's preference for the higher or lower value of the acceptable range should also be determined for each attribute. If the value is outside of this acceptable range, a rating of 0 (below acceptable range with respect to preference) or 1 (above acceptable range with respect to preference) is given.

In this step, the alternatives are also rated with respect to each attribute. The bounded geometric characteristics (such as part volume, area, etc.) are calculated using interval arithmetic operations on the bounded and constrained geometric dimensions. A sample of these arithmetic operations is presented in Figure 2.5.

The bounded geometric characteristics will be used to determine selection attributes, such as build time, part cost, etc., which will also be intervals once the uncertainty is propagated. The remaining attributes are also rated using scalar values.

\section{Step 5: Normalize the attribute ratings}

Once the alternatives are rated with respect to the attributes, the ratings must be normalized to find a common ground for comparison. When higher values of an attribute rating are preferred, the Eq. 3.1 should be used to normalize the attribute ratings. 


$$
N R_{i j}=\frac{A_{i j}-A_{r, \text { min }}}{A_{r, \text { max }}-A_{r, \text { min }}}
$$

When lower values are preferred, the Eq. 3.2 should be used to normalize the attribute ratings.

$$
N R_{i j}=\frac{A_{r, \text { max }}-A_{i j}}{A_{r, \text { max }}-A_{r, \text { min }}}
$$

where $A_{i j}$ is the attribute rating with respect to alternative j, $A_{r, \max }$ is the maximum value of the acceptable range, $\mathrm{A}_{r \text {,min }}$ is the minimum value of the acceptable range, and $N R_{i j}$ is the normalized rating of the attribute $i$ with respect to alternative $j$.

In the case of uncertainty in the attribute value, $\left[N R_{i j}{ }^{\min }, N R_{i j}{ }^{\max }\right]$ will be defined using Eqs. 3.3 and 3.4.

$$
N R_{i j}{ }^{\min }=\frac{A_{i j}^{\min }-A_{r, \text { min }}}{A_{r, \max }-A_{r, \min }}
$$

and

$$
N R_{i j}^{\max }=\frac{A_{i j}^{\text {max }}-A_{r, \text { min }}}{A_{r, \text { max }}-A_{r, \text { min }}}
$$

when higher values of an attribute rating are preferred. When lower values of an attribute rating are preferred, $\left[N R_{i j}{ }^{\min }, N R_{i j}{ }^{\max }\right]$ will be defined using Eqs. 3.5 and 3.6.

$$
N R_{i j}{ }^{\min }=\frac{A_{r, \text { max }}-A_{i j}{ }^{\text {min }}}{A_{r, \text { max }}-A_{r, \text { min }}}
$$

and

$$
N R_{i j}^{\max }=\frac{A_{r, \text { max }}-A_{i j}^{\text {max }}}{A_{r, \text { max }}-A_{r, \text { min }}}
$$




\section{Step 6: Rank and select the alternatives in order of preference using Decision Theory selection criteria}

Step 6a. Evaluate the merit functions

The merit function values of the alternatives are calculated using Eqs. 3.7 and 3.8.

$$
M F_{j, \min }=\sum_{j=1} I_{i} \cdot N R_{i j}^{\min }
$$

and

$$
M F_{j, \max }=\sum_{j=1} I_{i} \cdot N R_{i j}^{\max }
$$

where $M F_{j}$ is the merit function of alternative $j, I_{i}$ is the relative importance of attribute $i$, and $N R_{i j}$ is the normalized rating of the attribute $i$ with respect to alternative $j$.

\section{Step 6b. Evaluate selection values and rank alternatives based on selected Decision}

\section{Theory criterion}

In this step, the selection criteria from Decision Theory under strict uncertainty will be evaluated with respect to the decision problem. Since the Maximin and Maximax criterion can be derived from the Hurwicz criterion, only the Hurwicz and Laplace criteria will be considered. Based on the choice of the selection criterion, the selection parameters will be determined and the alternatives ranked based on these respective selection criteria. The selection criterion for the Hurwicz criterion is presented in Section 2.3.2.3. The selection criterion for the Laplace criterion is presented in Section 2.3.2.4. The most promising alternative is selected based on the alternative rankings.

In the context of Rapid Manufacturing, for the Hurwicz criterion, $\alpha$ represents the point in the performance interval (Merit Function interval) at which the decision maker will choose to represent the alternative (RM technology) for the purpose of selection. After the geometric uncertainty in the RM decision problem is propagated, the performance of each alternative will also be uncertain. It should be noted that because the performance of the RM technologies corresponds to specific values in the geometric uncertainty range, the decision preference $(\alpha)$ also determines the point in the uncertainty interval that will be used in the selection. In most cases of RM, performance is strongly dependent on part 
size, or volume (i.e., low values of $\alpha$ correspond to high values in the uncertainty interval). This is because larger parts cost more and take more time to build, thus are not efficient in RM machines.

In the case of $\alpha=0$, the decision maker is viewed as pessimistic (about the future), and would want the represent the RM alternative by its minimum bound of performance. This assures that in the case our technologies are running at minimum performance, we are still achieving a certain level of superiority (with respect to the other alternatives). In the case of $\alpha=1$, the decision maker is viewed as optimistic (about the future), whereby he/she would choose to represent each technology by its maximum bound of performance. In most engineering decisions, decision makers are viewed as neither completely pessimistic $(\alpha=0)$ or completely optimistic $(\alpha=1)$. In these cases, the decision maker has a graded preference, whereby $0<\alpha<1$. In order to determine $\alpha$, we believe one should ask the following question: "Given an uncertain performance for my RM technologies, where in the performance interval (which corresponds to the uncertainty interval) should I perform my selection?" In the context of the lottery question in Fig. 2.8, $\alpha$ is considered the point in the performance interval at which the decision makers would be indifferent to receiving that performance level for certain or receiving the lottery between either achieving maximum performance or minimum performance. Knowing that my larger parts will give me the worst performance, and my smaller parts give me best performance, at what point in the performance interval should the decision be made? This tradeoff should be considered when choosing $\alpha$.

\section{Step 7: Post Solution Analysis and Verification of results}

In this step, the results from Step 6 are reviewed and verified. The designer must determine if the results seem logical and reasonable. Verification may involve changing the weighting schemes (relative importances) of the attributes for different scenarios. Once the merit functions are recalculated, the alternative rankings should be compared and evaluated. 


\subsection{CHAPTER SUMMARY AND VALIDATION}

The focus of this thesis is the extension of the selection DSP to account for uncertainty and select under uncertainty. In Chapter 2, the author presented the individual constructs that will be combined to form the selection method described in this thesis. These constructs include selection DSP (Section 2.1), uncertainty handling (Section 2.2), and selection under uncertainty (Section 2.3).

In Chapter 3, these individual constructs were synthesized into the Selection for RM methodology. In Section 3.1, the context for which the selection method was established is presented. This section also presents how the individual constructs will be used in the selection method. In Section 3.2, the Selection for RM methodology is introduced. In this section, the word formulation and the steps for solution are presented.

With respect to verification and validation, this chapter continues our journey towards establishing Theoretical Structural Validity (TSV). As presented in Section 1.4, TSV involves checking the individual constructs and assumptions upon which the method is built, as well as checking the internal consistency of the method when combining the individual constructs. Specifically, this chapter deals with checking the consistency of the overall selection method. In this chapter, we have shown that selection DSP can be extended without any significant change in its formulation. We have also shown that the fundamental axioms of interval analysis and the Decision Theory selection criteria remain intact when used in the context of RM. From this, it can be concluded that the selection method presented in this thesis is structurally sound, therefore, TSV has been established.

In Chapter 4, the author introduces the build time and part cost estimation models used in the selection method proposed for RM. These are the two selection attributes that are most affected by the geometric uncertainty due to customization. The author also introduces the Matlab GUI tool that was developed for build time and part cost estimation. 


\section{CHAPTER 4 BUILD TIME AND PART COST ESTIMATION MODELS}

In this thesis, the author proposes the Selection for Rapid Manufacturing methodology as the framework for selection under geometric uncertainty inherent to RM. In Chapter 3, this methodology was introduced along with its word formulation and steps for implementation. In Step 4 of Selection for Rapid Manufacturing, the alternatives are rated with respect to each attribute. The natural variation due to customization affects two main selection attributes and its alternative ratings: build time and part cost.

Initially, the geometric uncertainty (interval dimensions) is used to calculate the geometric characteristics, such as part volume, part area, etc. These bounded geometric characteristics are then used to determine the selection attributes (build time and part cost) which are highly dependent upon the geometric characteristics. Once the geometric uncertainty is propagated, these selection attributes will also be intervals. Build time and part cost can be seen as the main uncertainty carriers in the selection process, thus careful attention must be paid to their calculations. The build time and cost estimation models developed for Selection for RM are described in Sections 4.2 and 4.3. A Graphical User Interface (GUI) was also developed to implement and test the build time and cost estimation models.

\subsection{THE RAPID PROTOTYPING PROCESS}

The RP process consists of 5 main steps ${ }^{5}$ :

Step 1. Create the CAD model - The first step in the RP process involves modeling the object using a CAD software package. Some common software packages include Solidworks, Pro/Engineer, I-DEAS, etc.

Step 2. Convert the CAD model to .STL file format - Once the CAD model is built using a software package, the next step is to convert the CAD model into .STL file format. .STL format represents the solid model using an assembly of planar triangles. The file contains the coordinates of vertices and the direction of the outward normal for each 
triangle ${ }^{5}$. The .STL file is used to establish consistency in the representation of models for use in RP.

Step 3. Slice the .STL file - The third step is to slice the .STL model into thin crosssections. This slicing thickness depends on the build style. At this step, support structures are also generated to support overhangs and thin-walled sections during the build.

Step 4. Construct the part model - The fourth step of the RP process is the construction of the model. This part of the process is technology specific, but all technologies follow a basic pattern. In this basic build process, the machine warms up and each cross-section is built (layer-by-layer) until the part is finished.

Step 5. Clean and finish the model - After the model is built, the final step in the RP process is to clean and finish the part. These processes are also technology-specific, since each technology requires different materials and processes to finish these materials.

In the RP process, steps 1-3 are considered pre-processing steps, and step 5 is considered post-processing. A flowchart of the RP process is displayed in Figure 4.1. The build time discussed in this chapter is based only on Step 4 of the RP process (part build), while the part cost model is based on the entire RP process. 


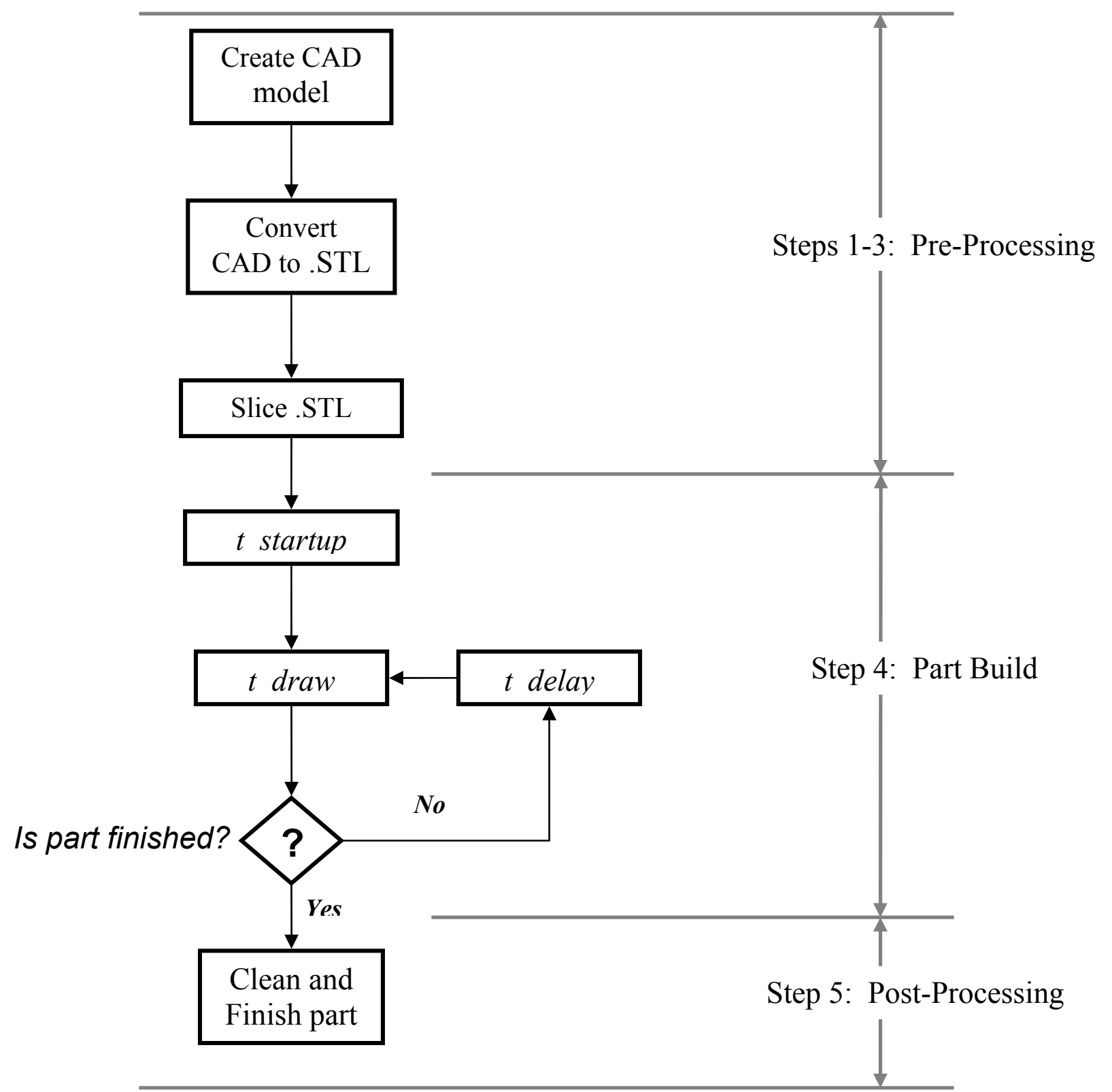

Figure 4. 1 RP build process

\subsection{BUILD TIME ESTIMATION (BTE)}

Build time in this thesis is defined as the total time required to build the part(s). As stated earlier, build time estimation only considers step 4 of the RP build process in Figure 4.1. The build time estimation (BTE) method presented in this thesis is considered parametric, meaning that its solution depends only on the values input for the parameters. Given that, this BTE can be used universally across the different RM technologies. It should also be 
noted that because of this parameterization, the accuracy of the solution significantly depends on the accuracy of the input parameters.

For RM, it is assumed that a full vat is used for manufacture. Therefore, the time to build the full vat is averaged among the number of parts per vat, giving an average build time per part. The BTE model developed for Selection for RM is described in the following sections.

\subsubsection{Build Time Correction Factor}

There are two main part characteristics that need to be determined prior to build time and cost estimation for RM technologies: the calculated part volume (vol_act) and the volume of the bounding box of the part $\left(V_{b b}\right)$. The dimensions corresponding to these part characteristics are as follows: actual part volume, part length (maximum), part width $(\max )$, and part height $(\max )$. These parameters are used to determined a factor $(\rho)$, which is the ratio of part volume to the part bounding box ${ }^{38}$. Pham et al. ${ }^{38}$ use this factor to determine the distribution of the sintering (scan) area, which is very important to characterize. They note the high dependency of the scan time on not only the sintering area, but also the distribution of the area. This is due to the change in velocity from the laser scanning and the laser jump. To account for the distribution of area and the velocity change, Pham et al. modified the average scan area by a factor $(f(\rho))$, where

$$
f(\rho)=\rho^{*} \mathrm{e}^{\alpha(1-\rho)} \quad f(\rho) \leq 1,0 \leq \rho \leq 1
$$

where $\alpha$ is empirically determined from experimental trials. This allows for adjustment of the factor using experimental results. The empirically determined factor, $\alpha$, will change from machine to machine within a given technology.

It is also believed that this factor will make the build time estimation method developed as part of this work more accurate given the main assumption, which is as follows: Estimation of the build time using this method assumes that the scan draw mechanism will touch every point of the cross-section of the part. 


\subsubsection{Build Time Estimation Model}

The build time is the total time required to build the part. The author believes that the build time can be divided into 3 main contributors: draw time $\left(t_{\text {draw }}\right)$, delay time $\left(t_{\text {delay }}\right)$, and startup time $\left(t_{\text {startup }}\right)$. The build time is determined using Equation 4.2.

$$
t_{\text {build }}=t_{\text {draw }}+t_{\text {delay }}+t_{\text {startup }}
$$

There were several methods examined to calculate the build time, each with a different interpretation of the calculation of the scan time. The term 'draw' is used in the model to describe the material deposition, solidifying, and sintering processes for the FDM, SLA, and SLS processes, respectively. For the model discussed in this paper, the draw time is calculated as a function of the scan distance and the scan velocity, making it dependent upon the part geometry and the machine parameters. It is assumed that the laser must scan every point on the layer, therefore the scan distance can be determined from the area of the layer and the diameter of the draw mechanism. The draw time is determined from summing the time to build the part layers and the time to build the support layers, as seen in Equation 4.3.

$$
\begin{aligned}
t_{\text {draw }}=t_{\text {one_part_layer }} & \left(N_{\text {part_layers }}\right)+t_{\text {one_support_layer }}\left(N_{\text {support_layers }}\right) \\
& =\frac{N_{\text {scan }} \cdot A_{\text {avg }}}{D_{\text {scan }} \cdot V_{\text {avg }} \cdot \text { Hatch }}\left(\frac{z_{\text {height }}}{t_{\text {layer }}}\right)+\frac{N_{\text {scan_supp }} \cdot A_{\text {avg }} \cdot \text { supp_factor }}{D_{\text {scan }} \cdot V_{\text {avg }} \cdot \text { Hatch }}\left(\frac{z_{\text {supp }}}{t_{\text {layer }}}\right)
\end{aligned}
$$

where $N_{\text {scan }}$ is the number of scans, $D_{\text {scan }}$ is the diameter of the scan (draw) mechanism, $V_{a v g}$ is the average velocity of the scan (draw) mechanism, supp_factor is the volume ratio of the supports to a fully-scanned area, and Hatch is the spacing between parallel scans.

The total build height $\left(z_{\text {height }}\right)$ and the support height $\left(z_{\text {supp }}\right)$ in the equation takes into account parts built at an angle $(\theta)$ to prevent crashes in the build. The total build height 
and the support height are determined using Equations 4.4 and 4.5. A schematic of the total build height calculation is displayed in Figure 4.2.

$$
z_{\text {total }}=l_{\text {part }} \cdot \sin \theta+z_{\text {part }} \cdot \cos \theta
$$

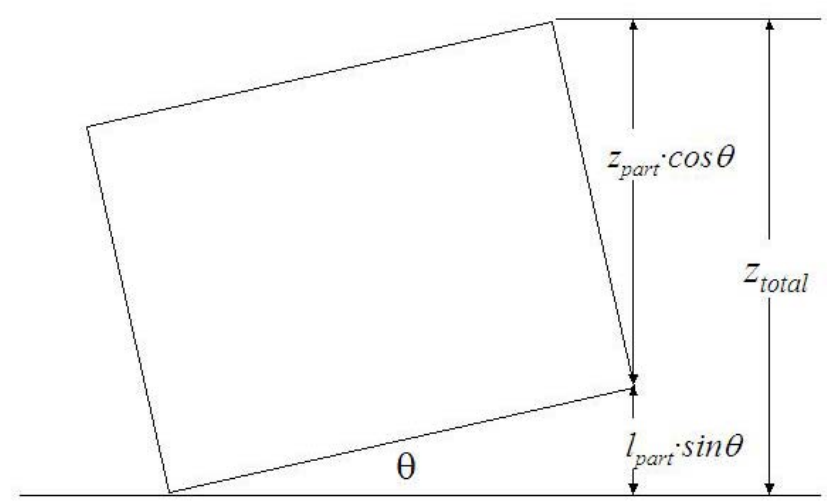

Figure 4. 2 Total Build Height

$$
z_{\text {supp }}=z_{\text {supp_min }}+\frac{l_{\text {part }} \sin \theta}{2}
$$

The average area $\left(A_{\text {avg }}\right)$ can be corrected using the area factor as follows [Pham et al., 2000]:

$$
A_{\text {avg }}=\frac{V_{b b} \cdot f(\rho)}{z_{\text {part }}}=A_{a c t} \cdot \frac{f(\rho)}{\rho}
$$

where $V_{b b}$ is the volume of the bounding box of the part and $A_{a c t}$ is the uncorrected average area of the part. As the amount of empty space in a given volume is increased, $\rho$ (and the factor, $f(\rho))$ decrease at different rates, with the factor $f(\rho)$ decreasing at a slower rate. This means that the more empty space present in a volume, the more the average area is positively corrected. In essence, the factor increases the average area to account for the laser jumps caused by an empty space in a cross-section. I believe this is a reasonable assumption. 
The average scan velocity is also determined using the factor as follows:

$$
V_{a v g}=V_{\text {scan }} f(\rho)+V_{\text {jump }}[1-f(\rho)]
$$

The delay time is defined as the total time between scans. The delay time is determined using Equation 4.8.

$$
t_{\text {delay }}=\left(\frac{z_{\text {total }}}{t_{\text {layer }}}\right)\left(t_{\text {draw_delay }}+t_{\text {stg_down }}+t_{\text {stg_delay }}+t_{\text {stg_up }}+t_{\text {sweep }}+t_{\text {swp_delay }}\right)
$$

where:

$t_{\text {draw_delay }}$ - delay after draw, but before the stage moves

$t_{\text {stg_down }}$ - time for stage to move down

$t_{\text {stg_delay }}$ - delay time between stage movements

$t_{\text {stg_up }}$ - time for stage to move up

$t_{\text {sweep }}$ - time for material sweep

$t_{\text {swp_delay }}$ - delay between material sweep and draw

\subsubsection{Build Time Estimation for Rapid Manufacturing}

The aforementioned build time is the build time for building one part. If multiple parts are considered (as in Rapid Manufacturing), then the maximum number of parts in a single build $\left(N_{\text {build }}\right)$ must be computed as follows:

$$
N_{\text {build }}=\frac{L_{\text {vat }}}{\left(L_{\text {part }}+\text { gap }\right)} \cdot \frac{W_{\text {vat }}}{\left(W_{\text {part }}+\text { gap }\right)} \cdot \frac{H_{\text {vat }}}{\left(H_{\text {part }}+\text { gap }\right)}
$$

The scan time would then be computed as follows:

$$
t_{\text {scan }}=\frac{N_{\text {build }} \cdot N_{\text {scan }} \cdot A_{\text {avg }}}{D_{\text {scan }} \cdot V_{\text {avg }} \cdot H a t c h}\left(\frac{z_{\text {height }}}{t_{\text {layer }}}\right)+\frac{N_{\text {build }} \cdot N_{\text {scan_supp }} \cdot A_{\text {avg }} \cdot \text { supp _factor }_{\text {far }}}{D_{\text {scan }} \cdot V_{\text {avg }} \cdot H a t c h}\left(\frac{z_{\text {supp }}}{t_{\text {layer }}}\right)
$$


In addition to the need for build time estimation for selection, accurate part cost estimation is also needed.

\subsection{PART COST ESTIMATION}

Part cost is defined as the average total cost to build each part. This part cost (PC) model in this thesis is considered parametric, where the solution is a function of the input parameters. This also means that because of this parameterization, the accuracy of the solution significantly depends on the accuracy of the input parameters. For RM, a full vat for manufacture is assumed. Therefore, the cost to build the full vat is averaged among the number of parts per vat, giving an average part cost. The PC developed for Selection for RM is described in the following sections.

To satisfy the need for a parametric cost model for RP/RM technologies, the cost model (displayed in the Figure 4.3) was developed. The model is quantitative, meaning that it requires hard information from the machine and part.

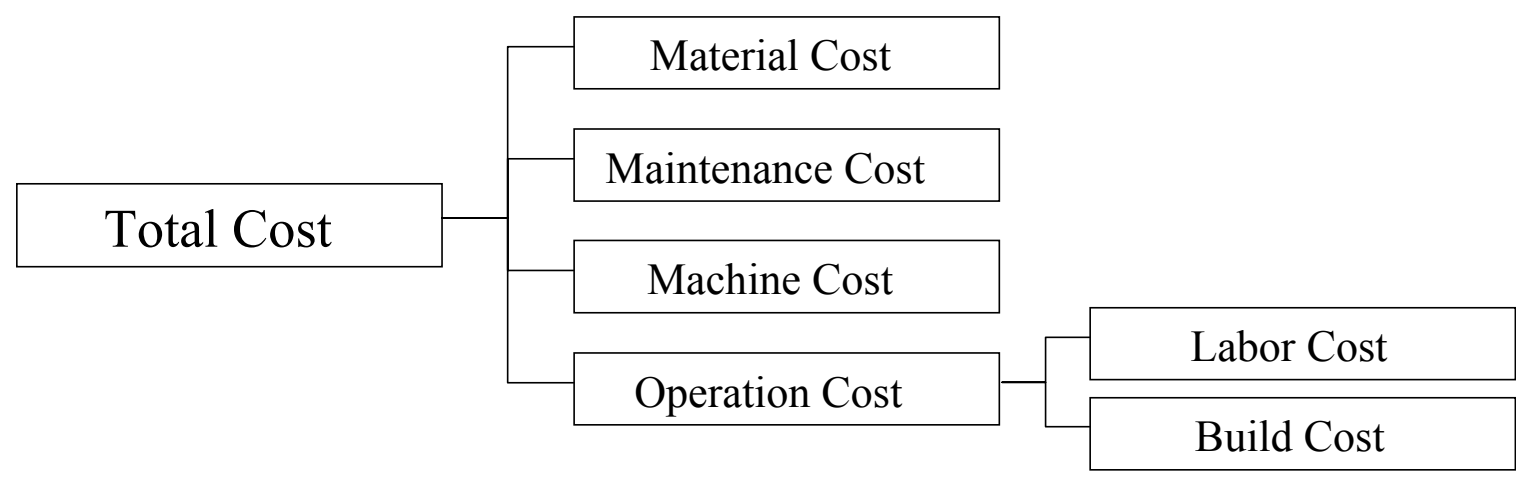

Figure 4. 3 Parametric Cost Model

As displayed in the Figure 4.3, the total cost per part is divided into 4 parts: material, maintenance, machine, and operation cost. Material cost is defined as the total direct cost for materials in the RP process. The maintenance cost is the average annual maintenance cost for the each machine, usually predetermined in the form of a maintenance contract with the manufacturer. The machine cost is the annual cost of the RP machine, without 
depreciation taken into account. Depreciation is the decreasing value of a product over its life. For this model, it is assumed that the part is produced in year 1, where depreciation is not a factor. The operation cost is the cost for operating the RP machine. Operation cost includes the cost of labor and the cost for the machine to build a specified part based on a given labor and machine rate. For the purposes of the model, all costs are on a per part basis. The MATLAB code for the cost model is presented in the Appendix.

\subsubsection{Material Cost}

The material cost represents the total direct cost for materials in the model. In RP/RM, this direct cost is the cost of the build material (resin, etc.). In the model, the material cost per part is determined using Equation 4.11.

$$
C_{\text {material }}=(\text { vol_part } \cdot \text { dens }(1+\text { supp_factor })) \text { MatC }
$$

$M a t C$ represents the cost of material per unit weight. Part volume (vol_part) and the material density (dens) are used to calculate the weight of the part(s) being built. The support factor (supp factor) was included to approximate the volume of material used to build the supports. The support factor varies depending on the technology used. $C_{\text {material }}$ has the units of (\$/part).

\subsubsection{Maintenance Cost}

The maintenance cost represents the annual cost for maintenance of the RP technologies. This maintenance cost (MaintC) is usually on a per year contract term with the manufacturer. This cost per part is determined in the model using Equation 4.12.

$$
C_{\text {maintenance }}=\frac{\text { Maint } C \cdot N_{\text {mach }}}{365 \cdot N_{p p d}}
$$

where $N_{\text {mach }}$ is the number of machines used. The number of parts per day, $N_{p p d}$, can be determined using the number of parts per build ( $\left.N_{\text {build }}\right)$ and the build time of the part(s). 
$C_{\text {maintenance }}$ has the units of (\$/part). The maintenance cost can also be approximated as $10 \%$ of the machine cost ${ }^{6}$.

\subsubsection{Machine Cost}

The machine cost represents the annual cost of owning the RP technology. The machine cost per part is determined using Equation 4.13.

$$
C_{\text {machine }}=\frac{M a c h C \cdot N_{\text {mach }}}{365 \cdot u l \cdot N_{p p d}}
$$

In the model, the total machine cost (MachC) is divided by the useful life of the machine $(u l) . C_{\text {machine }}$ has the units of (\$/part).

\subsubsection{Operation Cost}

The operation cost represents the total cost for operating the machine. The operation cost is determined using Equations 4.14-16.

$$
\begin{gathered}
C_{\text {operation }}=C_{\text {build }}+C_{\text {labor }} \\
C_{\text {labor }}=\left(t_{\text {preproc }}+t_{\text {prostproc }}\right) \text { tech_rate } \\
C_{\text {build }}=t_{\text {build }} \text { Mach_rate }
\end{gathered}
$$

The labor cost and the build cost are both determined using predetermined rates (technician rate and machine rate) that are given appropriate values by the user for the $\mathrm{RP} / \mathrm{RM}$ technology selected. The machine rate (Mach_rate) is the hourly rate associated with running the technology (electricity, floor space, etc.). The labor cost is determined by the time before and after the build and the build cost is determined by the time during the build. It is assumed that a technician is not needed for the build. $C_{\text {build }}$ has the units of (\$/part). 


\subsection{SPECIFIC RM BUILD TIME AND PART COST MODELS}

Using the general build time and part cost models presented in Sections 4.2 and 4.3, technology-specific models can be developed. Since the models are parametric, they can be easily adapted for each technology by varying the models' parameters. The catalog of parameters used in the build time and part cost models are as listed, along with descriptions.

Machine parameters:

L_vat length of RP vat (x-direction) (mm)

H_vat height of RP vat (z-direction) (mm)

$W_{-}$vat $\quad$ width of RP vat (y-direction) $(\mathrm{mm})$

ul useful life of machine (yrs)

Material Characteristics (coupled with machine in this project)

dens material density $\left(\mathrm{g} / \mathrm{cm}^{3}\right)$

MatC material cost per mass $(\$ / \mathrm{kg})$

Material Deposition parameters:

$D_{\text {scan }} \quad$ scan (draw) diameter (length)

hatch percentage overlap of scans (\%)

$V_{\text {jump }} \quad$ jump velocity $(\mathrm{mm} / \mathrm{s})$

$V_{\text {scan }} \quad$ scan $($ draw) velocity $(\mathrm{mm} / \mathrm{s})$

$t_{\text {layer }} \quad$ layer thickness $(\mathrm{mm})$

Scan profile parameters:

$N_{\text {scan }} \quad$ number of times the given surface is scanned (drawn) for parts

$N_{\text {scansupp }} \quad$ number of time the given surface is scanned (drawn) for supports

supp_factor factor used to account for inclusion of supports (\%)

$z_{\text {supp }} \quad$ minimum height of supports ( $\mathrm{mm}$ )

Machine Time parameters: 


$\begin{array}{ll}t_{\text {preproc }} & \text { time for preprocessing operations by the technician (hrs) } \\ t_{\text {prostproc }} & \text { time for postprocessing operations by the technician (hrs) } \\ t_{\text {draw_delay }} & \text { delay after draw, but before the stage moves } \\ t_{\text {stg_down }} & \text { time for stage to move down } \\ t_{\text {stg_delay }} & \text { delay time between stage movements } \\ t_{\text {stg_up }} & \text { time for stage to move up } \\ t_{\text {sweep }} & \text { time for material sweep } \\ t_{\text {swp_delay }} & \text { delay between material sweep and draw } \\ t_{\text {draw }} & \text { total time for scanning (drawing) part (s) } \\ t_{\text {startup }} & \text { machine warmup/setup time (s) } \\ t_{\text {sweep }} & \text { time for material deposition sweep (s) } \\ t_{\text {delay }} & \text { total time between scans (s) }\end{array}$

\section{Cost parameters:}

Mach_rate cost of operating the machine per hour $(\$ / \mathrm{hr})$

MachC cost of machine (\$)

MaintC yearly maintenance cost per machine (\$)

tech_rate technician rate per hour $(\$ / \mathrm{hr})$

In this section, we consider the three main RP process available in the current market: Stereolithography, Selective Laser Sintering, and Fused Deposition Modeling.

\subsubsection{Stereolithography (SLA)}

SLA, manufactured by 3D Systems, is the most widely used RP process. SLA uses a photosensitive polymer that hardens when exposed to ultraviolet light. A schematic of the SLA process is displayed in Figure 4.4. 


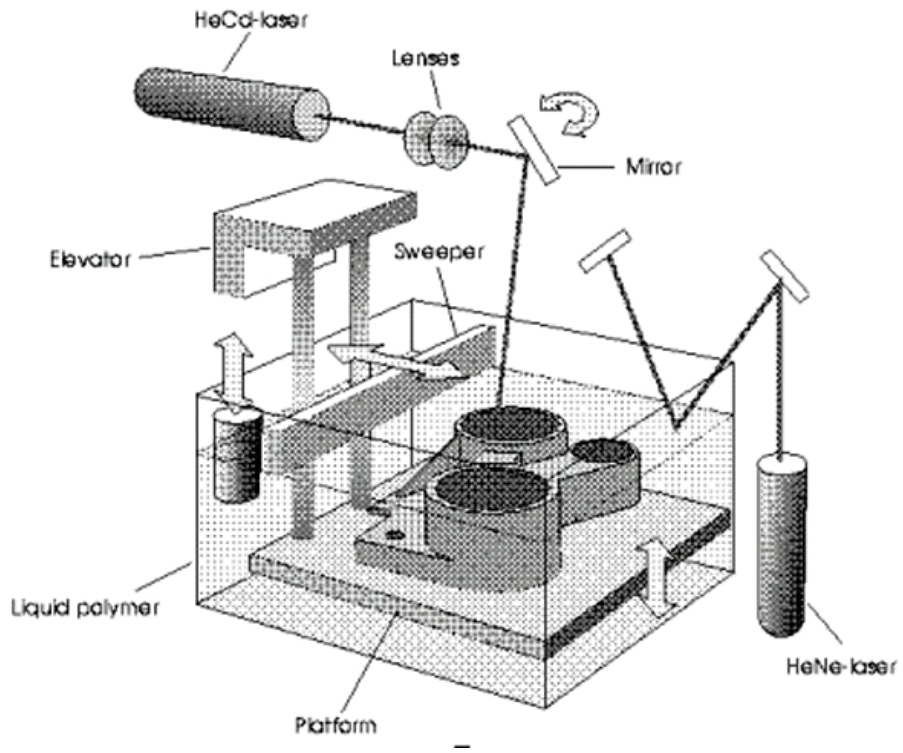

Figure 4. 4 Schematic of Stereolithography process ${ }^{5}$

As displayed in Figure 5.6, a highly focused UV laser is used to trace out the model's cross-section. With the use of an elevator, the platform (upon which the part is built) is moved down one-layer thickness to expose another layer of photopolymeric resin. The sweeper is then used to level the resin layer and evenly coat the previous layer of solidified resin. The laser then traces out the next cross-section of the model, and the process continues until a solid part is manufactured. The build time model for the SLA process is displayed in Figure 4.5.

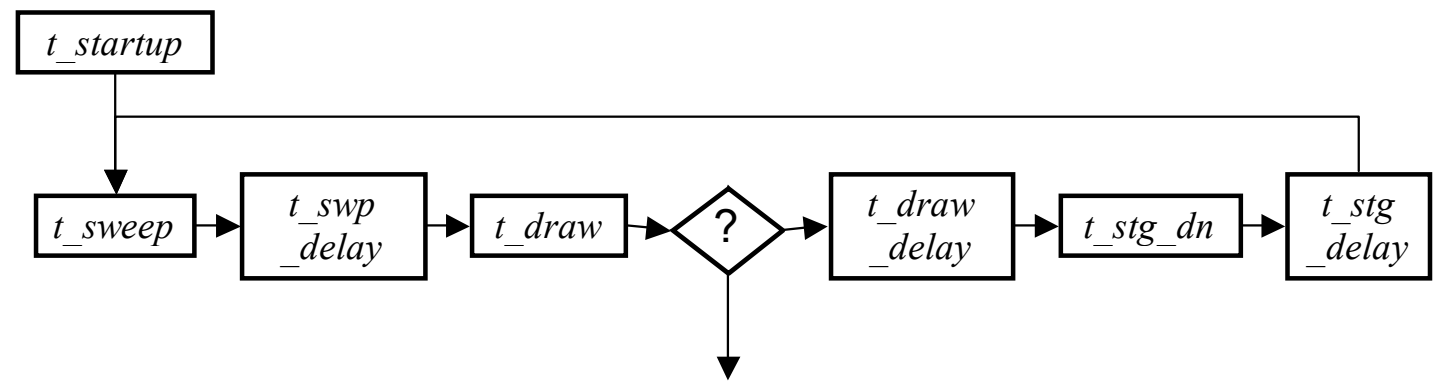

Figure 4. 5. SLA Build Time Model

Given the build time model in Figure 4.5, the parameters used for SLA build time and cost estimation are displayed in Table 4.1. The SLA machines considered in this thesis 
are as the SLA 250, SLA 3500, SLA 7000, and the SLA viper machine, all by 3D systems.

Table 4. 1 SLA Model Parameters

\begin{tabular}{|c|c|c|c|c|}
\hline & SLA 250 & SLA 5000 & SLA 7000 & SLA viper \\
\hline L_vat (mm) & $250^{6,39}$ & $508^{39}$ & $508^{39}$ & $250^{39}$ \\
\hline$W$ vat $(\mathrm{mm})$ & 250 & 508 & 508 & 250 \\
\hline H_vat (mm) & 250 & 584 & 584 & 250 \\
\hline $\operatorname{MachC}(\$ * 1000)$ & $169^{6}$ & $500^{6}$ & $800^{6}$ & $179^{6}$ \\
\hline $\operatorname{Maint} C(\$ * 1000 / y r)$ & \multicolumn{4}{|c|}{$0.1 * \operatorname{Mach}^{6}$} \\
\hline Mach_rate $(\$ / h r)$ & \multicolumn{4}{|c|}{$25[\mathrm{~A}]$} \\
\hline tech_rate $(\$ / h r)$ & \multicolumn{4}{|c|}{$10[\mathrm{~A}]$} \\
\hline $\bar{U} l(y r s)$ & \multicolumn{4}{|c|}{$7[\mathrm{~A}]$} \\
\hline$N_{\text {mach }}$ & \multicolumn{4}{|c|}{$1[\mathrm{~A}]$} \\
\hline$D_{\text {scan }}(\mathrm{mm})$ & $0.25^{39}$ & $0.25^{39}$ & 0.5083 avg. ${ }^{39}$ & $0.25^{39}$ \\
\hline$V_{\text {scan }}(\mathrm{mm} / \mathrm{s})$ & 762 (typical) ${ }^{39}$ & $2500\left(0.5^{*} \max \right)^{39}$ & 3015 (avg.) & 5000 (typical) $^{39}$ \\
\hline$V_{\text {jump }}(\mathrm{mm} / \mathrm{s})$ & \multicolumn{4}{|c|}{$2^{*} V_{\text {scan }}[\mathrm{A}]$} \\
\hline hatch & \multicolumn{4}{|c|}{$0.5[\mathrm{~T}]$} \\
\hline$t_{\text {laver }}(\mathrm{mm})$ & \multirow{2}{*}{\multicolumn{4}{|c|}{$\frac{0.1^{39}}{2[\mathrm{~T}]}$}} \\
\hline$N_{\text {scan }}$ & & & & \\
\hline$N_{\text {scansupp }}$ & \multicolumn{4}{|c|}{$1[\mathrm{~T}]$} \\
\hline$z_{\text {supp }}(\mathrm{mm})$ & \multicolumn{4}{|c|}{$0.1016[\mathrm{~T}]$} \\
\hline supp factor & \multirow{2}{*}{\multicolumn{4}{|c|}{$\frac{0.3[\mathrm{~A}]}{2[\mathrm{~A}]}$}} \\
\hline$t_{\text {draw delav }}(s)$ & & & & \\
\hline$t_{s t g \text { down }}(s)$ & \multicolumn{4}{|c|}{$10[\mathrm{~A}]$} \\
\hline$t_{\text {stg delav }}(s)$ & \multicolumn{4}{|c|}{$2[\mathrm{~A}]$} \\
\hline$t_{\text {sweep }}(s)$ & \multicolumn{4}{|c|}{$10[\mathrm{~A}]$} \\
\hline$t_{s t g \text { иp }}(s)$ & \multicolumn{4}{|c|}{0} \\
\hline$t_{\text {swp delay }}(s)$ & \multirow{2}{*}{\multicolumn{4}{|c|}{$\begin{array}{l}2[\mathrm{~A}] \\
1[\mathrm{~A}]\end{array}$}} \\
\hline$t_{\text {preproc }}(h r)$ & & & & \\
\hline$t_{\text {prostproc }}(h r)$ & \multicolumn{4}{|c|}{$\frac{\frac{1[\mathrm{~A}]}{2[\mathrm{~A}]}}{\frac{1}{2}}$} \\
\hline$t_{\text {startup }}(\mathrm{hr})$ & \multicolumn{4}{|c|}{$0.5[\mathrm{~A}]$} \\
\hline MatC (\$/kg) & \multicolumn{4}{|c|}{$200^{6}$} \\
\hline $\operatorname{Dens}\left(\mathrm{g} / \mathrm{cm}^{3}\right)$ & \multicolumn{4}{|c|}{$1.1[\mathrm{~A}]$} \\
\hline
\end{tabular}

In Table 4.1, the term ' $\mathrm{T}$ ' denotes a value that is typical of what you would find in the SLA process, though not directly quoted. The term 'A' denotes values that were approximated based on available information. Using the parameters in Table 4.1, build time and part cost were calculated for the SLA technologies. 


\subsubsection{Selective Laser Sintering (SLS)}

SLS, also manufactured by 3D Systems, uses a powdered material, such as nylon, elastomer, metal, etc., as its working material. The laser is used to fuse the powdered material into a solid layer. As displayed in Figure 4.6, a laser is used to trace the model cross-section. Once the elevator moves the object down one layer thickness, the leveling roller is used to recoat the part vat with powder. This process is repeated until a solid part is manufactured.

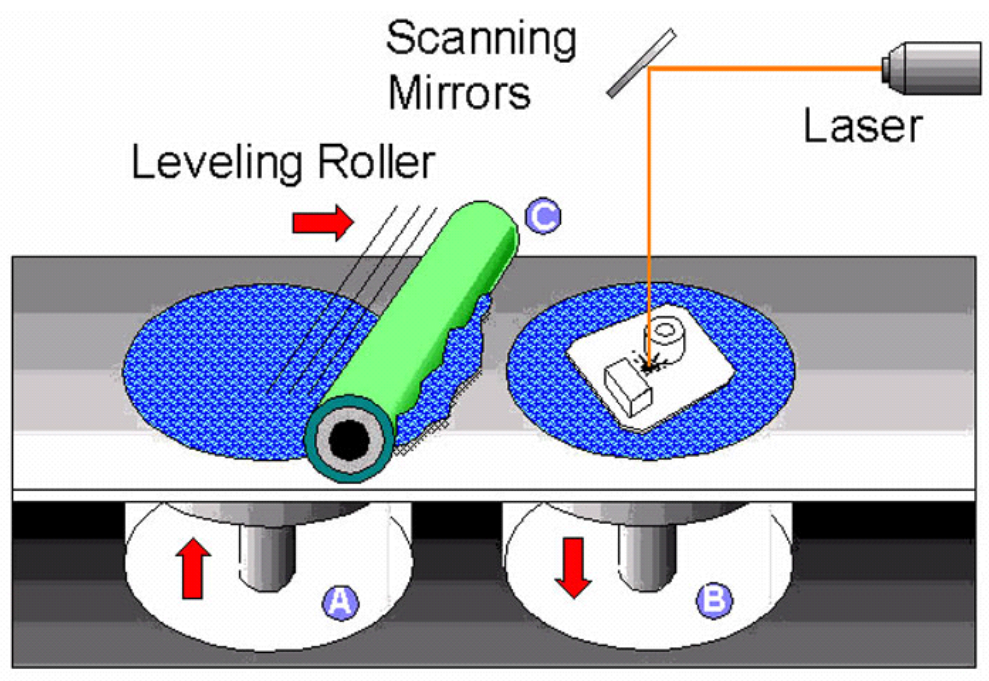

Figure 4. 6 Schematic of SLS system ${ }^{5}$

The build time model for the SLS process is displayed in Figure 4.7.

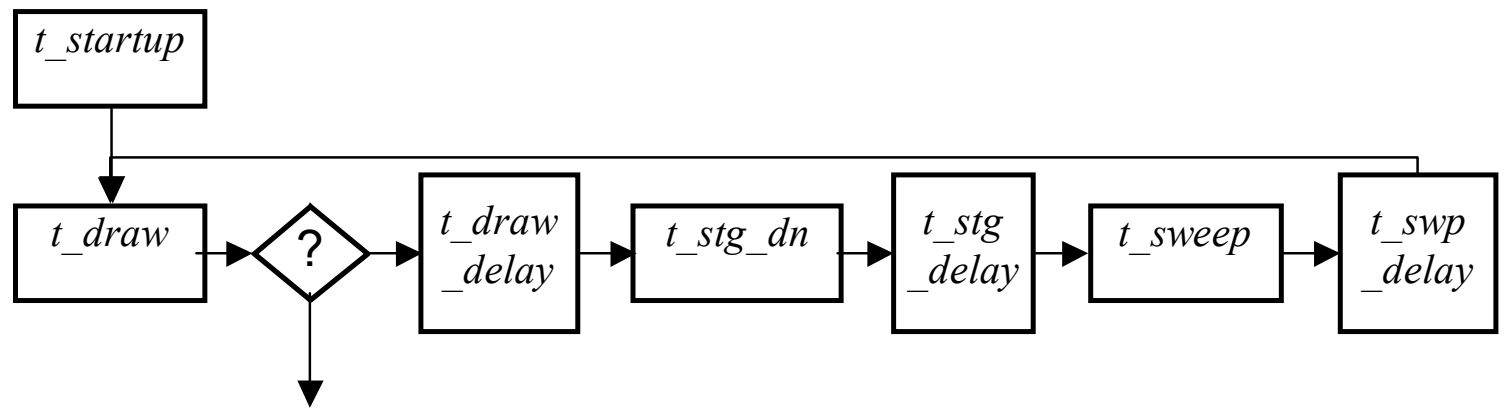

Figure 4. 7 SLA Build Time Model

Given the build time model in Figure 4.7, the parameters used for SLS build time and cost estimation are displayed in Table 4.2. The SLS machines considered in this thesis are the SinterStation HiQ and HiQ+HS series, both by 3D systems. 
Table 4. 2 SLS Model Parameters

\begin{tabular}{|c|c|c|}
\hline & HiQ & $\mathrm{HiQ}+\mathrm{HS}$ \\
\hline L_vat $(\mathrm{mm})$ & $381^{39}$ & $381^{39}$ \\
\hline W_vat $(\mathrm{mm})$ & 330 & 330 \\
\hline$H \_$vat $(\mathrm{mm})$ & 457 & 457 \\
\hline $\operatorname{MachC}(\$)$ & $270^{40}$ & $325^{40}$ \\
\hline MaintC (\$) & \multicolumn{2}{|c|}{$0.1 * \operatorname{MachC}^{6}$} \\
\hline Mach_rate $(\$ / h r)$ & \multicolumn{2}{|c|}{$25[\mathrm{~A}]$} \\
\hline tech_rate $(\$ / h r)$ & \multicolumn{2}{|c|}{$10[\mathrm{~A}]$} \\
\hline$U l(y r s)$ & \multicolumn{2}{|c|}{$7[\mathrm{~A}]$} \\
\hline$N_{\text {mach }}$ & \multicolumn{2}{|c|}{$1[\mathrm{~A}]$} \\
\hline$D_{\text {scan }}(\mathrm{mm})$ & \multicolumn{2}{|c|}{$0.6^{38}$} \\
\hline$V_{\text {scan }}(\mathrm{mm} / \mathrm{s})$ & $2500(0.5 * \max )^{39}$ & $5000\left(0.5 *^{\max }\right)^{38}$ \\
\hline$V_{\text {jump }}(\mathrm{mm} / \mathrm{s})$ & $5000 \max ^{38}$ & $10000 \max ^{38}$ \\
\hline hatch & \multicolumn{2}{|c|}{$1[\mathrm{~T}]$} \\
\hline$t_{\text {layer }}(\mathrm{mm})$ & \multicolumn{2}{|c|}{$0.1[\mathrm{~T}]$} \\
\hline$N_{\text {scan }}$ & \multicolumn{2}{|c|}{$1[\mathrm{~T}]$} \\
\hline$N_{\text {scansupp }}$ & \multicolumn{2}{|c|}{$1[\mathrm{~T}]$} \\
\hline$z_{\text {supp }}(\mathrm{mm})$ & \multicolumn{2}{|c|}{$0.4\left(4 * t_{\text {layer }}\right)[\mathrm{T}]$} \\
\hline supp factor & \multicolumn{2}{|c|}{0} \\
\hline$t_{\text {draw delay }}(s)$ & \multicolumn{2}{|c|}{$2[\mathrm{~A}]$} \\
\hline$t_{\text {stg down }}(s)$ & \multicolumn{2}{|c|}{$5[\mathrm{~A}]$} \\
\hline$t_{\text {stg delay }}(s)$ & \multicolumn{2}{|c|}{$2[\mathrm{~A}]$} \\
\hline$t_{\text {sweep }}(s)$ & \multicolumn{2}{|c|}{$9.5[\mathrm{~A}]$} \\
\hline$t_{\text {stg up }}(s)$ & \multicolumn{2}{|c|}{0} \\
\hline$t_{\text {swp delay }}(s)$ & \multicolumn{2}{|c|}{$2[\mathrm{~A}]$} \\
\hline$t_{\text {preproc }}(h r)$ & \multicolumn{2}{|c|}{$1[\mathrm{~T}]$} \\
\hline$t_{\text {prostproc }}(h r)$ & \multicolumn{2}{|c|}{$1[\mathrm{~T}]$} \\
\hline$t_{\text {startup }}(h r)$ & \multicolumn{2}{|c|}{$2[\mathrm{~T}]$} \\
\hline $\operatorname{MatC}(\$ / \mathrm{kg})$ & \multicolumn{2}{|c|}{110 avg. $^{6}$} \\
\hline Dens $\left(\mathrm{g} / \mathrm{cm}^{3}\right)$ & \multicolumn{2}{|c|}{0.71 avg. ${ }^{6}$} \\
\hline
\end{tabular}

In Table 4.2, the term ' $\mathrm{T}$ ' denotes a value that is typical of what you would find in the SLA process, though not directly quoted. The term ' $A$ ' denotes values that were approximated based on available information. Using the parameters in Table 4.2, build time and part cost were calculated for the SLS technologies.

\subsubsection{Fused Deposition Modeling (FDM)}

Unlike SLA and SLS, FDM is different in the fact that it does not use a laser to trace a cross-section. Instead, a heated thermoplastic material is extruded through a nozzle in the form of the model cross-section. The platform is maintained at a lower temperature to ensure the layer hardens quickly ${ }^{5}$. The platform then lowers and the nozzle deposits 
another material atop the previous. The process continues until a solid part is produced. The FDM system is manufactured by Stratasys.

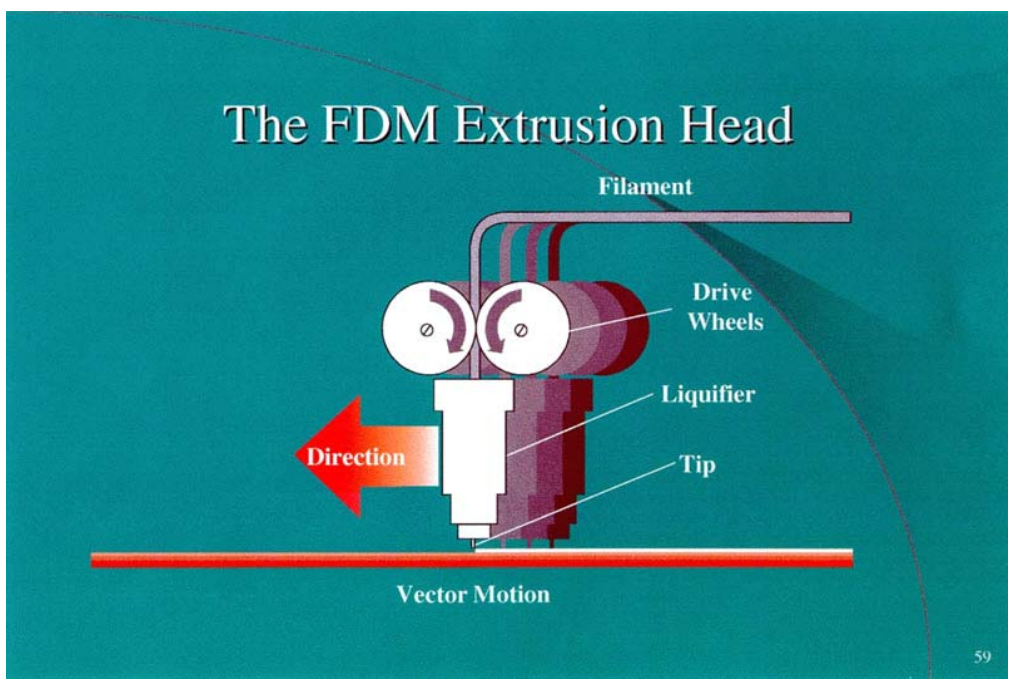

Figure 4. 8 Schematic of FDM process 5

The build time model for the FDM process is displayed in Figure 4.9.

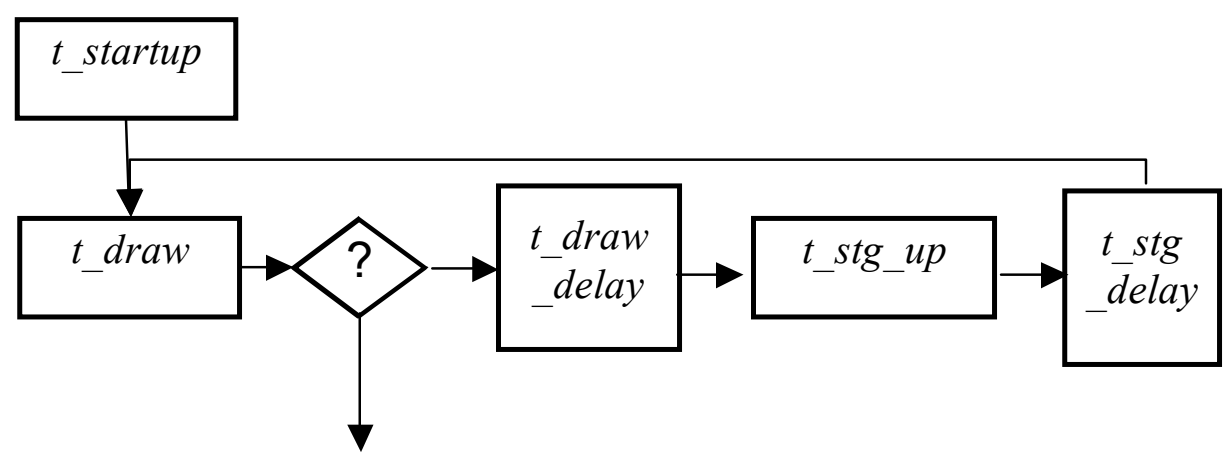

Figure 4. 9 FDM Build Time Model

Given the build time model in Figure 4.9, the parameters used for FDM build time and cost estimation are displayed in Table 4.3. The FDM machines considered in this thesis are the Prodigy Plus, Titan and Maxum machines, all by Stratasys. 
Table 4. 3 FDM Model Parameters

\begin{tabular}{|c|c|c|c|}
\hline & Prodigy Plus & Titan & Maxum \\
\hline L_vat $(\mathrm{mm})$ & 203 & 406 & 600 \\
\hline W_vat (mm) & 203 & 355 & 500 \\
\hline H_vat $(\mathrm{mm})$ & 305 & 406 & 600 \\
\hline $\operatorname{MachC}(\$)$ & 55 & 190 & 250 \\
\hline MaintC (\$) & \multicolumn{3}{|c|}{$0.1 * \operatorname{MachC}^{6}$} \\
\hline $\begin{array}{c}\text { Mach_rate } \\
(\$ / h r)\end{array}$ & \multicolumn{3}{|c|}{$25[\mathrm{~A}]$} \\
\hline tech rate $(\$ / h r)$ & \multicolumn{3}{|c|}{$10[\mathrm{~A}]$} \\
\hline Ul (yrs) & \multicolumn{3}{|c|}{$7[\mathrm{~A}]$} \\
\hline$N_{\text {mach }}$ & \multicolumn{3}{|c|}{$1[\mathrm{~A}]$} \\
\hline$D_{\text {scan }}(\mathrm{mm})$ & $0.61 \mathrm{avg}^{13}$ & $0.61^{13}$ & $0.579 \mathrm{avg}^{13}$ \\
\hline$V_{\text {scan }}(\mathrm{mm} / \mathrm{s})$ & 64 & 127 & 254 \\
\hline$V_{\text {jump }}(\mathrm{mm} / \mathrm{s})$ & 64 & 127 & 254 \\
\hline hatch & \multicolumn{3}{|c|}{$1[\mathrm{~T}]$} \\
\hline$t_{\text {laver }}(\mathrm{mm})$ & $0.25 \mathrm{avg}^{13}$ & 0.25 avg $^{13}$ & $0.25^{13}$ \\
\hline$N_{\text {scan }}$ & \multicolumn{3}{|c|}{$1[\mathrm{~T}]$} \\
\hline$N_{\text {scansupp }}$ & \multicolumn{3}{|c|}{$1[\mathrm{~T}]$} \\
\hline$z_{\text {supp }}(\mathrm{mm})$ & \multirow{2}{*}{\multicolumn{3}{|c|}{$\begin{array}{l}0[\mathrm{~T}] \\
0[\mathrm{~A}]\end{array}$}} \\
\hline supp factor & & & \\
\hline$t_{\text {draw delay }}(s)$ & \multicolumn{3}{|c|}{$2[\mathrm{~A}]$} \\
\hline$t_{s t g \text { down }}(s)$ & \multicolumn{3}{|c|}{0} \\
\hline$t_{\text {stg delav }}(s)$ & \multirow{2}{*}{\multicolumn{3}{|c|}{$\frac{2[\mathrm{~A}]}{0}$}} \\
\hline$t_{\text {sweep }}(s)$ & & & \\
\hline$t_{s t g}$ up $(s)$ & \multicolumn{3}{|c|}{$10[\mathrm{~A}]$} \\
\hline$t_{\text {swp delav }}(s)$ & \multicolumn{3}{|c|}{0} \\
\hline$t_{\text {preproc }}(h r)$ & \multicolumn{3}{|c|}{$1[\mathrm{~T}]$} \\
\hline$t_{\text {prostproc }}(\mathrm{hr})$ & \multirow{2}{*}{\multicolumn{3}{|c|}{$\frac{1[\mathrm{~T}]}{0.5[\mathrm{~T}]}$}} \\
\hline$t_{\text {startup }}(h r)$ & & & \\
\hline $\operatorname{MatC}(\$ / \mathrm{kg})$ & \multicolumn{3}{|c|}{250 avg. ${ }^{6}$} \\
\hline Dens $\left(\mathrm{g} / \mathrm{cm}^{3}\right)$ & \multicolumn{3}{|c|}{1 avg [T] } \\
\hline
\end{tabular}

In Table 4.3, the term ' $\mathrm{T}$ ' denotes a value that is typical of what you would find in the FDM process, though not directly quoted. The term 'A' denotes values that were approximated based on available information. Using the parameters in Table 4.3, build time and part cost were calculated for the FDM technologies.

\subsection{MATLAB GUI TOOL}

\subsubsection{Description}

For the build time and cost estimation methods described in the earlier sections, a Graphical User Interface (GUI) was developed to aid the designer in determining these selection attributes. The GUI will function as displayed in Figure 4.10, where the user 
will input the part geometry and RM build characteristics. Using the machine characteristics encoded in the software, the build time and part cost are computed.

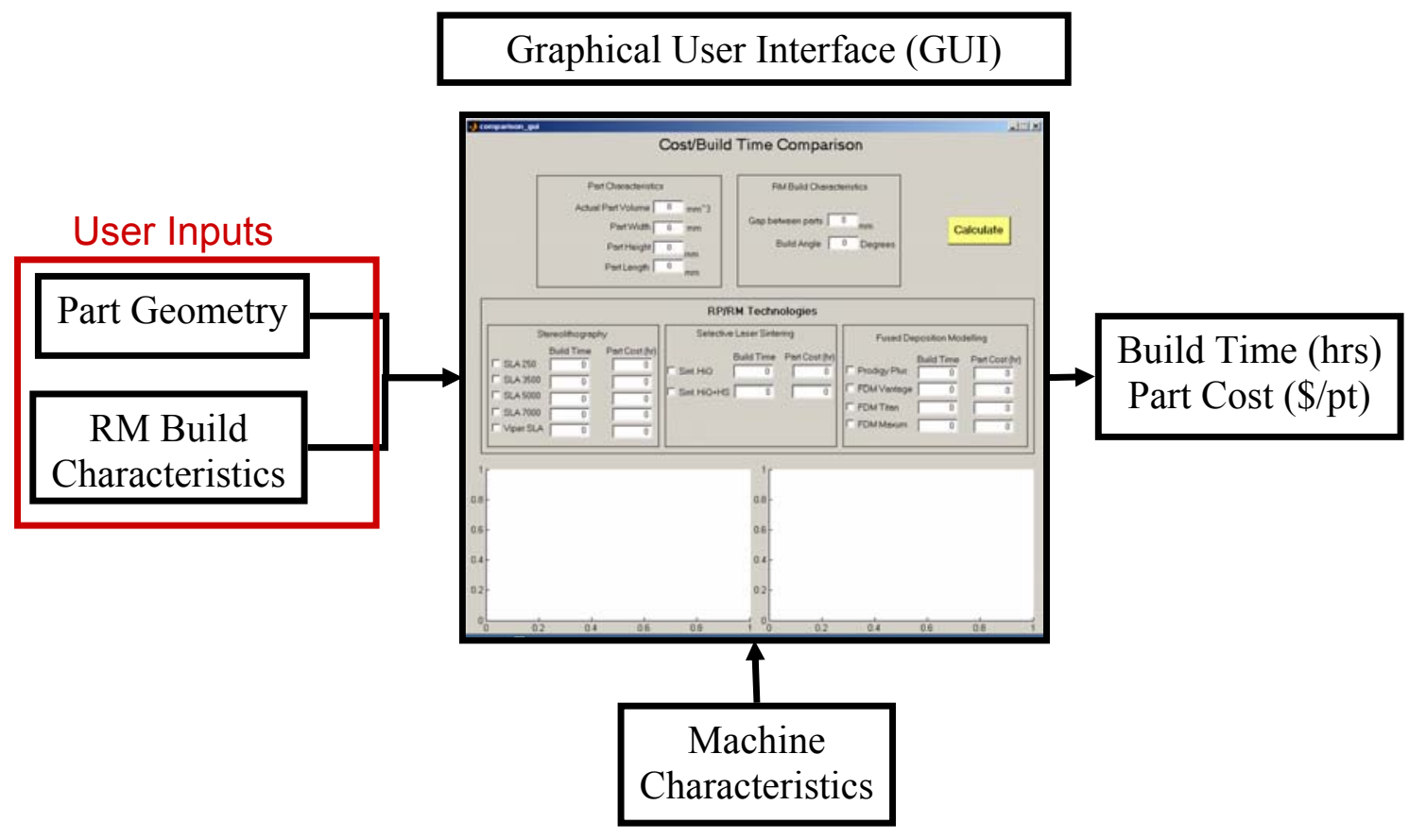

Figure 4. 10 GUI diagram

The GUI was developed in Matlab v6.5 using the 'guide' function. A screenshot of the GUI is displayed in the Figure 4.11. 


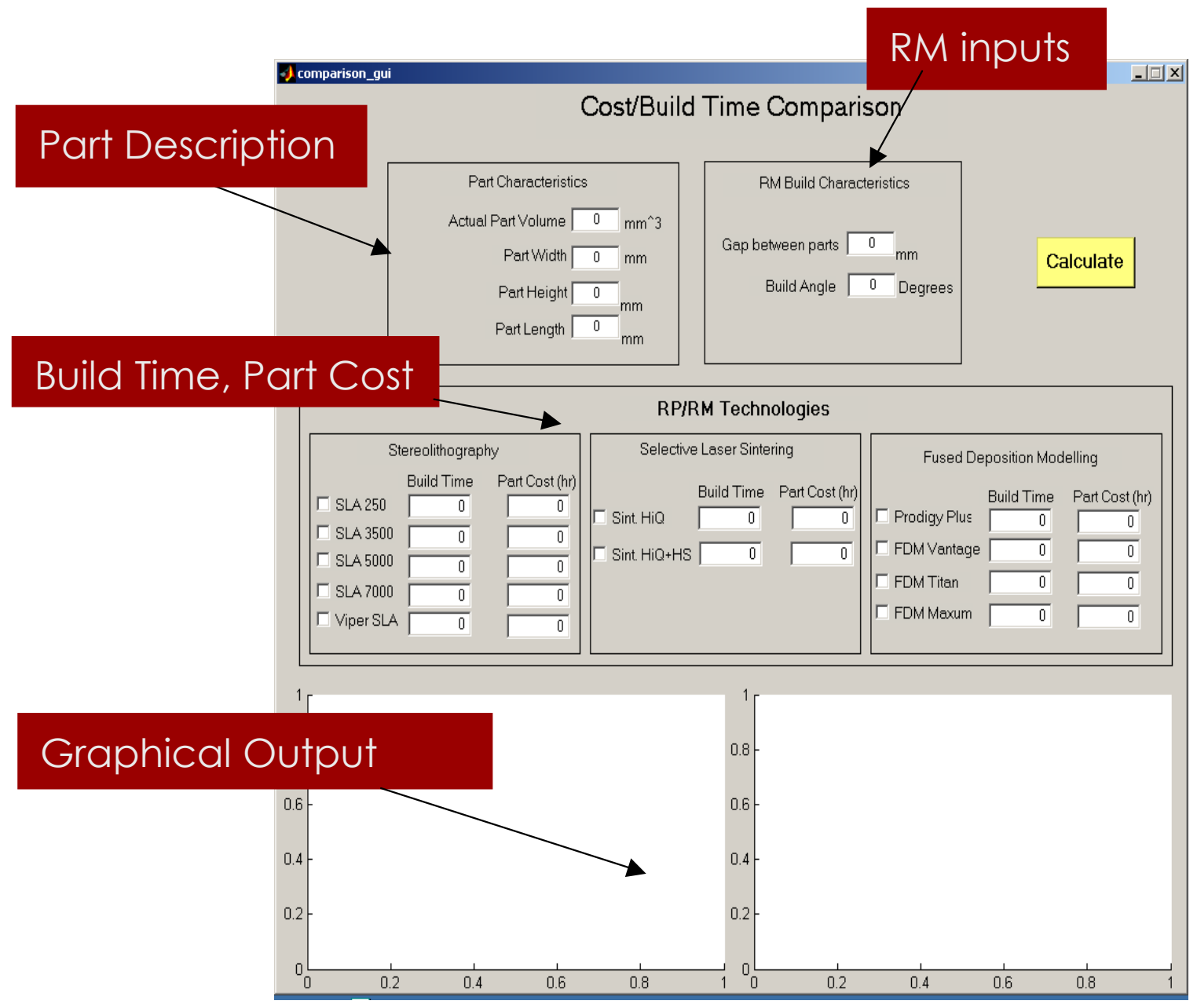

Figure 4. 11 Screenshot of GUI

There are 4 main sections to the GUI displayed in Figure 4.11: part description, RM inputs, build time and part cost outputs, and a graphical outputs. These sections are described in detail in the following sections.

\subsubsection{Part Description}

The part description section of the GUI will input the geometric characteristics of the part. These inputs include the actual part volume (which can be either estimated using various mathematical methods or determined using CAD software), part width, part height, and part length. The part width, height, and length are used to determine the bounding box of the part, thus should be the maximum values. This bounding box, as 
described earlier, will be used to determine the number of part per build, as well as the area factor $(f(\rho))$.

\subsubsection{RM Build Characteristics}

This section of the GUI is where the user will input the characteristics of the build. Since we are considering Rapid Manufacturing for these estimations, part spacing, as well as build angle are important aspects to consider. Part spacing (gap between parts) is the spacing between the parts in the vat. The build angle is used to determine the orientation of the part. For builds where surface finish and part accuracy are important, the user can determine the build times and costs for building these parts at different angles to maximize the aforementioned characteristics.

\subsubsection{Build Time and Part Cost Outputs}

This section of the GUI serves a dual purpose: select RM technologies as well as to output numerical values. The GUI will output numerical values for build time (hours) and part cost (\$). The user will select the RM technologies by checking the box next to the respective technologies. Numerical values will only be output for the selected technologies. By listing and characterizing different machines within a given technology, this allows the user the flexibility to compare within specific technologies or across general technologies.

\subsubsection{Graphical Output}

This section of the GUI will output a graphical display for the user. The display will be a bar chart of the numerical values displayed in the above section.

\subsection{TESTING THE VALIDITY OF THE BUILD TIME AND COST ESTIMATION METHODS}

To check the validity of the build time and cost estimation technique, some examples were done and the results analyzed. In Section 4.5.1, two parts with equal cross-sectional areas, but different geometries, were compared in an attempt to validate the use of 
Pham's correction factor. The build times from the Build Time Estimator (BTE) were quantitatively compared to test data in Sections 4.5.2 and 4.5.3. In Section 4.5.2, the SLS-specific build time model was evaluated and in Section 4.5.3, the SLA-specific build time model was evaluated using single and multiple part comparisons. In Section 4.5.4, the Build Time and Cost Estimator were run on a test part and the results qualitatively compared.

\subsubsection{Correction Factor Comparison.}

For the first example, the values for two test parts were input into the BTE to compare the results obtained when the area factor ${ }^{38}$ was used. For this example, the 2 parts have the same cross-sectional area and volume, but different geometries. The two cross-sections are displayed in the Figure 4.12.
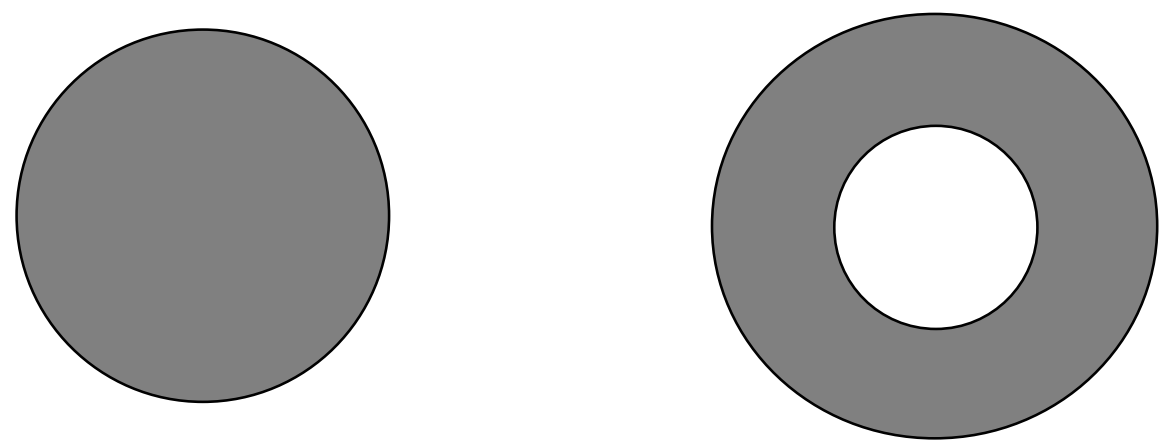

Figure 4. 12 Cross Sections for Example 1

First, the build time was calculated for the first shape without using any sort of correction factor. Next, the build times for the two parts were calculated using the Pham's correction factor. These results are compared in Table 4.4. 
Table 4. 4 Build Time Results for Pham's factor comparison

\begin{tabular}{|l|l|l|l|l|}
\hline & Dimensions & Average Area & $\rho$ & Build Time \\
\hline Baseline & $\begin{array}{l}\text { Dia: } 50.8 \mathrm{~mm} \\
\text { H: } 50.8 \mathrm{~mm}\end{array}$ & $2026.83 \mathrm{~mm}^{2}$ & & 7.399 hrs. \\
\hline $\begin{array}{l}\text { Part 1 } \\
\text { (using CF) }\end{array}$ & $\begin{array}{l}\text { Dia: } 50.8 \mathrm{~mm} \\
\text { H: } 50.8 \mathrm{~mm}\end{array}$ & $2512.0 \mathrm{~mm}^{2}$ & 0.7854 & $7.64 \mathrm{hrs}$. \\
\hline $\begin{array}{l}\text { Part 2 } \\
\text { (using CF) }\end{array}$ & $\begin{array}{l}\text { O_Dia: } 56.796 \\
\text { mm }\end{array}$ & $2939.2 \mathrm{~mm}^{2}$ & 0.6283 & $7.852 \mathrm{hrs.}$ \\
& $\begin{array}{l}\text { I_Dia: } 25.4 \mathrm{~mm} \\
\text { H: } 50.8 \mathrm{~mm}\end{array}$ & & & \\
\hline
\end{tabular}

As displayed in the table, the build times calculated with Pham's factor are higher than that without the factor. This is because this correction method not only accounts for the time in which the laser is scanning, but also when the laser is jumping either across the cross-section or to the next part. As can be seen in Table 4.4, the build time increases slightly as the amount of empty space within the cross-section increases. It should be noted that the space around the circular cross section defined by a bounding box around its extremities is also considered empty space.

\subsubsection{Quantitative Evaluation of the Build Time Estimator using SLS}

In this example, the validity of the BTE for SLS was tested using actual test data from Pham et al.'s ${ }^{38}$ paper. As in Pham's paper, trials were run using SLS and RapidSteel2 and Protoform as materials. The BTE was run on the SLS Sinterstation HiQ with the conditions found in Table 4.5. These conditions mimic those that are found in the paper.

Table 4. 5 Build time Part and Machine Characteristics

\begin{tabular}{|r|c|c|}
\hline Parameter & Trial 1 (RapidSteel2) & Trial 2 (Protoform) \\
\hline Vol_act $\left(\mathrm{mm}^{3}\right)$ & 604430 & 2974.72 \\
\hline Part_height $(\mathrm{mm})$ & 40 & 64 \\
\hline D_scan $(\mathrm{mm})$ & 0.08 & 0.152 \\
\hline Number of parts & 1 & 3 \\
\hline Vol_bb $\left(\mathrm{mm}^{3}\right)$ & 888867 & 35840 \\
\hline
\end{tabular}

The results from the trials are displayed in Table 4.6. From the paper, actual build times are also listed and compared. 
Table 4. 6 Results from Example 2

\begin{tabular}{|r|c|c|c|}
\hline Trial (Material) & Predicted Time $(\mathrm{h})$ & Actual Time $(\mathrm{h})$ & Error $(\%)$ \\
\hline 1 (RapidSteel2) & 33.15 & 31.2 & 6.3 \\
\hline 2 (Protoform) & 4.94 & 5.5 & 10.1 \\
\hline
\end{tabular}

As displayed in the table above, the BTE came within 6.3\% (for Trial 1) and $10.1 \%$ (for Trial 2). These errors are within the typical range for most build time estimators that I have seen. It shows (at least for these trials) that as the cross-sections and volumes of part increase, the error for the BTE decreases.

\subsubsection{Quantitative Evaluation of the Build Time Estimator using SLA}

In this section, the SLA-specific build time model was evaluated using single (SLA 3500) and multiple part builds (SLA Viper). In both cases, the BTE was compared to data collected using the Buildstation (v5.4 by 3DSystems) SLA build time estimation software and the actual build time of the parts.

\subsubsection{Single Part BTE Evaluation}

The mold insert model, displayed in Figure 4.13, was used for the single part build time comparison. In this example, the SLA 3500 RP machine was used.

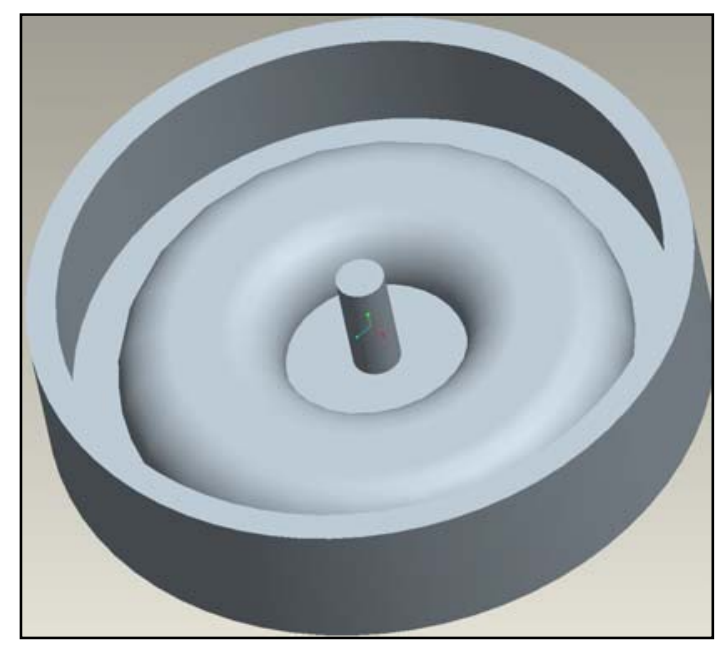

Figure 4. 13 Mold Insert model 
Using the model in Figure 4.13, the build time for a mold insert was determined using 3 methods: using the parametric BTE, using Buildstation software, and actual build time using SLA 3500 RP machine by 3D systems. The two estimated build times and the actual build time are displayed in the following table.

Table 4. 7 Estimated and Actual build time comparison

\begin{tabular}{|l|c|c|}
\hline \multicolumn{1}{|c|}{ Method } & $\begin{array}{c}\text { Build Time } \\
\text { (hours:mins) }\end{array}$ & $\begin{array}{c}\text { Relative } \\
\text { Error (\%) }\end{array}$ \\
\hline Parametric BTE & $3: 10$ & 2.2 \\
\hline Build station & $2: 49$ & 8.3 \\
\hline Actual build time & $3: 03$ & \\
\hline
\end{tabular}

As illustrated in Table 4.7, the build time estimated using the parametric BTE was found to be more accurate than that using exact geometry. It should be noted that the use of the empirically determined factor, $\alpha$, allows for some adjustment of the build time based on the characteristics of the specific technology used. For this case, a value of 0.5 was used. It should be noted that even if the approximated build time was found to be inaccurate, it would provide a constant means of estimating the build time. Therefore, it still could be used as a criterion upon which to select a RM technology under uncertainty.

\subsubsection{Multiple Part BTE Evaluation}

Since the build time and cost models in this thesis will be used for RM, we must also evaluate the models with respect multiple parts. For this evaluation, the BTE was also compared to that of Buildstation and to the actual build time (simulated) in the SLA Viper machine. For calculation of the actual build time, several trials of multiple parts were run and compared to the results from Buildstation. In this evaluation, it was determined that Buildstation underestimated the actual build time by an average of approximately $10 \%$. Since this evaluation included a large number of test trials, the actual build time was estimated based on a correction factor of $10 \%$ from the Buildstation estimate, or Buildstation*1.1 = Actual Build Time. 
For this evaluation, two test parts, displayed in Figure 4.14 and 4.15, were used. These parts differ in geometry and material distribution, therefore giving us two exclusive case studies.

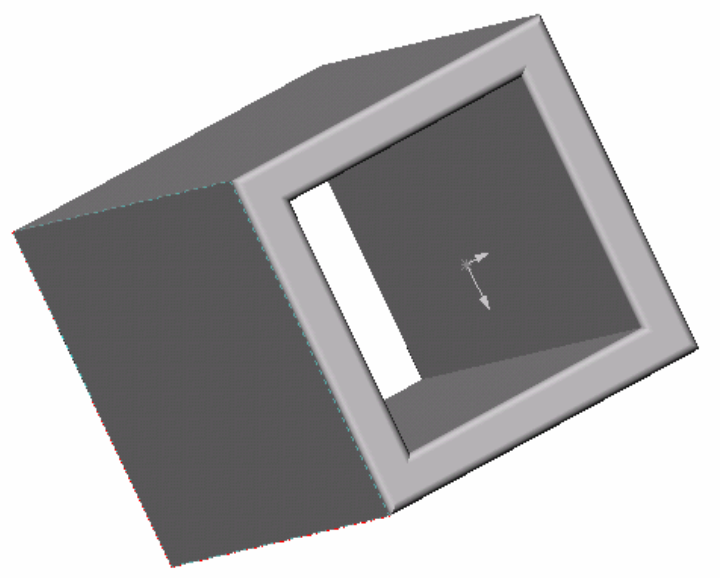

Figure 4. 14 Part 1 for multiple part evaluation

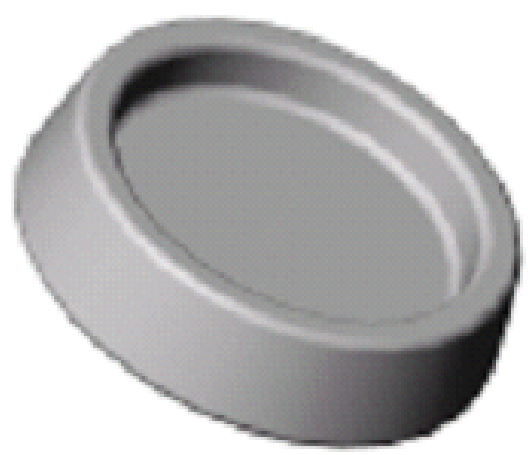

Figure 4. 15 Part 2 for multiple part evaluation

For the evaluation, the volume (by way of height and material distribution) of each part was varied and the build times calculated and compared. The build times are compared in Figures 4.16 and 4.17. 


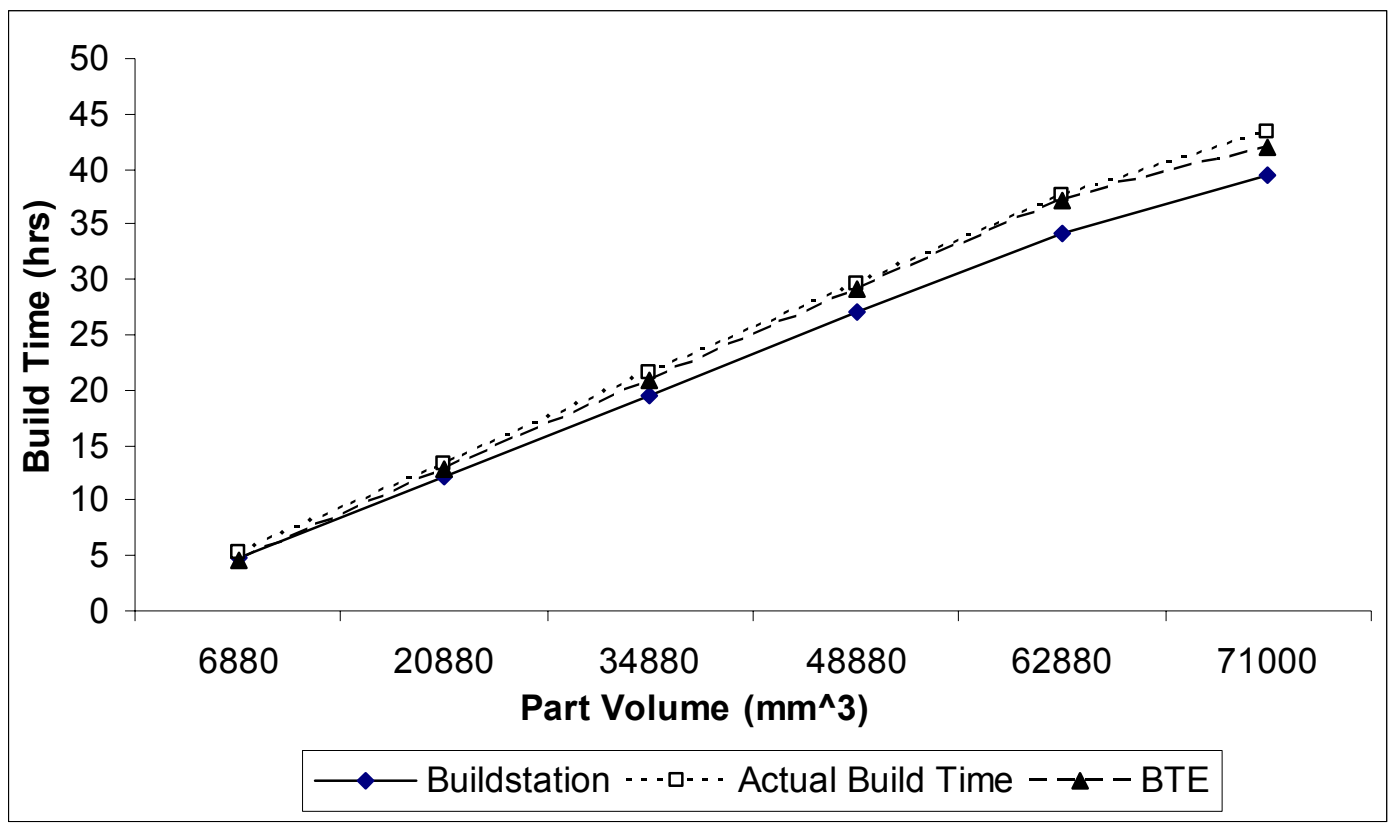

Figure 4. 16 Build Time Comparison for Part 1

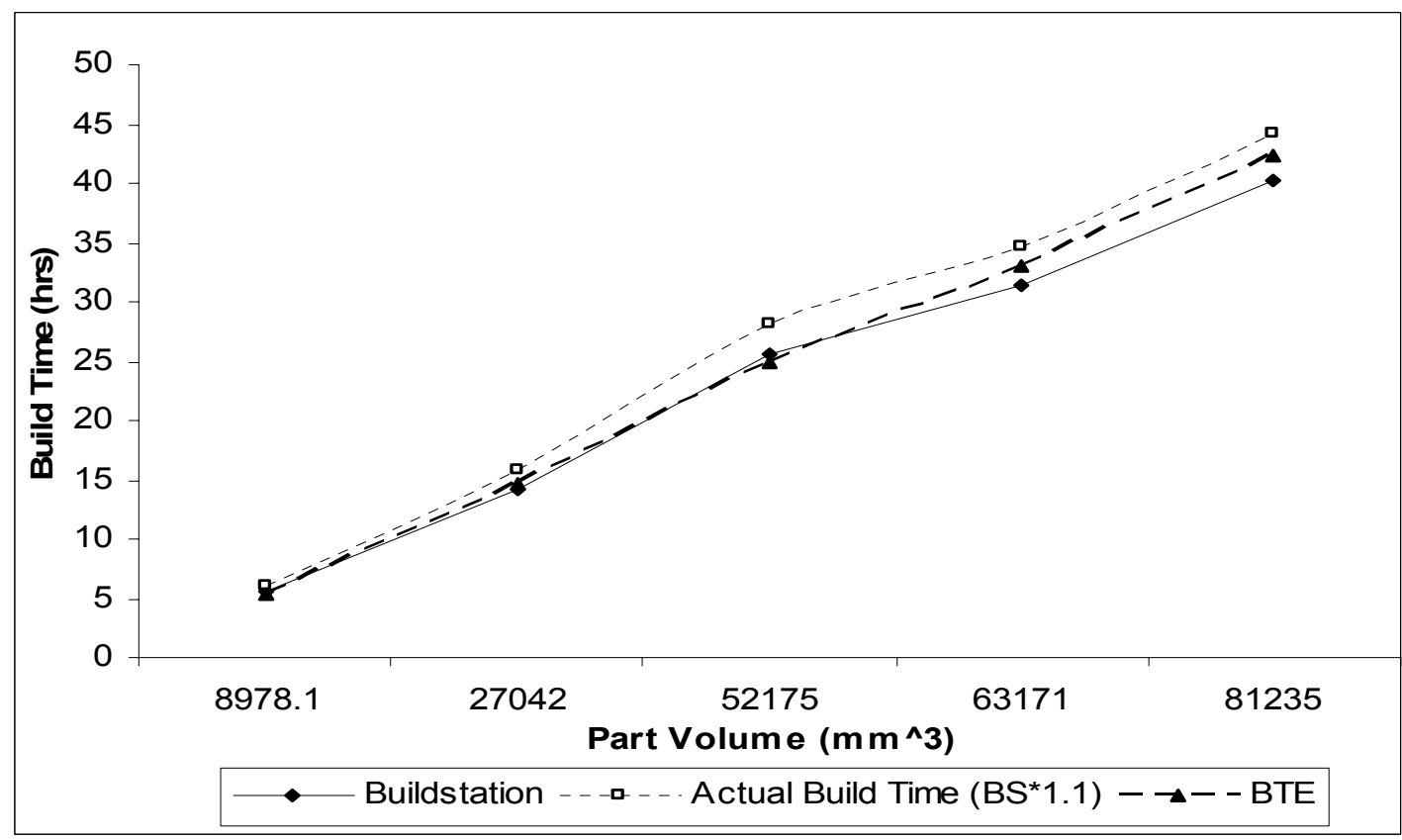

Figure 4. 17 Build Time Comparison for Part 2

As seen in Figures 4.16 and 4.17, the build time calculated from the BTE compares very well to that of the actual build time. In both cases, the BTE estimate gave a better 
average approximation of the actual build time than the Buildstation software. As explained in Section 4.2, the build time is calculated based on three constituent terms: draw time, delay time, and startup time. In order to better characterize the BTE, these individual terms were also plotted against the part volume in Figures 4.18 and 4.19.

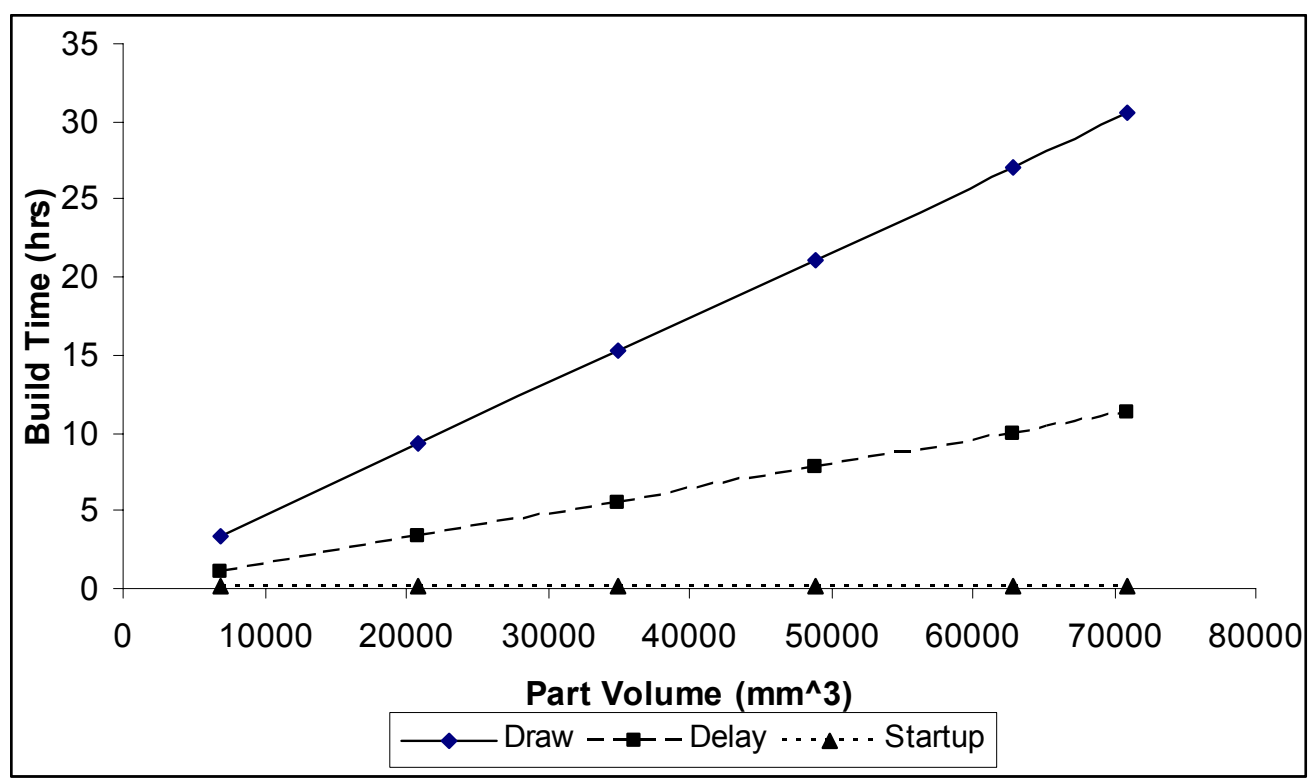

Figure 4. 18 BTE Individual Terms for Part 1

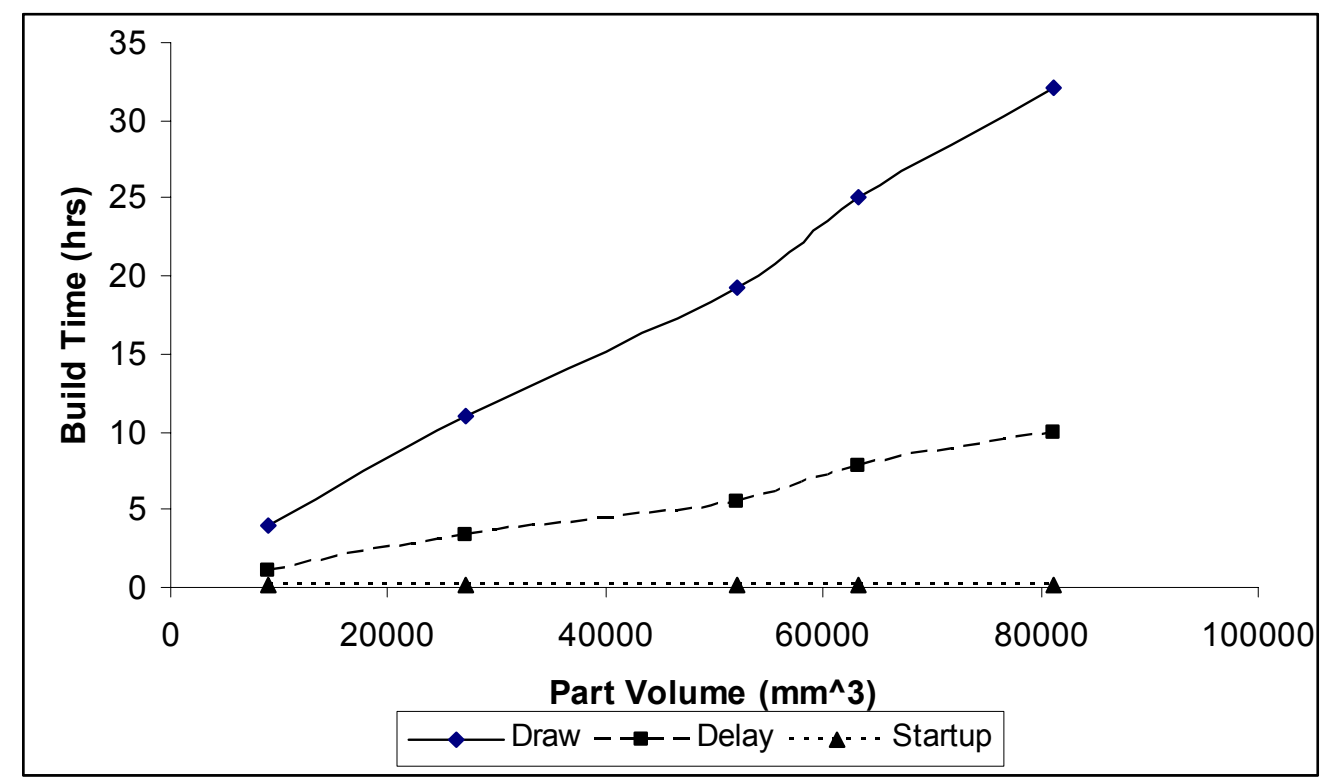

Figure 4. 19 BTE Individual terms for Part 2 
For both parts in Figures 4.18 and 4.19, the same general trends can be seen. In both cases, the draw time increases at a much faster rate than that of the delay time and startup time (which is constant across the volume increase). From these trends, we can see that as the volume increases, the draw time becomes an increasingly larger contributor to the total build time of the parts. At smaller volumes, the time between builds (delay time) is a significant factor in the total build time of the part. As the volume increases, these delay times, which depends mainly on part height, become a smaller contributor to the total build time. This can be seen in Figure 4.20, where the height of the part is kept constant and the volume increased.

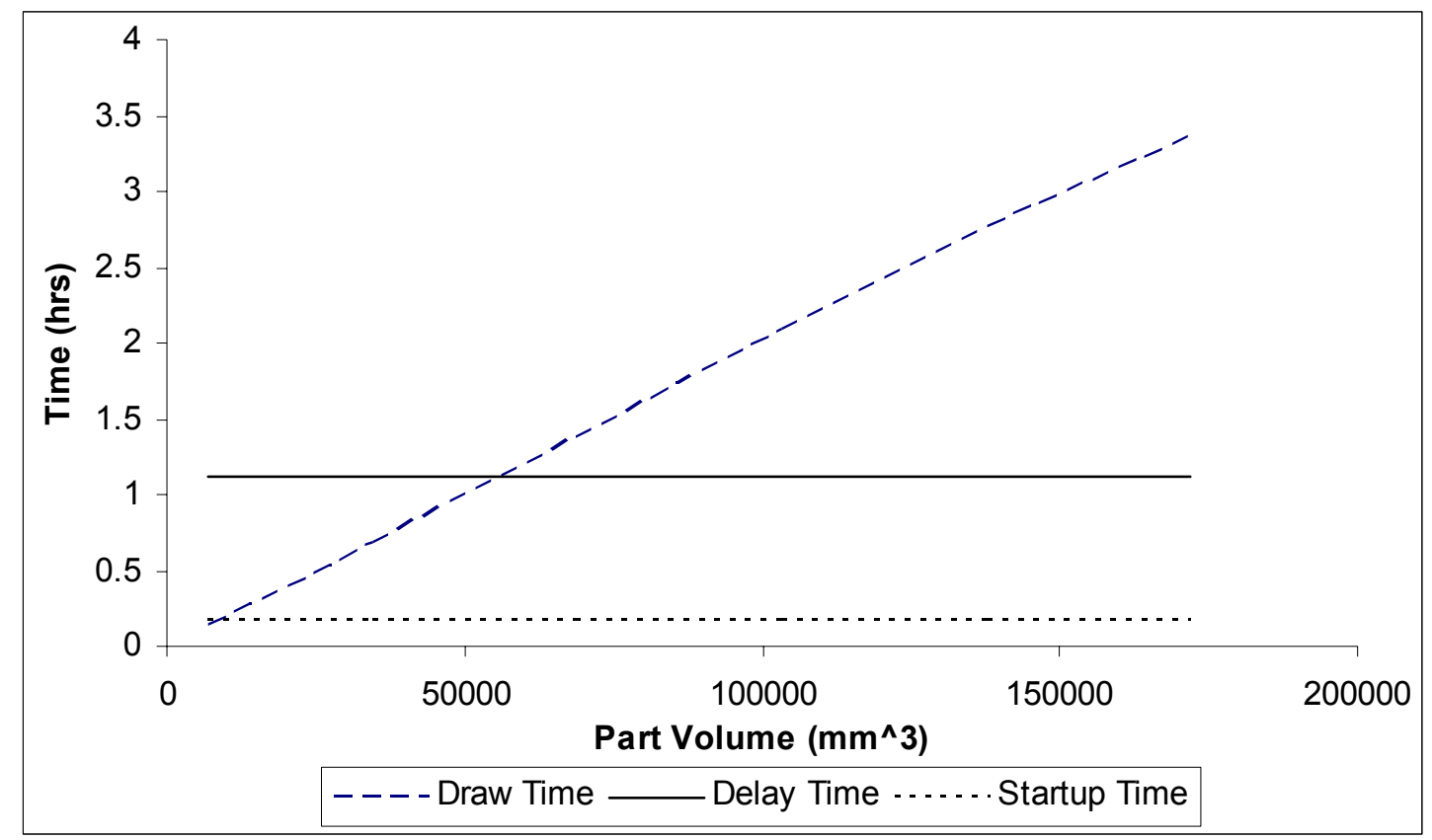

Figure 4. 20 BTE comparison (constant height)

When considering RM, we are considering a large number of parts being built in a single build, or a full vat. In this case, we are dealing with large volumes being built. At this point, as stated earlier, the draw time is the major contributor to the total build time. Therefore, the accuracy of the BTE will depend largely on the accuracy of the draw time calculation. 
The draw time, using Eq. 4.3, is calculated as a function of the scan distance and scan velocity. In our build time model, the scan distance depends largely on the part geometry (average cross-sectional area) and machine characteristics (scan diameter and hatch spacing), where the scan velocity depends only on the machine characteristics. In the evaluation, the scan velocity is the only parameter of interest since the scan distance and its constituent values are deterministic and certain. On the other hand, with scan velocity, the accuracy of these values is variable and uncertain, for most cases.

In a sensitivity study, a change in the scan velocity of $10 \%$ yielded a $4.5 \%$ change in the build time calculated using the BTE. Because of the uncertainty involved in the calculation of the scan velocity, these values are sometimes reported with ranges of almost $200 \%$. Because of this, there is a limitation to the accuracy of the BTE. The accuracy of the BTE will depend largely on the accuracy of the values used for scan velocity.

The part cost was also estimated for parts 1 and 2 and is displayed in Figures 4.21 and 4.22 .

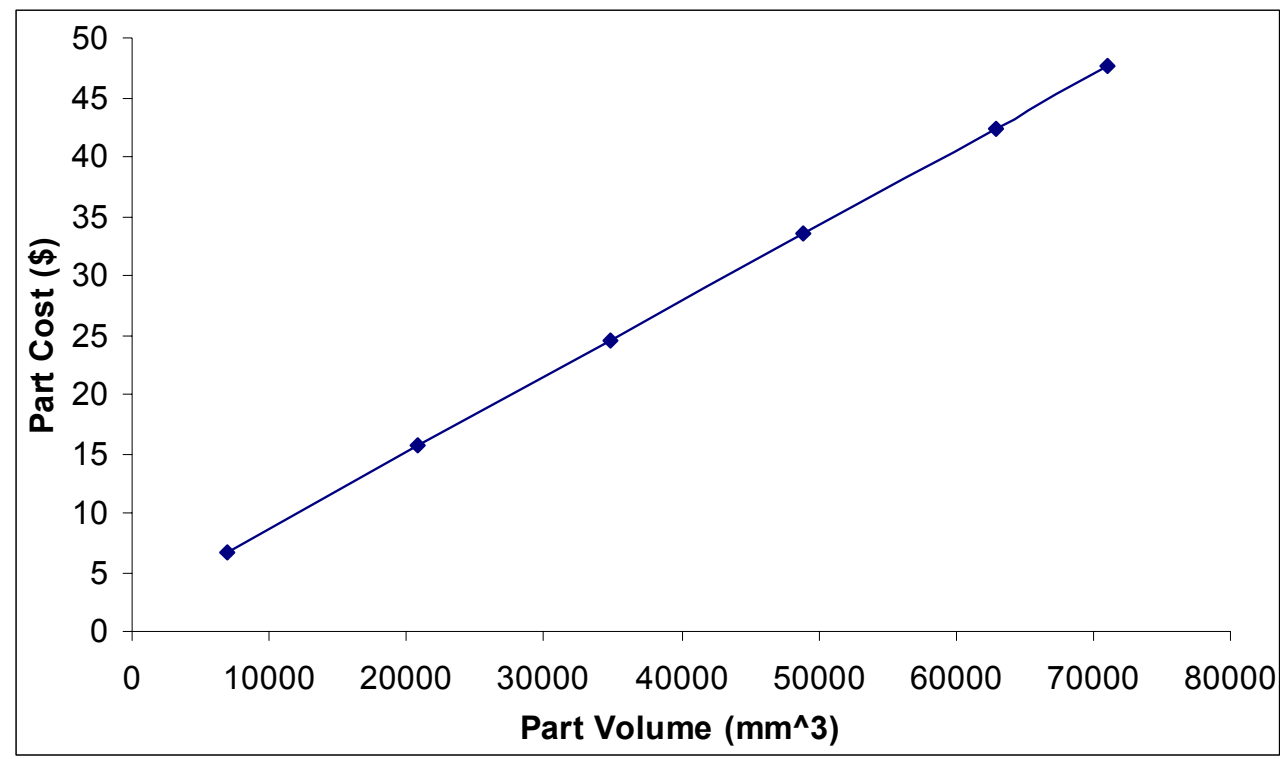

Figure 4. 21 Part Cost Estimation for Part 1 


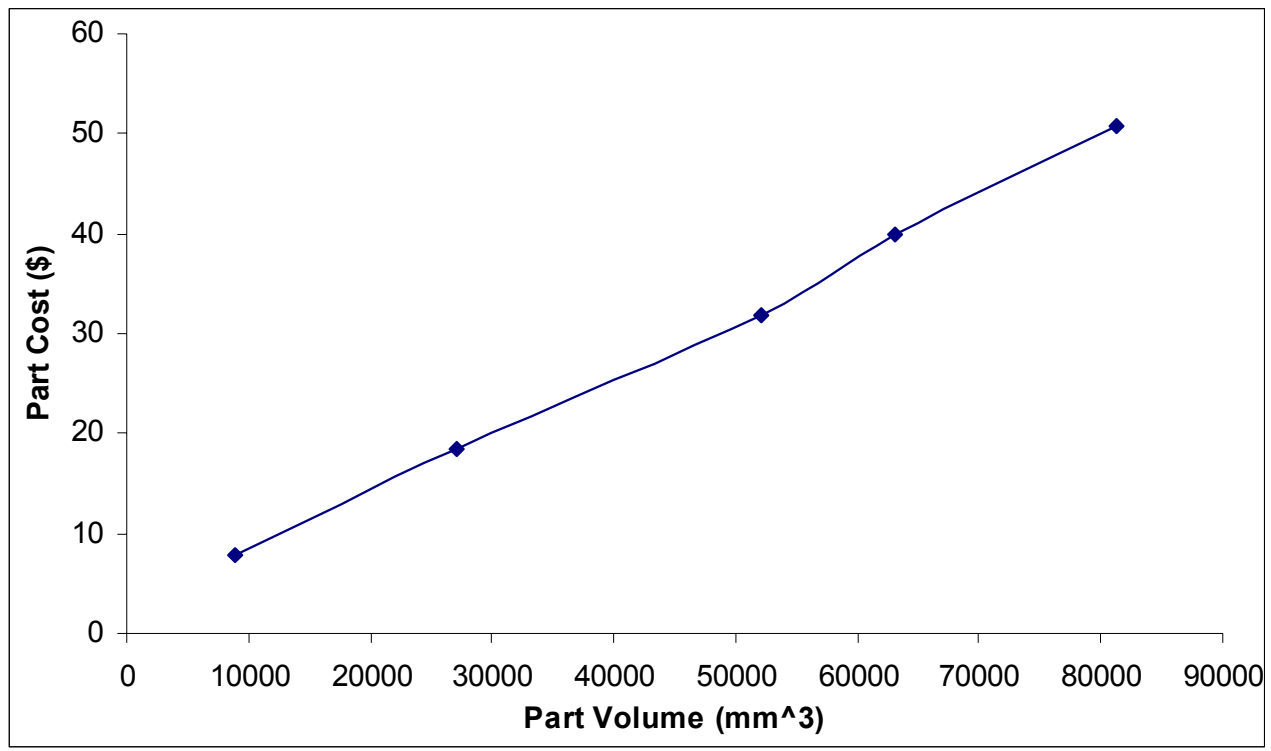

Figure 4. 22 Part Cost Estimation for Part 2

As displayed in Figures 4.21 and 4.22, for both cases, the part cost increases as the part volume increases. Although this general trend seems in order, more evaluation is needed to improve the validity of the part cost estimation model. As explained in Section 4.3, the part cost is calculated based on 4 constituent terms: material cost, maintenance cost, machine cost, and operation cost. The relationship between these individual terms and part volume are evaluated in Figures 4.23 and 4.24. 


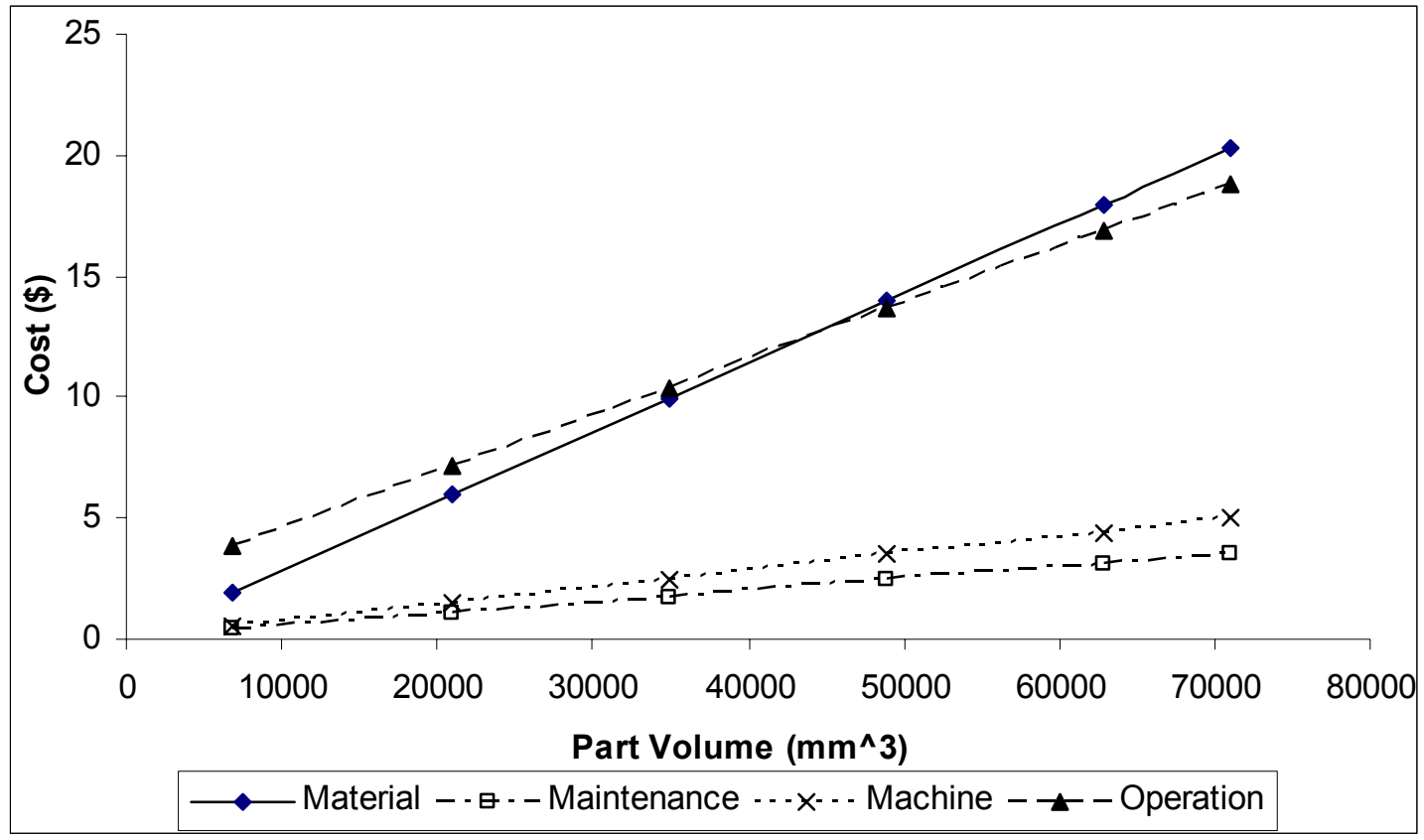

Figure 4. 23 Individual cost terms versus part volume for Part 1

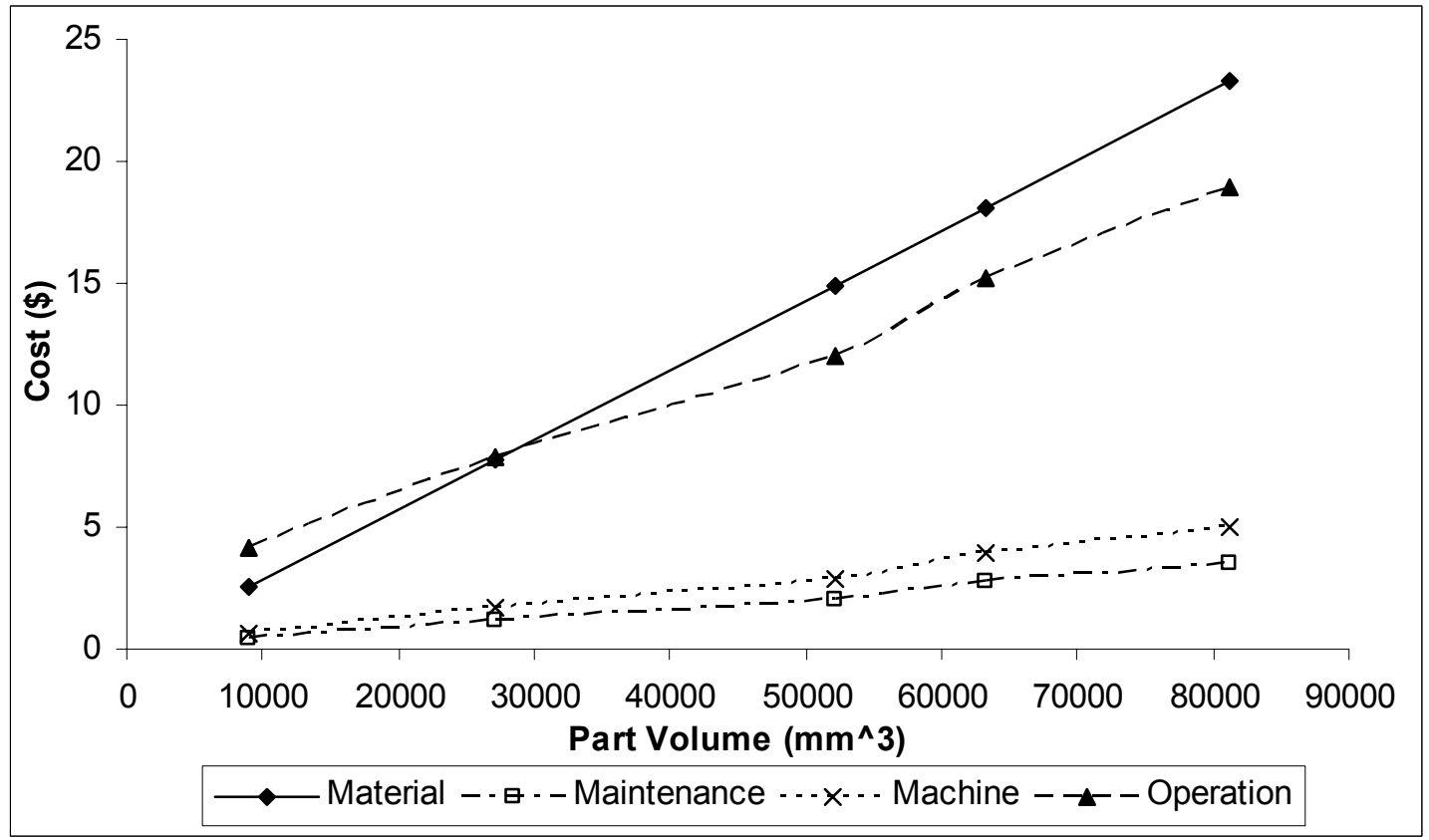

Figure 4. 24 Individual cost terms versus part volume for Part 2

As seen in Figures 4.23 and 4.24, with respect to volume increase, the material costs and operation costs increase at a faster rate than that of the maintenance costs and machine costs. This is due to the fact that material cost (Eq. 4.11) is primarily dependent on part 
volume, and operation cost (Eq. 4.14) is dependent on the build time (which is based on volume) and pre- and post-processing times of the RM process. The main source of uncertainty in these values is the machine rate, which is the hourly rate associated with running the RP machines. From a sensitivity analysis, a $10 \%$ change in the machine rate yielded a $2.4 \%$ change in the part cost. Because of the uncertainty in the value for machine rate, further study must be performed to reduce the uncertainty associated with this value. However, a $2.4 \%$ change can be considered insignificant, given the potentially small range that can be assumed for machine rate. The maintenance and machine costs increase at a much slower rate than that of the material cost and operation cost.

\subsubsection{Qualitative Comparison of Build Time and Cost}

In this comparison, the total Build Time and Cost Estimator were qualitatively evaluated.

For this example, a sample part was built and the results compared. The inputs and outputs of the Estimator are displayed in Figure 4.25. 


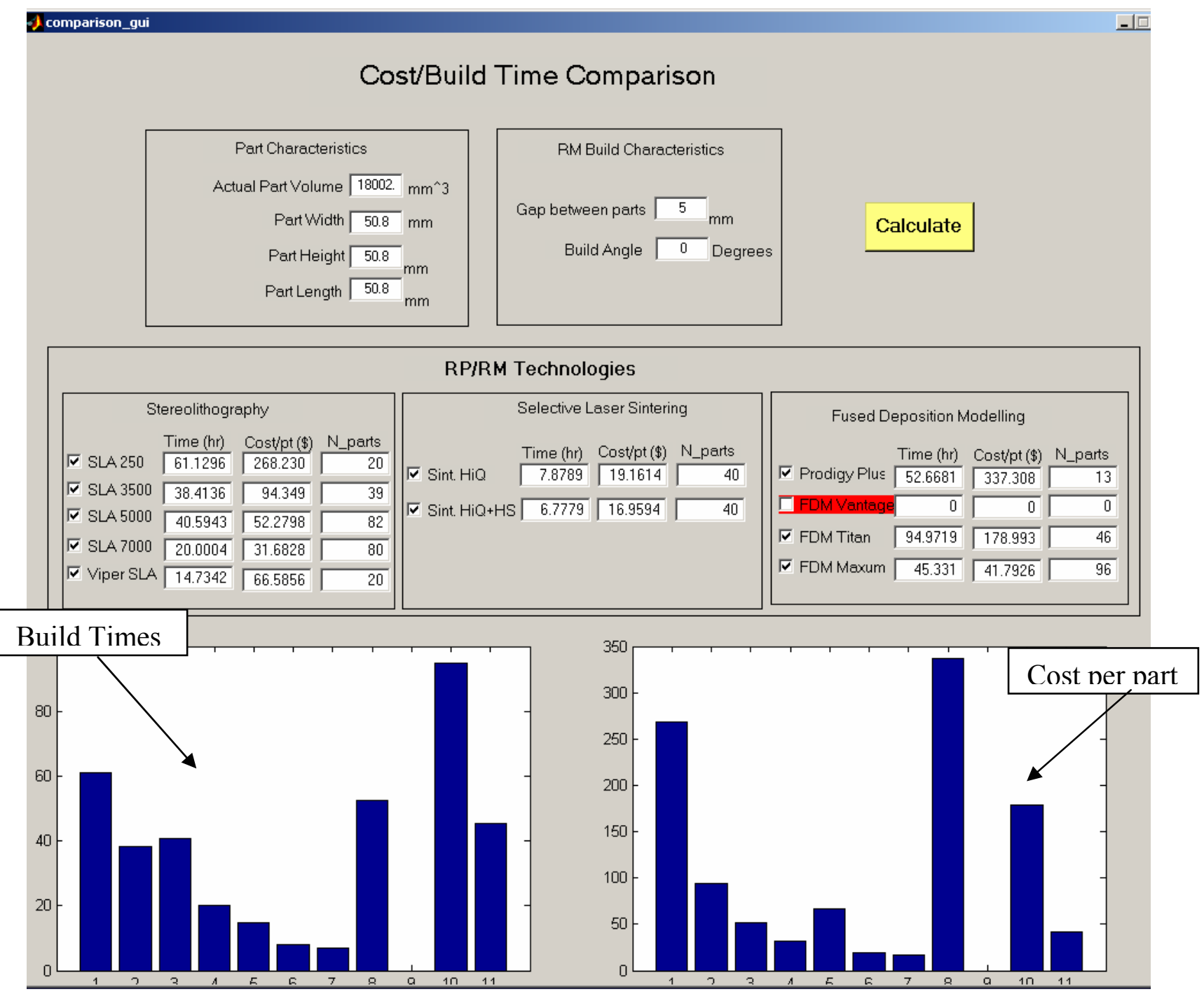

Figure 4. 25 Screenshot of Inputs and Outputs for qualitative comparison

As you can see in the figure above, the results seem typical of what you would see in an actual build comparison. It should be noted that when comparing these technologies, because we are focused on Rapid Manufacturing, the number of parts produced in a build must also be taken into account. When looking within Stereolithography technologies, build time is downward sloping as the technology, and expense of the technology, increases. Cost per part also decreases, except for the Viper because of its small build chamber. When looking at Selective Laser Sintering, the Sinterstation HiQ+HS produces a faster build time (and lower cost per part) because of the high speed laser that was added. For Fused Deposition Modelling, the FDM Maxum showed the fastest build time 
and cost per part, which would be expected because of the reported $50 \%$ faster build times by Stratasys. Although the build time for the FDM Titan was much higher than that of the Prodigy Plus, the Titan produced 46 parts, compared with 13 for the Prodigy Plus.

\subsection{ADVANTAGES/ LIMITATIONS OF BUILD TIME AND PART COST ESTIMATION METHODS}

The build time and cost estimation models presented in Section 4.2 and 4.3 have several advantages in the field of RM. In this thesis, we have introduced the notion of technology investment for production of customized products. In other words, we are selecting a RM technology to be used across a range of products, over a period of time. In industry, build time and part cost estimation software available require the use of a CAD model to estimate the build time of parts. With the BTE model presented in this thesis, only overall geometry (bounding box and volume) is needed.

Another advantage to using the build time and part cost estimation models is that they can be expanded for use with uncertainty, as this thesis deals with. Since the models are simple and computationally inexpensive, uncertainty can be entered and propagated through the calculations. This is valuable when dealing with cases of uncertain geometric parameters.

The third advantage to using these models is that they are parametric, meaning that its solution depends only on the values input for the parameters. These universal models can be specified to account for any RP machine available, as long as the machine-specific information is available. By using the same general model to estimate these attributes, a common ground for comparison can be established.

However, the use of these models does not come without limitation. The main limitation to these models is that since they are parametric, they are only as accurate as the information used to characterize the process. In a perfect world, information about the 
machine characteristics will be limitless. However, when dealing with proprietary information, this information is not readily assessable and there exists a need for estimation and assumption. These estimations and assumptions reduce the accuracy of the build time and part cost estimation models.

\subsection{CHAPTER SUMMARY AND VALIDATION}

The role of Chapter 4 was to present and validate the build time and cost estimation models used to support the RM selection process. In Section 4.1, the overall RP build process was presented. In the context of this build process, the general parametric build time and cost models were presented in Sections 4.2 and 4.3. Depending on the parameters used, these models can be further specified for the respective RP technologies. The technology-specific models for SLA, SLS, and FDM were presented in Section 4.4 and the Matalb GUI tool used to evaluate the models was presented in Section 4.5. In Sections 4.6 and 4.7, the build time and cost estimation models were evaluated and their advantages and limitations explored.

The purpose of this chapter was to also verify that part cost and build time can be quantified for RM technologies with limited geometric information due to customization (Research Question \#3). The parametric build time and part cost models presented in this chapter depend explicitly on the parameters that characterize each technology and the overall part characteristics. Because of this dependence, the accuracy of the build time and part cost estimations depends on the accuracy of the parameters used in the models. In Section 3.4, sensitivity analyses were performed to see which parameters significantly affect the models. Also, the results from the models were compared to software estimations and actual build times of selected parts. It was concluded that even though the parametric build time and cost models only utilizes limited geometric information about the part (bounding box and part volume), the models still provide estimations that compare favorably to methods that require the use of a CAD model. 
In Chapter 5, illustrative examples will be presented to support the use of the selection method presented in this thesis. 


\section{CHAPTER 5 ILLUSTRATIVE EXAMPLES}

Mass customization is the production of custom, individually designed products at relatively low cost. One of the major enabling technologies of mass customization is Rapid Manufacturing (RM). RM is the use of Rapid Prototyping (RP) technologies to manufacture end-use products. The customization ability of RM introduces a considerable amount of uncertainty to the design process. This uncertainty must be considered in the selection process. In Chapter 3, the Selection for Rapid Manufacturing selection method was introduced. In this chapter, the author will provide illustrative examples of the use of this method. In Section 5.1, an example of the direct production of caster wheels is presented. In Section 5.2, an example of the production of hearing aid shells is presented. The results of these selection processes will be compared to a traditional selection method where uncertainty is not considered. RAMAS ${ }^{\circledR}$ RiskCalc 4.0 risk assessment software was used to propagate the uncertainty in the example problems.

\subsection{ILLUSTRATIVE EXAMPLE: DIRECT PRODUCTION OF CASTER WHEELS}

In this section, we consider the selection of a RM technology for Albion, Inc. Albion is one of the world's leaders in the engineering and production of institutional and industrial casters and wheels. A model of a typical caster wheel is displayed in Figure 5.1.

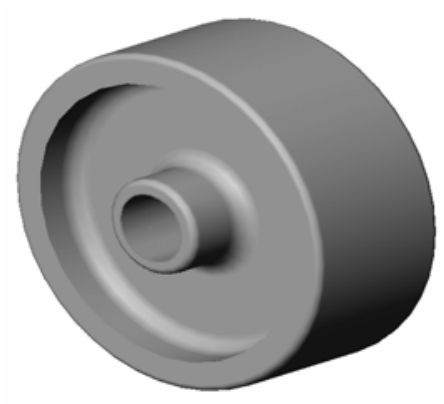

Figure 5. 1 Model of Caster Wheel 
Albion is known for offering a broad range of products for various 'typical' applications, as well as being able to accommodate and solve custom applications. Their extremely large range of customization is driven by the variety they offer in the caster, wheel, and wheel bearing arrangements. Because of this large range of customization, stockpiling combinations of caster wheels is not an option. Therefore, quick turnaround from customer order to shipping is needed. This process is further complicated by Albion's demand for small, custom orders from its customers.

One of RM's most attractive features is its ability to produce parts without the use of molds or tooling. In traditional manufacturing enterprises, this results in an elimination of the large, upfront tooling costs that usually accompany traditional manufacturing technologies. The 'zero-tooling' ability results in manufacturers having the ability to produce small lot size, custom geometry at relatively low cost. When using traditional manufacturing technologies, small custom orders are usually accompanied by a significantly higher cost. In this example, we explore the use of RM to manufacture custom caster wheels as a way to reduce the cost and manufacture time of caster wheels that are manufactured using traditional manufacturing technologies.

\subsubsection{Albion and Rapid Manufacturing}

The following attributes are considered key factors to the success of Albion in the manufacturing environment in which they operate:

\section{Variety}

Albion operates in an environment where variety is key. Albion has created a large variety of products to accommodate most of their customer needs. Custom products are offered for applications that are not covered by their product line.

\section{Design and Production time}

Design and production time are primary drivers of the current consumer marketplace. Given the manufacturing environment in which Albion operates, short design and 
production times are desirable. Customers require a fast turnaround of product. Providing this turnaround affords Albion a sustainable competitive advantage.

\section{Material Variety}

With the large variety of products that Albion offers comes a large variety of materials offered. The manufacturing process for Albion must be able to handle this large range of materials and the complexity that comes along with it.

\section{Manufacturing}

Given Albion's existing operating practices, the manufacturing process must be able to handle direct and indirect fabrication techniques. Utilizing an operation that can handle both of these fabrication techniques will increase the flexibility of Albion's operation and give options within the production method.

Because of the manufacturing environment in which they operate, Albion provides an excellent opportunity for the integration of RM technologies into manufacturing systems. This is largely due to their large variety and customization options offered and the quick turnaround expected by their customers. RM will be especially useful in dealing with the custom orders that Albion receives. When compared to traditional manufacturing technologies, RM offers the following advantages:

- RM offers the ability to produce multiple custom caster wheel configurations in one build. This ability allows Albion the flexibility to produce small to large lot orders, as well as combination orders, without much forward planning. RM does not require any special tooling or molds. Caster wheel molds cost an average of $\$ 3000$ and 3 months (when outsourced to China) to manufacture. This significant upfront tooling cost and manufacture time greatly affects the price and manufacturing time of small to medium lot size orders of caster wheels that Albion receives. Remember, at small lot sizes, this cost cannot be marginalized by the production of a large number of wheels. 
- RM also allows the manufacturer, and the customer, the ability to produce truly custom caster wheels. RM offers geometric complexity at no extra cost, whereas with traditional manufacturing process, the cost to manufacture the part increases as the complexity increases.

Because of the above advantages, RM is a good candidate for the manufacturing of custom caster wheels.

\subsubsection{Project Scope}

As stated earlier, Albion produces a wide range of products. These products, specifically the caster wheels, are made of various materials suited for different applications. These wheel materials include cast iron, steel, nylon, polyurethane, phenolic, rubber, and combinations of the listed materials. In the situation presented in this example, the author will only consider the direct manufacture of custom, steel caster wheels. Given this scope, the author will only consider metal RM technologies.

Due to the scope of the project, only the class of metal RM technologies will be considered. These technologies will enable the direct production of steel caster wheels. The technologies are classified in the Figure 5.2. 


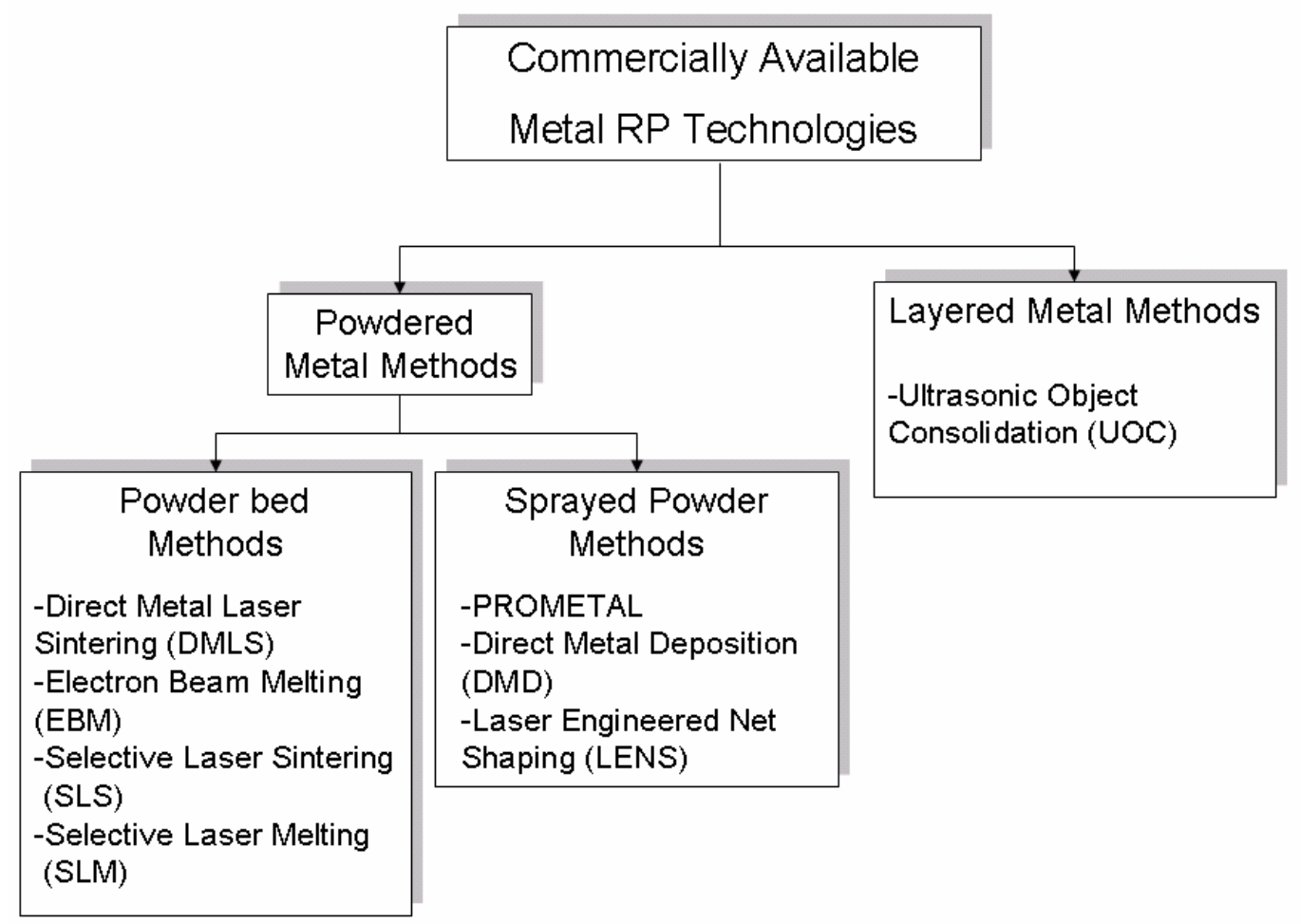

Figure 5. 2 Commercially Available Metal RM Technologies

As seen in the Figure 5.2, the technologies are classified into 2 major methods: those that use powdered metal and those that use layered metal. Powdered metal technologies can be broken down into those that use a powder bed (with a laser tracing each cross-section) and those that spray powder (to create each cross-section).

The technologies are described in the Table 5.1.

Table 5. 1 Table of Commercially-Available RM Technologies for Metal

\begin{tabular}{|c|c|c|c|c|}
\hline RM Technology & $\begin{array}{l}\text { Class } \\
\text { (Approach) }\end{array}$ & $\begin{array}{l}\text { Working Principle } \\
\text { (Fabrication Technique) }\end{array}$ & $\begin{array}{l}\text { Materials } \\
\text { (metal) }\end{array}$ & $\begin{array}{l}\text { Advantages (+) } \\
\text { / Disadvantages (- }\end{array}$ \\
\hline $\begin{array}{l}\text { PROMETAL } \\
\text { Extrude-Hone }\end{array}$ & $\begin{array}{l}\text { Laser Printing } \\
\text { (Metallic } \\
\text { Powder }+ \\
\text { Binder) }\end{array}$ & $\begin{array}{l}\text { Similar to } 3 \text { D printing, a printer head is } \\
\text { used to selectively deposit a binder } \\
\text { polymer over a sheet of powdered } \\
\text { metal. The low density "green" part is } \\
\text { then infiltrated with another metal to } \\
\text { make it dense. } \\
\text { (Direct + PostP + Fin) }\end{array}$ & $\begin{array}{l}\text {-Prometal S3 } \\
\text { (SS316 + } \\
\text { bronze) } \\
\text {-Prometal S4 } \\
\text { (SS420 + } \\
\text { bronze) } \\
\text {-Prometal S4H } \\
\text { (SS420 + } \\
\text { bronze) }\end{array}$ & $\begin{array}{l}(+) \text { fast } \\
(+) \text { low cost } \\
(-) \text { sometime } \\
\text { produces fragile } \\
\text { parts }\end{array}$ \\
\hline
\end{tabular}


Table 5. 1 Continued

\begin{tabular}{|c|c|c|c|c|}
\hline $\begin{array}{l}\text { Ultrasonic Object } \\
\text { Consolidation } \\
\text { (UOC) } \\
\text { Solidica, Inc. }\end{array}$ & $\begin{array}{l}\text { Layered Metal } \\
\text { (Direct + Fin) }\end{array}$ & $\begin{array}{l}\text { UOC involves a high intensity } \\
\text { ultrasonic energy source to join layers } \\
\text { of metal foil together. The layer is then } \\
\text { contour milled to create the layer } \\
\text { profile. } \\
\text { (Direct + Fin) }\end{array}$ & Aluminum & $\begin{array}{l}\text { (+) creates } 98-99 \% \\
\text { dense parts } \\
(+) \text { laser-less } \\
\text { process } \\
(+) \text { no post- } \\
\text { processing needed } \\
(-) \text { limited complex } \\
\text { geometry }\end{array}$ \\
\hline $\begin{array}{l}\text { Laser Engineering } \\
\text { Net Shaping } \\
\text { (LENS) } \\
\\
\text { Optomec Design } \\
\text { Company }\end{array}$ & $\begin{array}{l}\text { Sprayed } \\
\text { Powder } \\
\text { (Metallic } \\
\text { Powder) }\end{array}$ & $\begin{array}{l}\text { LENS uses a high powered Nd:YAG } \\
\text { laser to melt powdered metals one layer } \\
\text { at a time. The metal powder is fed } \\
\text { through a nozzle onto the workpiece } \\
\text { while being fused by the laser. } \\
\text { (Direct + Fin) }\end{array}$ & $\begin{array}{l}\text {-aluminum } \\
\text {-copper } \\
\text {-Inconel } \\
\text {-stainless steel } \\
\text {-tool steel } \\
\text {-titanium }\end{array}$ & $\begin{array}{l}\text { (+) wide range of } \\
\text { materials } \\
(+) \text { good build } \\
\text { plane accuracy } \\
(+) \text { produces } 100 \% \\
\text { dense parts } \\
(+) \text { can build multi- } \\
\text { material parts } \\
(+) \text { no post } \\
\text { processing needed } \\
(-) \text { poor accuracy in } \\
\text { growth direction } \\
(-) \text { poor surface } \\
\text { finish } \\
(-) \text { limited complex } \\
\text { geometry }\end{array}$ \\
\hline $\begin{array}{l}\text { Direct Metal } \\
\text { Deposition (DMD) } \\
\text { The POM Group, } \\
\text { Inc / Trumpf (w/o } \\
\text { feedback system) }\end{array}$ & $\begin{array}{l}\text { Sprayed } \\
\text { Powder } \\
\text { (Metallic } \\
\text { Powder) }\end{array}$ & $\begin{array}{l}\text { Pure metal powder is sprayed coaxially } \\
\text { to a } \mathrm{CO}_{2} \text { laser beam onto a molten pool. } \\
\text { This, coupled with a patented (POM) } \\
\text { closed-loop feedback system to assure } \\
\text { quality, produces a fully dense material } \\
\text { part. } \\
\text { (Direct + Fin) }\end{array}$ & $\begin{array}{l}\text {-tool steel alloys } \\
\text { (Fe,C,Cr,V):P20, } \\
\text { P21,H13,S7,D2 } \\
\text {-Stainless } \\
\text { Steel:420SS,319 } \\
\text { SS,174SS } \\
\text {-Cobalt-based } \\
\text { Alloys } \\
\text { (Co,Cr,Mo,Ni) } \\
\text {-Super alloys } \\
\text { (Co,Mo,Cr,Si) } \\
\text {-Copper-based } \\
\text { Alloys }(\mathrm{CuNi})^{8}\end{array}$ & $\begin{array}{l}(+) \text { material } \\
\text { properties equal or } \\
\text { exceed those of } \\
\text { conventional } \\
\text { quenched and } \\
\text { tempered wrought } \\
\text { irons. } \\
(+) \text { produces fully } \\
\text { dense parts } \\
(+) \text { multi-material } \\
\text { parts } \\
(-) \text { limited complex } \\
\text { geometry } \\
(-) \text { quite slow }\end{array}$ \\
\hline $\begin{array}{l}\text { Selective Laser } \\
\text { Sintering }\end{array}$ & $\begin{array}{l}\text { Layered } \\
\text { Powder } \\
\text { (Metallic } \\
\text { Powder }+ \\
\text { Binder) }\end{array}$ & $\begin{array}{l}\text { SLS uses a } \mathrm{CO}_{2} \text { laser to fuse and sinter } \\
\text { metallic powder layer by layer. The } \\
\text { laser selectively sinters the cross } \\
\text { section of the part. For indirect } \\
\text { fabrication, a polymer coated metal } \\
\text { powder is used to make the "green" } \\
\text { part. The part is then infiltrated with } \\
\text { another metal (usually copper) and } \\
\text { sintered. } \\
\text { (Direct + PostP + Fin) }\end{array}$ & $\begin{array}{l}\text {-Laserform ST- } \\
100 \text { (steel }+ \\
\text { bronze) } \\
\text {-Laserform ST- } \\
200 \text { (steel + } \\
\text { bronze) } \\
\text {-Laserform A6 } \\
\text { steel }\end{array}$ & $\begin{array}{l}\text { (+) accurate in } \\
\text { build and growth } \\
\text { direction } \\
(+) \text { capable of } \\
\text { producing complex } \\
\text { shapes } \\
\text { (-)decreased } \\
\text { accuracy because } \\
\text { of shrinkage } \\
(-) \text { small range of } \\
\text { materials } \\
(-) \text { residual stresses }\end{array}$ \\
\hline
\end{tabular}


Table 5. 1 Continued

\begin{tabular}{|c|c|c|c|c|}
\hline $\begin{array}{l}\text { Direct Metal Laser } \\
\text { Sintering (DMLS) } \\
\text { EOS }\end{array}$ & $\begin{array}{l}\text { Layered } \\
\text { Powder } \\
\text { (Metallic } \\
\text { Powder) }\end{array}$ & $\begin{array}{l}\text { Uses } 200 \mathrm{~W} \mathrm{CO}_{2} \text { laser (EOSINT M } \\
\text { 250) or a } 200 \mathrm{~W} \text { ytterbium fibre laser } \\
\text { (EOSINT M 270) to sinter metal } \\
\text { powder without binder or fluxing agent. } \\
\text { (Direct + PostP+ Fin) }\end{array}$ & $\begin{array}{l}\text {-DirectMetal20 } \\
\text { (bronze-based) } \\
\text {-DirectMetal50 } \\
\text {-DirectSteel }\end{array}$ & $\begin{array}{l}(+) \text { good surface } \\
\text { quality } \\
(+) \text { mechanical } \\
\text { properties of } \\
\text { existing metal } \\
(+) \text { no post } \\
\text { processing needed } \\
(+) \text { can be } \\
\text { infiltrated to full } \\
\text { density }\end{array}$ \\
\hline $\begin{array}{l}\text { Direct Metal Laser } \\
\text { Melting (DMLM) } \\
\text { Trumpf }\end{array}$ & $\begin{array}{l}\text { Layered } \\
\text { Powder } \\
\text { (Metallic } \\
\text { Powder) }\end{array}$ & $\begin{array}{l}\text { Same as DMLS, but uses solid state } \\
\text { laser. Melts the metal, as opposed to } \\
\text { sintering it as in DMLS. } \\
\text { (Direct + Fin) }\end{array}$ & $\begin{array}{l}\text {-tool steel } \\
\text {-stainless steel } \\
\text {-titanium }\end{array}$ & $\begin{array}{l}(+) \text { good surface } \\
\text { quality } \\
(+) \text { mechanical } \\
\text { properties of } \\
\text { existing metal } \\
(+) \text { no post } \\
\text { processing needed }\end{array}$ \\
\hline $\begin{array}{l}\text { Electron Beam } \\
\text { Melting (EBM) } \\
\text { Arcam AB }\end{array}$ & $\begin{array}{l}\text { Layered } \\
\text { Powder } \\
\text { (Metallic } \\
\text { Powder) }\end{array}$ & $\begin{array}{l}\text { Uses a } 4 \mathrm{~kW} \text { electron beam to melt } \\
\text { metal powder layer by layer. After a } \\
\text { preheating step for stability and } \\
\text { reduction of thermal gradients, each } \\
\text { layer is melted by increasing beam } \\
\text { power and decreasing speed. The } \\
\text { process takes place in a vacuum, so that } \\
\text { the electrons have a clear path to the } \\
\text { metal. } \\
\text { (Direct + Fin) }\end{array}$ & $\begin{array}{l}-\mathrm{H} 13 \text { tool steel } \\
\text {-low alloy steel } \\
200\end{array}$ & $\begin{array}{l}(+) \text { EB technology } \\
\text { more efficient than } \\
\text { laser } \\
(+) \text { high strength } \\
\text { properties because } \\
\text { of vacuum } \\
(+) \text { impurity free } \\
(+) \text { residual } \\
\text { stresses minimized } \\
(+) \text { fast (high build } \\
\text { speeds) } \\
(+) \text { no post } \\
\text { processing needed } \\
(-) \text { increased cost } \\
\text { because of vacuum }\end{array}$ \\
\hline $\begin{array}{l}\text { Selective Laser } \\
\text { Melting (SLM) } \\
\text { Fockele and } \\
\text { Schwarze }\end{array}$ & $\begin{array}{l}\text { Layered } \\
\text { Powder } \\
\text { (Metallic } \\
\text { Powder) }\end{array}$ & $\begin{array}{l}\text { With SLM, market-common one- } \\
\text { component pure metal powders (w/o } \\
\text { binders) is locally melted by an infrared } \\
\text { laser beam layer by layer. } \\
\text { (Direct + Fin) }\end{array}$ & $\begin{array}{l}\text {-stainless steel } \\
\text {-tool steel } \\
\text { Corn size: } 10-30 \\
\text { microns }\end{array}$ & $\begin{array}{l}(+) \text { no specialty } \\
\text { materials needed } \\
(+)>99 \% \text { dense } \\
\text { parts } \\
(+) \text { no post } \\
\text { processing needed }\end{array}$ \\
\hline
\end{tabular}

In the table above, post processing (PostP) is defined as processes needed to complete the densification of the part (infiltration, etc.). Finishing (Fin) is defined as processes needed to complete the shape of the surface of the part (machining, polishing, etc.). Also, indirect fabrication is the use of RM technologies to generate molds or mold inserts for parts. Direct fabrication is defined as the use of RM technology for the direct production of a part. 
Given the technologies in Table 5.1, Table 5.2 was generated to highlight specific attributes related to Albion Inc.

Table 5. 2 Highlighted Technology Attributes

\begin{tabular}{|c|c|c|c|c|c|c|}
\hline & Density & Complex & Material & & Post & Finishing \\
\hline & & Geometry & Steel & Alum & Processing & \\
\hline PROMETAL & $\begin{array}{l}60 \% \text { metal, } \\
40 \% \\
\text { Organic } \\
\text { Binder, } \\
\text { then } \\
\text { infiltrated } \\
\text { with bronze }\end{array}$ & Yes & $\begin{array}{l}\text { Stainless } \\
\text { Steel }\end{array}$ & no & $\begin{array}{l}\text { Yes, needs to be } \\
\text { infiltrated with } \\
\text { bronze }\end{array}$ & Yes \\
\hline UOC & $\begin{array}{l}98-99 \% \\
\text { dense }\end{array}$ & $\begin{array}{l}\text { Limited, due } \\
\text { to limited } \\
\text { ability to } \\
\text { build } \\
\text { overhangs. }\end{array}$ & No & Yes & No & $\begin{array}{l}\text { No, contour- } \\
\text { milled after } \\
\text { every layer }\end{array}$ \\
\hline DMD & $\begin{array}{l}100 \% \\
\text { dense }\end{array}$ & $\begin{array}{l}\text { Limited, due } \\
\text { to limited } \\
\text { ability to } \\
\text { build } \\
\text { overhangs }\end{array}$ & Yes & No & No & Yes \\
\hline SLS & $\begin{array}{l}60 \% \text { steel, } \\
40 \% \\
\text { binder, then } \\
\text { infiltrated } \\
\text { with bronze }\end{array}$ & Yes & Yes & No & Yes & Yes \\
\hline LENS & $\begin{array}{l}100 \% \\
\text { dense }\end{array}$ & $\begin{array}{l}\text { Limited, due } \\
\text { to limited } \\
\text { ability to } \\
\text { build } \\
\text { overhangs } \\
\text { (optional 5- } \\
\text { axis head) }\end{array}$ & Yes & Yes & No & Yes \\
\hline DMLS & $\begin{array}{l}>80 \% \\
\text { dense, } \\
100 \% \text { if } \\
\text { infiltrated }\end{array}$ & Yes & Yes & No & $\begin{array}{l}\text { Maybe, for } \\
\text { densification }\end{array}$ & Yes \\
\hline DMLM & $\begin{array}{l}100 \% \\
\text { dense }\end{array}$ & Yes & Yes & No & No & Yes \\
\hline EBM & $\begin{array}{l}100 \% \\
\text { dense }\end{array}$ & Yes & Yes & No & No & Yes \\
\hline SLM & $\begin{array}{l}>99 \% \\
\text { dense }\end{array}$ & Yes & Yes & No & No & Yes \\
\hline
\end{tabular}

Based on the project scope, PROMETAL and UOC were eliminated from further consideration because they do not provide steel as a material choice. DMLM was also eliminated due to the infancy of the technology. 


\subsubsection{RM Technology Requirements}

The specific requirements for the RM technology are as follows:

\section{Production}

In Albion's operating environment, production time is a very critical constraint. Therefore, minimizing the time it takes to make the caster wheels will reduce the production time of the parts. In particular, with the caster wheels considered in this example (which will be used for custom applications), this time will be a significant factor.

\section{Easy operation}

The technology must be easily operable. When dealing with custom, short-run products, it is sometimes efficient to have the caster wheels manufactured in-house by engineers, as opposed to outsourcing them with increased cost. With that, there is a need for the technology to accommodate a wide range of users within Albion's ranks.

\section{Minimal cost}

Holding quality constant across a range of products, reduced cost makes products more attractive to the customer. Reducing the manufacturing cost, the manufacturing technology in this case, of a product reduces the cost to the customer. In this case, the elimination of the mold tooling cost will equate to a cheaper price for the customer. In cases where time is critical, cost is not as important.

\section{Surface Finish}

For surface finish, the caster wheel must be divided into 2 parts: the core and the tire. In some constructions, this wheel core and tire are a single construction. Surface finish of the caster core is not very important, thus a medium surface finish is desired. A low surface finish may give the appearance of a low quality product.

Part size 
The manufacturing process must be able to accommodate the maximum size of product that can be produced. For the direct fabrication technique, the operation must be able to accommodate the maximum size of the caster wheel.

Based on the above requirements, a selection can now be performed. The selection was performed using Selection for RM method (Section 5.1.4) and Selection DSP (5.1.5).

\subsubsection{Selection for Rapid Manufacturing}

Before beginning the selection process, the uncertainty involved in the customization process was considered. Since these caster wheels will be customized, there is a degree of geometric uncertainty involved.

\section{Step 1. Characterize the uncertainty involved}

In this step, the range of customization is qualitatively assessed. In this example, we have decided to only allow customization of certain features. This example will only deal with the customization of all-steel caster wheels. It should also be noted that only standard 1.25 in. diameter $\mathrm{x} 4$ in. length bolts will be used for the inner bore, therefore these dimensions will be constrained. The customers will be allowed to customize all other features of the caster wheel.

After the range of customization is defined qualitatively, a quantitative assessment must be performed. The designer should define which geometric dimensions will be constrained (certain) and which will be bounded (uncertain). The profile of the caster wheel is displayed in Figure 5.3. 


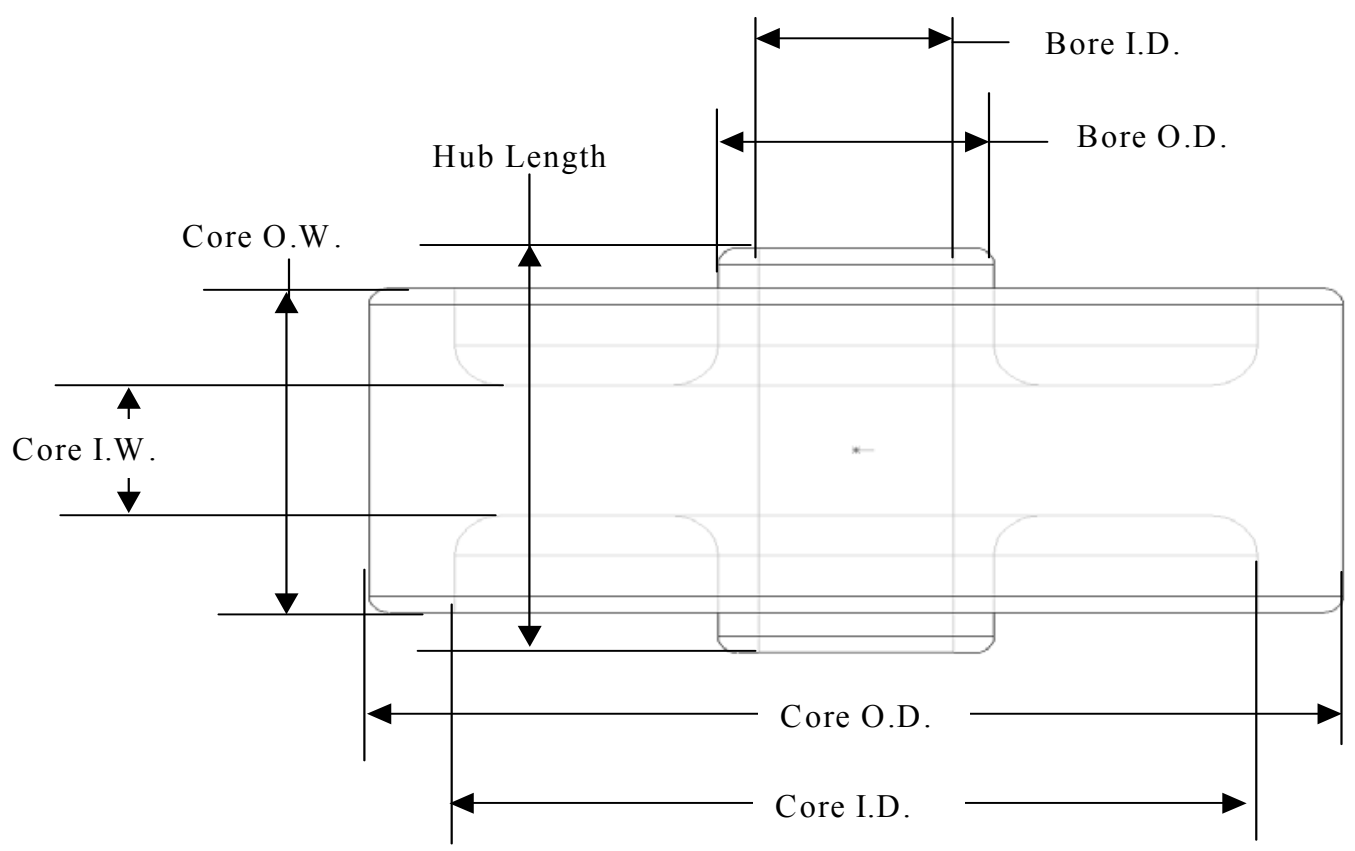

Figure 5. 3 Caster wheel side profile

The uncertainty is quantified using constraints and bounds on the above dimensions. The constraints and bounds used for this example are displayed in Table 5.3.

Table 5. 3 Caster wheel dimensions

\begin{tabular}{|c|c|c|}
\hline & \multicolumn{2}{|c|}{ Dimensions } \\
\hline & $\min$ & $\max$ \\
\hline Core Outer Diameter & 4 & 6 \\
\hline Core Inner Diameter & 3.5 & 5.5 \\
\hline Bore Outer Diameter & 1.5 & 2.25 \\
\hline Bore Inner Diameter & 1.25 & 1.25 \\
\hline Hub Length & 2.5 & 2.5 \\
\hline Core Outer Width & 1.5 & 3 \\
\hline Core Inner Width & 0.5 & 1.25 \\
\hline
\end{tabular}

As displayed in the table, the uncertain dimensions are displayed as interval sets. The constrained dimensions are constrained by the standard size of the bolt used in the assembly process.

Step 2. Describe the alternatives and provide acronyms 
The alternatives are as follows: Direct Metal Laser Sintering (DMLS), Direct Metal Deposition (DMD), Electron Beam Melting (EBM), Laser Engineered Net Shaping (LENS), Selective Laser Sintering (SLS), and Selective Laser Melting (SLM). Descriptions are provided in Table 5.1.

\section{Step 3. Describe each relevant attribute, specify its relative importance and provide acronyms}

The attributes are described as follows:

Ultimate Tensile Strength (UTS): UTS is the maximum stress reached before a material fractures.

Rockwell Hardness C (Hard): Hardness is the commonly defined as the resistance of a material to indentation.

Density (Dens.): The density refers to the final density of the part after all processing steps. This density is proportional to the amount of voids found at the surface. These voids cause a rough surface finish.

Detail Capability (DC): The detail capability is the smallest feature size the technology can make.

Geometric Complexity (GC): The geometric complexity is the ability of the technology to build complex parts. More specifically, in this case, it is used to refer to the ability to produce overhangs, since this is the most critical limitation with respect to producing complex parts.

Build Time (Time): The build time refers to the build time of a part, not including post processing steps.

Part Cost (Cost): The part cost is the cost it takes to build one part with all costs included. These costs include manufacturing cost, material cost, machine cost, operation cost, etc.

In this example, we examine 2 weighting scenarios (relative importance ratings). In Scenario 1, a pairwise comparison was used to determine relative importance of each attribute. In this scenario, geometric complexity was most heavily weighted because of 
the significant overhangs present in the build orientation of the casters. Build time and part cost are also heavily weighted because of their importance to the business structure surrounding customization of caster wheels. Because of the environment of use of the caster wheels, UTS was also given a high weighting. Detail capability was weighted least because of the lack of small, detailed features in the geometry of the caster wheels. In Scenario 2, all selection attributes were equally weighted. The relative importance weightings for each scenario are presented in Table 5.4.

\section{Step 4: Specify scales, acceptable range of values, and rate the alternatives with respect to each attribute.}

At this step, bounded geometric characteristics (such as part volume, area, etc.) are calculated using interval arithmetic operations on the bounded and constrained geometric dimensions. In our case, the particular geometric constraint of concern is the bounded part volume, which is used to calculate the build time and part cost. Based on the uncertainty in the geometric dimensions in Table 5.3, the volume of the caster wheel is calculated (using interval arithmetic) using Eq. 5.1.

$$
\begin{gathered}
\text { vol }=c o w \cdot \frac{\pi}{4} \cdot \operatorname{cod}^{2}+(\operatorname{cow}-c i w) \cdot \frac{\pi}{4} \cdot\left(\operatorname{cod}^{2}-c i d^{2}+b o d^{2}-b i d^{2}\right) \\
+(h l-c o w) \cdot \frac{\pi}{4} \cdot\left(b o d^{2}-b i d^{2}\right)
\end{gathered}
$$

$$
\begin{aligned}
& \text { vol }=[1.5,3] \cdot \frac{\pi}{4} \cdot[4,6]^{2}+([1.5,3]-[0.5,1.25]) \cdot \frac{\pi}{4} \cdot\left([4,6]^{2}-[3.5,5.5]^{2}\right. \\
&\left.+[1.5,2.25]^{2}-1.25^{2}\right)+(2.5-[1.5,3]) \cdot \frac{\pi}{4} \cdot\left([1.5,2.25]^{2}-1.25^{2}\right) \\
&=\left[-3.6 \cdot 10^{5}, 1.5 \cdot 10^{6}\right]
\end{aligned}
$$

In Eq. 5.1, $c o d=$ core outer diameter, $\operatorname{cid}=$ core inner diameter, $c o w=$ core outer width, $c i w=$ core inner width, $b o d=$ bore outer diameter, $b i d=$ bore inner diameter, and $h l=$ hub length from Fig. 5.3. When using interval arithmetic, if variables are repeated, as in the case of Eq. 5.1, the calculations will yield a very conservative result. However, our 
answer will still be bounded by this result. Because of the conservativeness of interval arithmetic approach in our example, the uncertainty was further reduced using a brute force approach. In the brute force approach, a less conservative bound of the uncertainty was found by using arithmetic operations on the minimum and maximum dimensional bounds in a logical manner. In other words, using the geometric equations for the volume of the caster wheel, the maximum and minimum volumes were calculated by setting the dimensional parameters to either maximum or minimum bound. For example, to calculate the maximum volume, cow, cod, hl, ciw, bid and bod were maximized, while cid was minimized. The uncertainty in the part volume was reduced to $\left[1.7^{*} 10^{5}, 1.2^{*} 10^{6}\right]$ $\mathrm{mm}^{3}$.

This uncertainty is then propagated to the selection attributes. For example, using Eq. 5.2, the build time for DMD is calculated as follows:

$$
\begin{gathered}
\text { Build_Time_avg }=\frac{\text { part_volume }}{\text { build_rate }} \\
D M D_{\text {build_time }}=\frac{\left[1.7 \cdot 10^{5}, 1.2 \cdot 10^{6}\right] \mathrm{mm}^{3}}{4.5 \mathrm{~mm}^{3} / \mathrm{sec}}=[10.43,72.25] \mathrm{hrs}
\end{gathered}
$$

Part cost was calculated using the cost models presented in Chapter 4.

The alternative ratings as well as the acceptable, range of values for each attribute, are presented in Table 5.4. 
Table 5. 4 Attribute Ratings

\begin{tabular}{|c|c|c|c|c|c|c|c|c|c|c|c|}
\hline & \multicolumn{10}{|c|}{ Attributes } \\
\hline & & \multirow[t]{2}{*}{ UTS } & \multirow[t]{2}{*}{ Hard } & \multirow[t]{2}{*}{ Density } & \multirow[t]{2}{*}{$\begin{array}{l}\text { Detail } \\
\text { Cap. }\end{array}$} & \multicolumn{2}{|c|}{ Geom. Compl. } & \multicolumn{2}{|c|}{ Build Time avg } & \multicolumn{2}{|c|}{ Part Cost } \\
\hline & & & & & & MIIn & $\operatorname{Tax}$ & MIIn & $\operatorname{Thax}$ & MIn & $\operatorname{Thax}$ \\
\hline \multicolumn{2}{|c|}{ Rel. Imp } & 0.167 & 0.143 & 0.071 & 0.024 & 0.214 & 0.214 & 0.190 & 0.190 & 0.190 & 0.190 \\
\hline \multirow{6}{*}{ 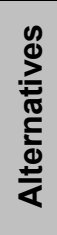 } & DMD & 1800 & 53 & 100 & 1.016 & 4 & 6 & 10.43 & 72.25 & 29.48 & 168.15 \\
\hline & DMLS & 600 & 21 & 95 & 0.3 & 7 & 10 & 17.00 & 117.79 & 386.98 & 2045.93 \\
\hline & EBM & 1430 & 50 & 100 & 1.2 & 7 & 10 & 4.27 & 29.56 & $\overline{134.41}$ & 508.56 \\
\hline & LENS & 1703 & 53 & 100 & 0.762 & 4 & 6 & 2.06 & 14.28 & 64.17 & 306.52 \\
\hline & SLM & 2000 & 60 & 99.5 & 0.15 & 7 & 10 & 11.25 & 77.96 & 237.43 & 1340.57 \\
\hline & SLS & 606 & 15 & 100 & 0.6 & 7 & 10 & 17.00 & $\begin{array}{l}117.79 \\
\end{array}$ & 180.67 & 889.63 \\
\hline \multirow{5}{*}{ 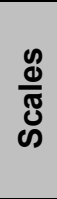 } & Type & Ratio & Ratio & Ratio & Ratio & \multicolumn{2}{|c|}{ Interval } & \multicolumn{2}{|c|}{ Ratio } & \multicolumn{2}{|c|}{ Ratio } \\
\hline & low & 500 & 10 & 95 & 2 & \multicolumn{2}{|c|}{1} & \multicolumn{2}{|c|}{2} & \multirow{2}{*}{\multicolumn{2}{|c|}{$\frac{25}{1000}$}} \\
\hline & high & 2500 & 70 & 100 & 0.1 & \multicolumn{2}{|c|}{10} & \multicolumn{2}{|c|}{120} & & \\
\hline & pref & 2500 & 70 & 100 & 0.1 & \multicolumn{2}{|c|}{10} & \multicolumn{2}{|c|}{0.1} & \multicolumn{2}{|c|}{25} \\
\hline & Units & $\mathrm{Mpa}$ & HRc & \begin{tabular}{|l|} 
percent \\
\end{tabular} & $\mathrm{mm}$ & \multicolumn{2}{|c|}{$\mathrm{nmu}$} & \multicolumn{2}{|c|}{ hrs } & \multicolumn{2}{|c|}{ USD } \\
\hline
\end{tabular}

\section{Step 5: Normalize the attribute ratings}

The attribute ratings in Table 5.4 were normalized using the equations presented in

Section 3.2.3. The normalized attribute ratings are presented in Table 5.5.

Table 5. 5 Normalized Attribute Ratings

\begin{tabular}{|c|c|c|c|c|c|c|c|c|c|c|c|}
\hline & \multicolumn{10}{|c|}{ Attributes } \\
\hline & & \multirow[t]{2}{*}{ UTS } & \multirow[t]{2}{*}{ Hard } & \multirow[t]{2}{*}{ Density } & \multirow[t]{2}{*}{$\begin{array}{l}\text { Detail } \\
\text { Cap. }\end{array}$} & \multicolumn{2}{|c|}{ Geom. Compl. } & \multicolumn{2}{|c|}{ Build Time_avg } & \multicolumn{2}{|c|}{ Part Cost } \\
\hline & & & & & & Min & $\operatorname{Max}$ & Min & $\operatorname{Max}$ & Min & Max \\
\hline & Scen 1 & 0.167 & 0.143 & 0.071 & 0.024 & 0.214 & 0.214 & 0.190 & 0.190 & 0.190 & 0.190 \\
\hline & Scen 2 & 0.143 & 0.143 & 0.143 & 0.143 & 0.143 & 0.143 & 0.143 & 0.143 & 0.143 & 0.143 \\
\hline \multirow{6}{*}{ 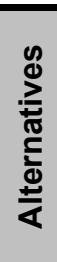 } & $\overline{D M D}$ & 0.650 & 0.717 & 1.000 & 0.518 & 0.333 & 0.556 & 0.929 & 0.405 & 0.995 & 0.853 \\
\hline & DMLS & 0.050 & 0.183 & 0.000 & 0.895 & 0.667 & 1.000 & 0.873 & 0.019 & 0.629 & 0.000 \\
\hline & EBM & 0.465 & 0.667 & 1.000 & 0.421 & 0.667 & 1.000 & 0.981 & 0.766 & 0.888 & 0.504 \\
\hline & LENS & 0.602 & 0.717 & 1.000 & 0.652 & 0.333 & 0.556 & 0.999 & 0.896 & 0.960 & 0.711 \\
\hline & SLM & 0.750 & 0.833 & 0.900 & 0.974 & 0.667 & 1.000 & 0.922 & 0.356 & 0.782 & 0.000 \\
\hline & SLS & 0.053 & 0.083 & 1.000 & 0.737 & 0.667 & 1.000 & 0.873 & 0.019 & 0.840 & 0.113 \\
\hline
\end{tabular}

\section{Step 6: Evaluate the merit functions}

The merit function values of the alternatives (Scenario 1 and 2) are displayed in Table 5.6. As explained earlier, the merit function intervals are a function of the uncertainty range. For example, the merit function intervals are calculated (using interval arithmetic) for DMD as follows: 


$$
\begin{aligned}
M F_{D M D} & =\sum_{J=1} I_{i} \cdot N R_{i j}=0.17 \cdot 0.65+0.14 \cdot 0.72+0.071 \cdot 1+0.024 \cdot 0.52 \\
& +0.21 \cdot[0.33,0.56]+0.19 \cdot[0.93,0.41]+0.19 \cdot[0.99,0.85]=[0.73,0.65]
\end{aligned}
$$

Table 5. 6 Alternative Merit Function Values for Scenario 1 and 2

\begin{tabular}{|c|c|c|c|c|}
\cline { 2 - 5 } \multicolumn{1}{c|}{} & \multicolumn{3}{c|}{ Merit Function } \\
\cline { 2 - 5 } \multicolumn{1}{c|}{} & \multicolumn{2}{c|}{ Scenario 1 } & \multicolumn{2}{c|}{ Scenario 2 } \\
\cline { 2 - 5 } \multicolumn{1}{c|}{$\min$} & max & min & max \\
\hline DMD & 0.73 & 0.65 & 0.73 & 0.67 \\
\hline DMLS & 0.48 & 0.27 & 0.47 & 0.31 \\
\hline EBM & 0.75 & 0.71 & 0.73 & 0.69 \\
\hline LENS & 0.73 & 0.71 & 0.75 & 0.73 \\
\hline SLM & 0.80 & 0.61 & 0.83 & 0.69 \\
\hline SLS & 0.58 & 0.35 & 0.61 & 0.43 \\
\hline
\end{tabular}

Based on the overlap of the merit function intervals, dominance of one alternative over another cannot be definitively established. Therefore, selection criteria must be used to rank the alternatives. For the Hurwicz criterion, a decision maker's decision preference, $\alpha$, of 0.3 was determined after performing the lottery in Fig. 2.8. The selection parameters for the selection criteria are displayed in Tables 5.7 and 5.8.

Table 5. 7 Selection parameters for Decision Theory selection criteria (Scenario 1)

\begin{tabular}{|c|c|c|c|c|}
\cline { 2 - 5 } \multicolumn{1}{c|}{} & Maximin & Maximax & Hurwicz & Laplace \\
\hline DMD & 0.65 & 0.73 & 0.68 & 0.69 \\
\hline DMLS & 0.27 & 0.48 & 0.34 & 0.38 \\
\hline EBM & 0.71 & 0.75 & 0.72 & 0.73 \\
\hline LENS & 0.71 & 0.73 & 0.72 & 0.72 \\
\hline SLM & 0.61 & 0.80 & 0.67 & 0.71 \\
\hline SLS & 0.35 & 0.58 & 0.42 & 0.46 \\
\hline
\end{tabular}


Table 5. 8 Selection parameters for Decision Theory selection criteria (Scenario 2)

\begin{tabular}{|c|c|c|c|c|}
\cline { 2 - 5 } \multicolumn{1}{c|}{} & Maximin & Maximax & Hurwicz & Laplace \\
\hline DMD & 0.67 & 0.73 & 0.69 & 0.70 \\
\hline DMLS & 0.31 & 0.47 & 0.36 & 0.39 \\
\hline EBM & 0.69 & 0.73 & 0.70 & 0.71 \\
\hline LENS & 0.73 & 0.75 & 0.74 & 0.74 \\
\hline SLM & 0.69 & 0.83 & 0.73 & 0.76 \\
\hline SLS & 0.43 & 0.61 & 0.48 & 0.52 \\
\hline
\end{tabular}

Since the Maximin and Maximax criteria can be seen as extreme cases of decision maker's decision preference in the Hurwicz criterion, we will not consider them further. In essence, when comparing the Hurwicz and the Laplace criteria, the decision maker is deciding whether to evaluate the alternatives based on average performance in the case of Laplace criterion, or based on decision maker's decision preference in the case of the Hurwicz criterion. We believe that both of these decision criteria should be considered in the selection process and a criterion selected based on the type of decision problem. The limitations and advantages of the selection criteria are discussed further in the Section 5.3 .

\section{Step 7: Post Solution Analysis and Verification of results}

As seen in Table 5.7, for Scenario 1, EBM and LENS ranked atop the other alternatives for both the Hurwicz and Laplace criteria. This is largely due to the high build time and part cost ratings for these alternatives. In Scenario 2, equal importance was given to all attributes. In this scenario, we can see how the use of different selection criteria can lead to conflicting results as shown in Table 5.8. In the case of the Hurwicz criterion, SLM and LENS ranked atop the other alternatives. In the case of the Laplace criterion, SLM is the top performer, followed by LENS and EBM. Although SLM and LENS distinguished themselves as top performers in both cases, a single top performer cannot be established based on the conflicting rankings for this scenario.

As part of the sensitivity analysis, the effect of decision maker's decision preferences on the results of the Hurwicz criterion was also examined (for Scenario 1). When using the Hurwicz selection criterion, selection is performed based on the decision maker's 
optimism-pessimism index, $\alpha$. Depending on this preference, the rankings may come out different. Figure 5.4 displays the Hurwicz evaluation parameter, $P(\alpha)$, as a function of the decision maker's preference, $\alpha$, for this example.

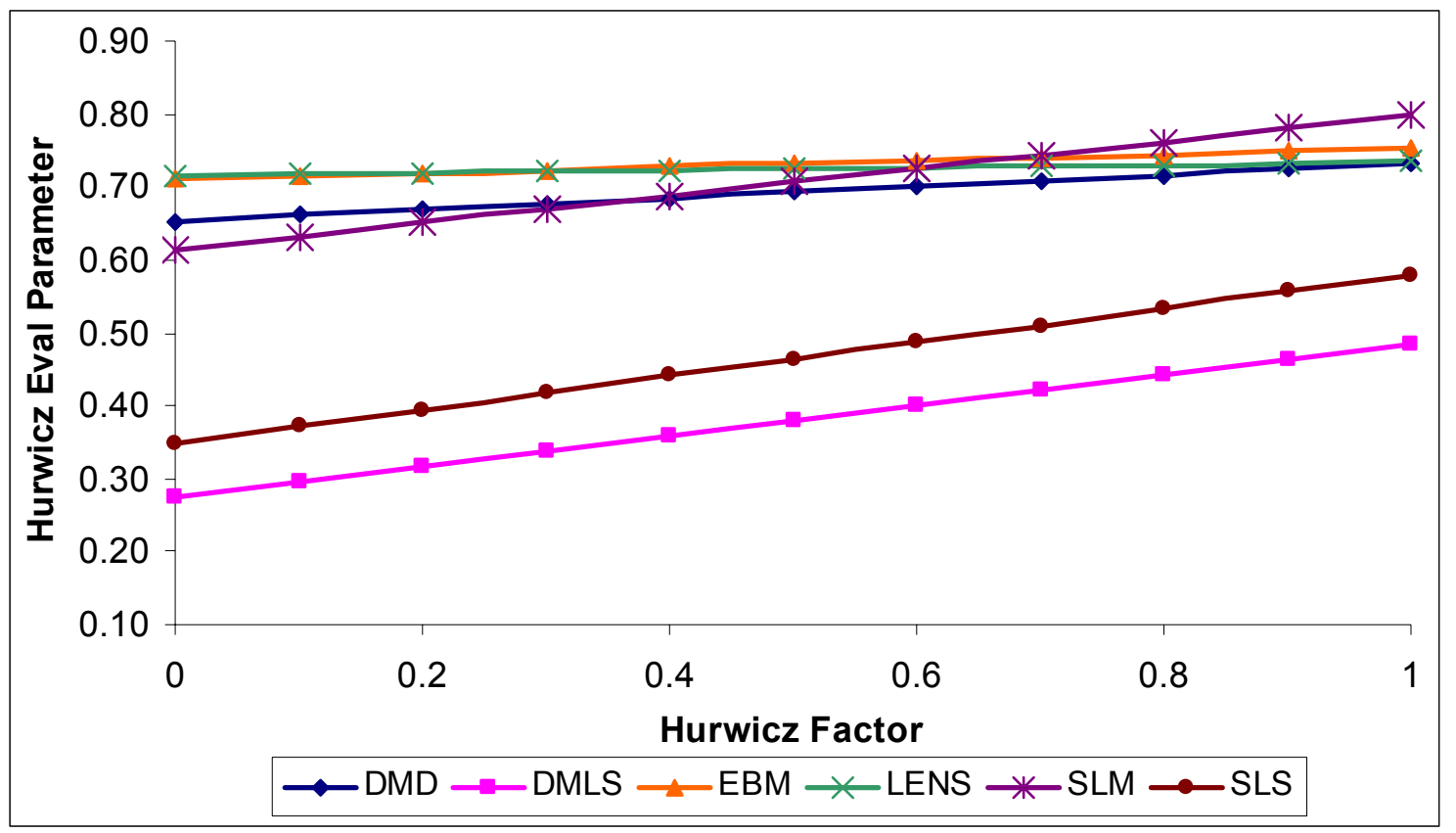

Figure 5. 4 Hurwicz evaluation parameter, $\boldsymbol{P}(\alpha)$, as a function of Hurwicz Factor, $\alpha$

As can be seen in Figure 5.4, the alternative rankings depend highly on the decisionmaker's decision preferences, $\alpha$. The top ranked alternatives, EBM and LENS, from Scenario 1 above are only ranked atop for the pessimistic decision maker. As the decision maker becomes more optimistic about the future, SLM becomes top ranked. Aside from SLM, EBM also increases in relative performance as the $\alpha$ increases. So what does this mean? This means that the decision maker must be as certain as possible in his/her assessment of his/her decision preference. If the decision maker is uncertain of his/her decision preference, a sensitivity study, such as the one performed in Figure 5.4, should be performed. This study will allow the decision maker to assure the rankings are insensitive to the uncertainty in his/her decision preferences. 
It should be noted that similar results can be expected from all parts within the volumetric range determined in Step 4 (all else equal). Based on our knowledge of the metal RM processes, the rankings seem in order, given the conditions specified in the example.

For comparison, the Selection DSP is performed in Section 5.1.5.

\subsubsection{The Selection DSP}

In this example, Selection DSP was used to select a RM technology for use by Albion. An average size caster wheel was used as a basis for selection. The dimensions are presented in Table 5.9.

Table 5. 9 Caster wheel dimensions

\begin{tabular}{|r|c|}
\cline { 2 - 2 } \multicolumn{1}{c|}{} & Dimensions \\
\hline Core Outer Diameter & 5 \\
\hline Core Inner Diameter & 4 \\
\hline Bore Outer Diameter & 2 \\
\hline Bore Inner Diameter & 1.25 \\
\hline Hub Length & 2.5 \\
\hline Core Outer Width & 1.75 \\
\hline Core Inner Width & 0.75 \\
\hline
\end{tabular}

As displayed in the table, the dimensions are scalar values due to the lack of uncertainty in this case.

\section{Step 1. Describe the alternatives and provide acronyms}

The alternatives are as follows: Direct Metal Laser Sintering (DMLS), Direct Metal Deposition (DMD), Electron Beam Melting (EBM), Laser Engineered Net Shaping (LENS), Selective Laser Sintering (SLS), and Selective Laser Melting (SLM). Descriptions are provided in Table 5.1.

Step 2. Describe each attribute, specify its relative importance and provide acronyms 
The attributes are as follows: UTS, Hardness, Density, Detail Capability, Geometric Complexity, Build Time, and Part Cost. Descriptions can be found in Section 5.1.4. The relative importances are displayed in Table 5.10.

Step 3. Specify scales, rate the alternatives with respect to each attribute.

The alternative ratings for this example are presented in Table 5.10. The build time and part cost were calculated for the respective technologies.

Table 5. 10 Attribute Ratings

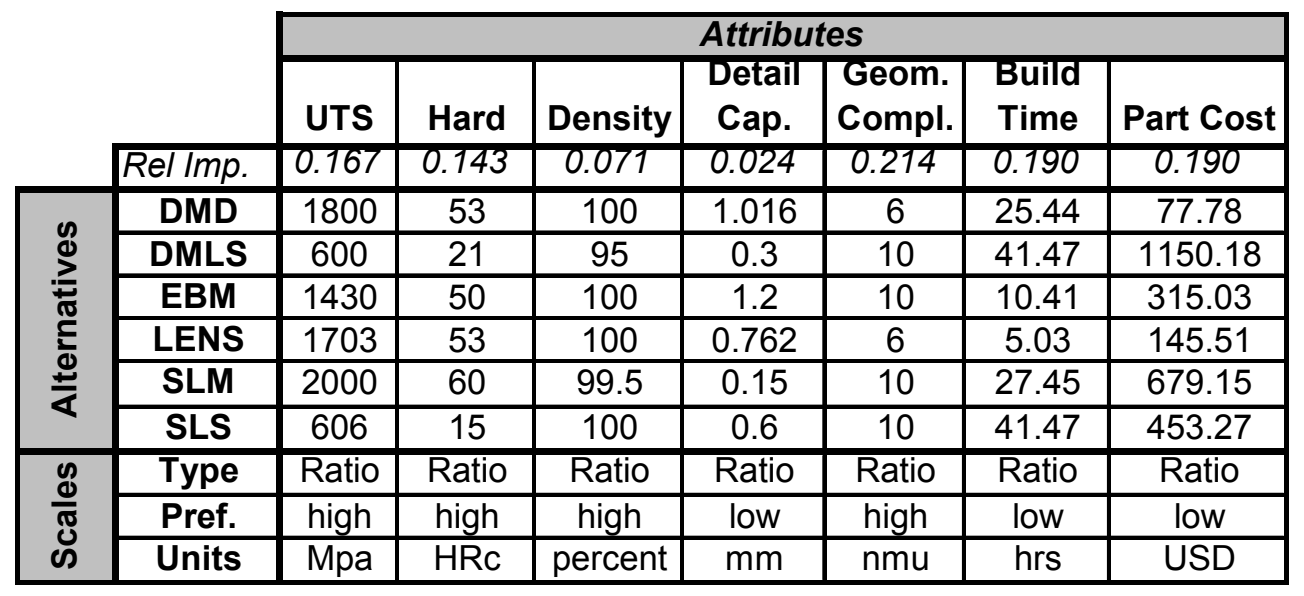

\section{Step 4. Normalize the attribute ratings}

The attribute ratings in Table 5.10 were normalized using the equations presented in Section 3.2.3.

\section{Step 5. Evaluate the merit function for each alternative}

The merit function values were calculated and are displayed in Table 5.11. 


\section{Table 5. 11 Merit Function Values}

\begin{tabular}{|c|c|c|}
\cline { 2 - 3 } \multicolumn{1}{c|}{} & $\begin{array}{c}\text { Merit } \\
\text { Function }\end{array}$ & Rank \\
\hline DMD & 0.61 & $\mathbf{4}$ \\
\hline DMLS & 0.25 & $\mathbf{6}$ \\
\hline EBM & 0.81 & $\mathbf{1}$ \\
\hline LENS & 0.70 & $\mathbf{3}$ \\
\hline SLM & 0.77 & $\mathbf{2}$ \\
\hline SLS & 0.42 & $\mathbf{5}$ \\
\hline
\end{tabular}

\section{Step 6. Post-Solution Analysis and Verification of results}

As seen in Table 5.11, EBM and SLM ranked atop the other alternatives. EBM was the top ranked alternative. This is largely due to the fact that a heavy importance weighting was given to geometric complexity, as well as build time and build cost. EBM and SLM both use powder beds, which favor production of overhangs, as well as having significantly greater volumetric build rates than the remaining alternatives.

\subsubsection{Comparison of Results Obtained}

When using Selection DSP for the selection of a RM technology for the production of custom caster wheels, an average size caster wheel was used to perform the selection. Because of this, the results (rankings) obtained are only valid for that average size part. One might make the assumption that this ranking, based on the average size part, is valid across the entire uncertainty interval, but would be flawed in doing so. For instance, let us look at Figure 5.5, where the merit value is plotted as a function of the uncertainty range. 


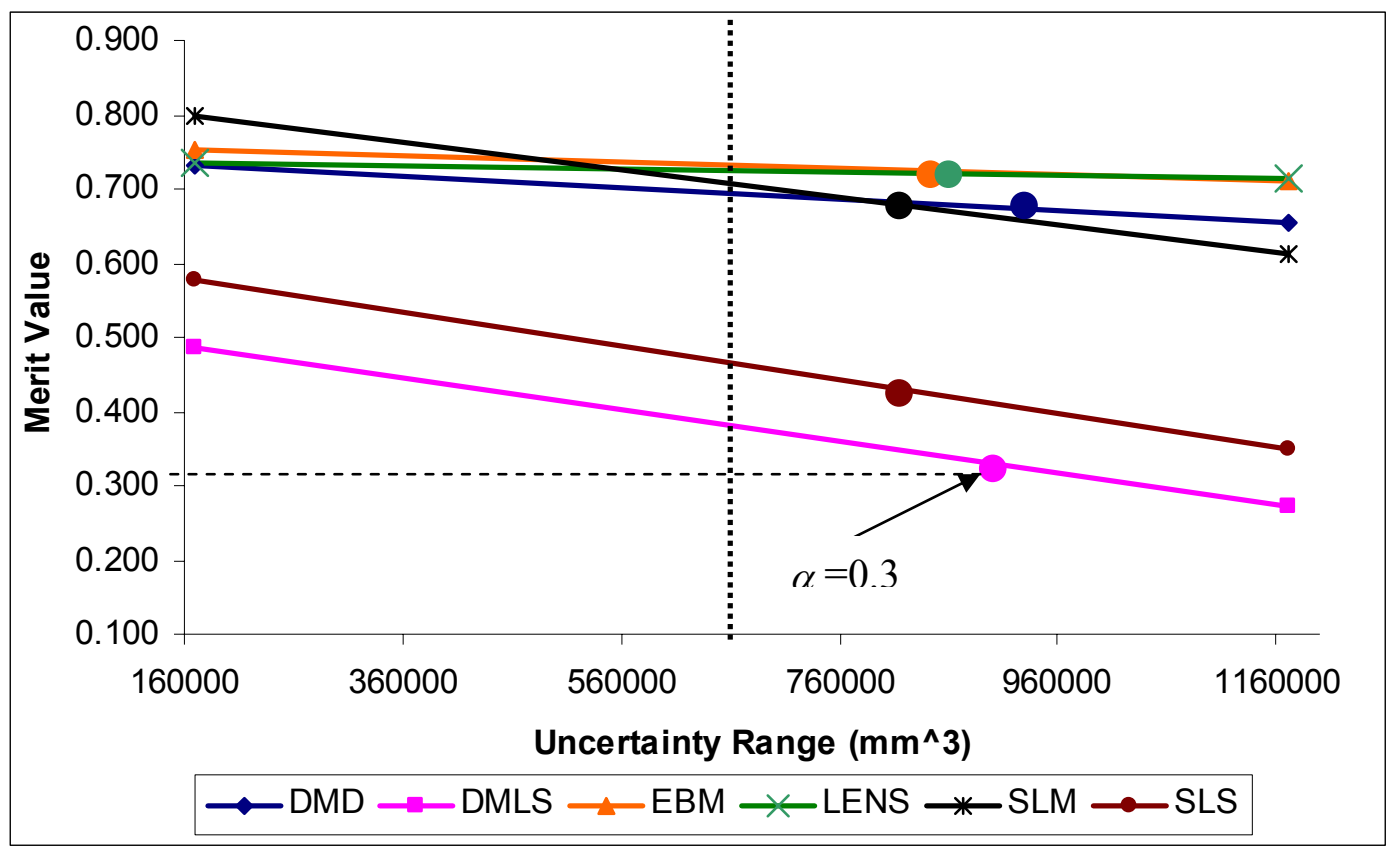

Figure 5. 5 Merit Value as a function of uncertainty

Selection DSP is performed at a single point in the uncertainty range of the part. For instance, in Figure 5.5, one would locate the point in the uncertainty range (whether average size part or some other) to perform the selection. For the selection DSP example in Section 5.1.5, selection was performed at the mark (vertical dotted line) displayed in the Figure 5.5, which is considered the average-size part. As can be seen in Figure 5.5, the alternative rankings differ greatly from one point in the uncertainty range to another. For instance, SLM, where a top rank is given at $160,000 \mathrm{~mm}^{3}$ in the size range, is ranked fourth at the maximum point in the size range, $1,160,000 \mathrm{~mm}^{3}$.

So how is this different from the selection method proposed in this thesis? Using Selection for RM, the decision maker has the ability to evaluate the alternatives based on their performance over the entire size range of the part. When using the Hurwicz selection criterion, selection is performed based on a point in the performance interval, as opposed to a point in the size range of the part. This point along the performance interval is the point that equates to the decision maker's decision preference, $\alpha$, and is calculated using the minimum and maximum performance states of the alternative. In this case, if the decision maker is pessimistic, he/she will evaluate the alternative based on its 
minimum range of performance. On the other hand, if the decision maker is optimistic, he/she will evaluate the alternatives based on their maximum range of performance. In this example, the decision maker was considered pessimistic, where $\alpha=0.3$, was used. This means the decision maker would rather evaluate the alternatives based on their minimum range of performance to assure that he/she is at least achieving some minimum level of performance.

In Figure 5.5, the point in the performance interval $(\alpha=0.3)$ by which each alternative is represented is displayed using a colored 'dot'. For example, DMLS has a Merit Function value of 0.34 , which corresponds to an $\alpha$ of 0.3 . This type of selection criterion allows the decision maker to evaluate the alternatives with respect to their performance ability, not the performance at a particular point in the uncertainty range of the part. Since $\alpha$ corresponds to a point in the uncertainty range, we can see that a decision preference of 0.3 correlates to larger part volumes (as seen by tracing the colored dots to the $\mathrm{X}$-axis). Although we have only considered part volumes of 800,000 to $940,00 \mathrm{~mm}^{3}$, this remains a more inclusive decision than only considering a single point in the size range of the part (as in the case of Selection DSP).

Instead of evaluating the alternatives based on a single point in the size range of the part (as in the case of Selection DSP), the Laplace criterion allows the decision maker to consider the entire size range of the part. By considering all the uncertainty states equally likely, this criterion allows the decision maker to evaluate the 'average' performance of the alternative. This is an added benefit over the Hurwicz criterion, since all performance states are considered, not just the maximum and minimum.

Another point of difference between the solution of the selection DSP and the Selection for RM is the manner in which the performance is calculated. When using selection DSP, the attribute ratings are normalized with respect to the lowest and highest rated alternatives (see Eqs. 2.1 and 2.2). This means that the performance (merit) of each alternative is evaluated with respect to the other alternatives. For instance, considering the UTS, SLM (rating of $2000 \mathrm{Mpa}$ ) was normalized with respect to the lowest rated 
alternative, DMLS (rating of $600 \mathrm{Mpa}$ ). When doing this, a true performance measure cannot be obtained. This type of normalization also skews the results since a very low performing alternative (DMLS) can make an average performer, such as EBM with a rating of $1430 \mathrm{Mpa}$, look promising because of its relation to the low performer.

In Selection for RM, the alternative ratings are normalized with respect to a given acceptable range (see Eqs. 3.1 and 3.2). This range is set by the decision-maker, and any value that underachieves this range is penalized by being assigned a merit of 0 , while overachievement is assigned the max value of 1 . By doing so, the performance of the alternatives can be evaluated with respect to the acceptable performance ranges that the decision maker has set forth for each attribute, as opposed to being evaluated with respect to each other. For instance, in this example, an acceptable range for UTS was given as $500-2500 \mathrm{Mpa}$. The attribute rating for SLM will be normalized with respect to that range, as opposed to being evaluated with respect to DMLS. This type of normalization scheme gives the decision maker the ability to evaluate the absolute performance of the alternatives.

\subsection{ILLUSTRATIVE EXAMPLE: DIRECT PRODUCTION OF HEARING AID SHELLS}

In this example, we consider the manufacture of custom hearing aid shells. This example is loosely based on an actual current product line produced by a collaboration between

Siemens and Phonak ${ }^{41}$. A rendering of a typical hearing aid shell is displayed in Figure 5.6 . 


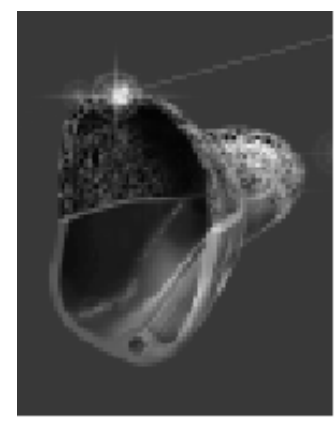

\section{Figure 5. 6 Hearing Aid Shell ${ }^{41}$}

As seen in the Figure 5.6, the hearing aid shell consists of an exterior geometry that is unique to the individual customer, as well as an internal void to house the internal components of the shell. Due to customization, each hearing aid will be different in a manner that is difficult to quantify parametrically. Because of this, we have chosen to represent the hearing aid as an elliptical cone, with the following parameters: major diameter, minor diameter, height, and wall thickness. A model of the hearing aid shell is displayed in Figure 5.7.

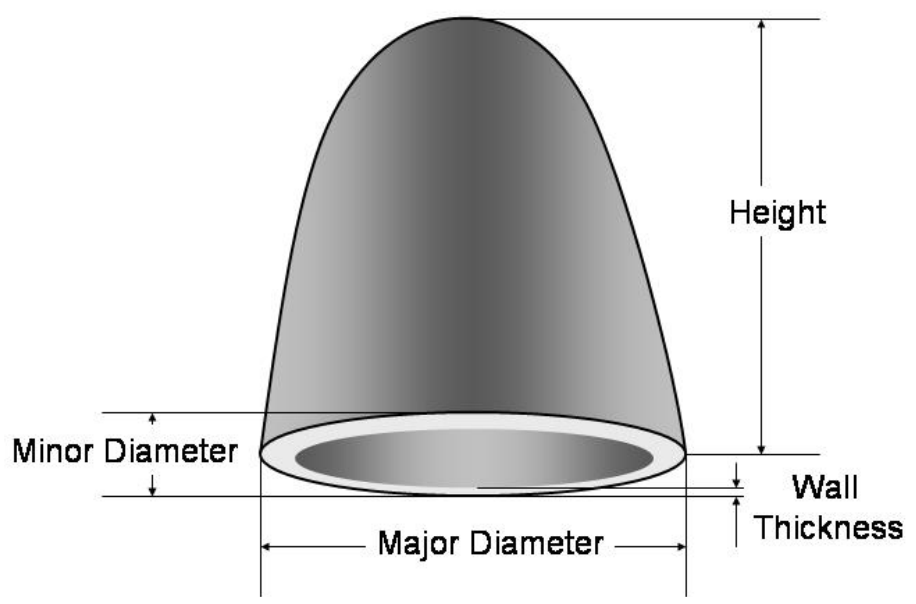

Figure 5. 7 Hearing Aid Shell Model

Since these hearing aid shells are custom, there is a considerable degree of uncertainty in each of the above parameters. 
Given the nature of the hearing aid business and competition, there is a need to be able to produce these quickly and cheaply, while also mimicking the quality exhibited by handmanufactured products. Given this need, most hearing aid companies already use RM to produce custom hearing aid shells. The author believes RM is a good candidate for hearing aid production for the following reasons:

- $\mathrm{RM}$ offers the ability to produce multiple custom hearing aid shell geometries in one build. Since each hearing aid is unique, production of a lot of these hearing aid shells significantly reduces the build time and cost, when compared to one-off production.

- RM does not require any special artisan services. With RM, the artifacts are manufactured directly from digital data. Because of this, no special artisans are needed for production of the custom parts.

- RM also allows the manufacturer to offer truly custom hearing aids. RM offers geometric complexity at no extra cost, whereas with traditional manufacturing process, the cost to manufacture the part increases as the complexity increases.

\subsubsection{RM Technologies}

In this example, the author will consider three RM technologies: Stereolithography (SLA), Selective Laser Sintering (SLS), and Fused Deposition Modeling (FDM). The details of these technologies, as well as the build time models, can be found in Sections 4.3.1-4.3.3.

\subsubsection{Selection for Rapid Manufacturing}

Before beginning the selection process, the uncertainty involved in the customization process was considered. Since these hearing aid shells will be customized, there is a degree of geometric uncertainty involved.

\section{Step 1. Characterize the uncertainty involved}


In this step, the range of customization is qualitatively defined. For this example, we decided to allow full customization of all the dimensions of the hearing aid shell, except the wall thickness, which is fixed at $1.1 \mathrm{~mm}$. The uncertainty is quantified using constraints and bounds on the dimensions of the hearing aid shells, displayed in Table 5.12 .

Table 5. 12 Hearing Aid Shell Dimensions

\begin{tabular}{|r|c|c|}
\cline { 2 - 3 } \multicolumn{1}{c|}{} & \multicolumn{2}{c|}{ Dimensions } \\
\cline { 2 - 3 } \multicolumn{1}{c|}{} & $\min$ & $\max$ \\
\hline major diameter & 13 & 18 \\
\hline minor diameter & 8 & 11 \\
\hline height & 16 & 22 \\
\hline thickness & 1.1 & 1.1 \\
\hline
\end{tabular}

\section{Step 2. Describe the alternatives and provide acronyms}

In this example, the alternatives are combinations of RM machines and materials. We chose three different RM technology groups: 3D Systems' Stereolithography (SLA 5000, SLA 7000, and SLA viper systems), 3D Systems' Selective Laser Sintering (Sinterstation HiQ system), and Stratasys' Fused Deposition Modelling (FDM Titan system). For the stereolithography (SLA) systems, Renshape SL5510, Renshape SL7560, DSM Somos 10120, and DSM Somos 9120 resins were used. For the selective laser sintering (SLS) systems, Duraform PA and Duraform GF powders were used. For the fused deposition modeling (FDM) system, ABS P400 was used.

\section{Step 3. Describe each relevant attribute, specify its relative importance and provide acronyms}

The attributes are described as follows:

Ultimate Tensile Strength (UTS): (see Section 5.1.4).

Young's Modulus (YM): YM is used to indicate the stiffness of the material.

Flexural Strength (FS): FS is the measure of a material's ability to resist bending.

Flexural Modulus (FM): FM is used to indicate the bending stiffness of the material.

Build Time (Time): (see Section 5.1.4).

Part Cost (Cost): (see Section 5.1.4). 
In this example, we examine two weighting scenarios (relative importance ratings). In Scenario 1, a pairwise comparison was used to determine relative importance of each attribute. In this scenario, build time and part cost were most heavily weighted because of their importance to the business structure surrounding customization of hearing aid shells. Flexural modulus was also highly weighted because of its direct impact on the customer. In Scenario 2, the attributes were given equal weightings. The relative importance weightings for each scenario are presented in Table 5.13.

\section{Step 4: Specify scales, acceptable range of values, and rate the alternatives with respect to each attribute.}

At this step, bounded geometric characteristics (such as part volume, area, etc.) are calculated using interval arithmetic operations on the bounded and constrained geometric dimensions. In our case, the particular geometric constraint of concern is the bounded part volume, which is used to calculate the build time and part cost in a build time estimation software package. The build time and part cost were calculated using the Build Time and Cost Estimation methods found in Chapter 4. The bounded part volume is $\left[115.3 \mathrm{~mm}^{3}, 224.9 \mathrm{~mm}^{3}\right]$.

The alternative ratings, as well as their acceptable performance ranges, are presented in Table 5.13. 
Table 5. 13 Attribute Ratings

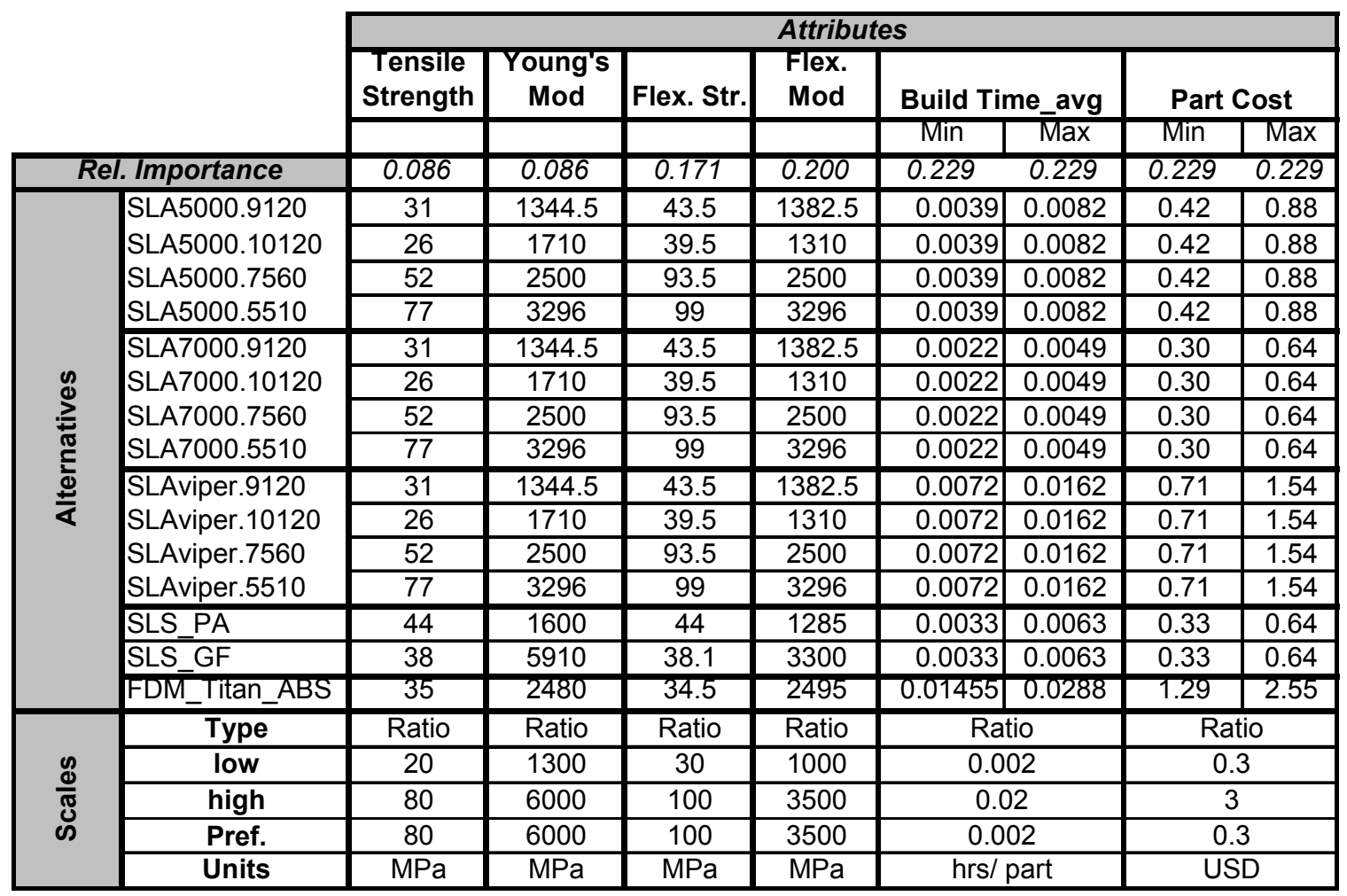

\section{Step 5: Normalize the attribute ratings}

The attribute ratings in Table 5.13 were normalized using the equations presented in

Section 3.2.3.

\section{Step 6: Rank and select the alternatives in order of preference}

The merit function values for Scenario 1 and Scenario 2 are displayed in Table 5.14. 
Table 5. 14 Merit Function Values for Scenarios 1 and 2

\begin{tabular}{|l|c|c|c|c|}
\cline { 2 - 5 } \multicolumn{1}{c|}{} & \multicolumn{2}{c|}{ Scenario 1 } & \multicolumn{2}{c|}{ Scenario 2} \\
\cline { 2 - 5 } \multicolumn{1}{c|}{} & min & max & min & max \\
\hline SLA5000.9120 & 0.512 & 0.438 & 0.404 & 0.350 \\
\hline SLA5000.10120 & 0.496 & 0.421 & 0.389 & 0.335 \\
\hline SLA5000.7560 & 0.775 & 0.700 & 0.697 & 0.643 \\
\hline SLA5000.5510 & 0.902 & 0.828 & 0.861 & 0.807 \\
\hline SLA7000.9120 & 0.535 & 0.485 & 0.422 & 0.385 \\
\hline SLA7000.10120 & 0.519 & 0.469 & 0.406 & 0.370 \\
\hline SLA7000.7560 & 0.798 & 0.748 & 0.715 & 0.678 \\
\hline SLA7000.5510 & 0.926 & 0.875 & 0.878 & 0.842 \\
\hline SLAviper.9120 & 0.461 & 0.317 & 0.367 & 0.262 \\
\hline SLAviper.10120 & 0.444 & 0.301 & 0.352 & 0.247 \\
\hline SLAviper.7560 & 0.723 & 0.580 & 0.660 & 0.555 \\
\hline SLAviper.5510 & 0.851 & 0.707 & 0.824 & 0.719 \\
\hline SLS_Duraf_PA & 0.541 & 0.490 & 0.454 & 0.417 \\
\hline SLS_Duraf_GF & 0.758 & 0.707 & 0.710 & 0.673 \\
\hline FDM_Titan_ABS & 0.444 & 0.221 & 0.391 & 0.229 \\
\hline
\end{tabular}

As seen in Table 5.13, there is overlap between the merit function intervals, therefore selection criteria must be used to rank the alternatives. The selection parameters for the selection criteria are displayed in Tables 5.15 and 5.16.

Table 5. 15 Selection Parameters for Scenario 1

\begin{tabular}{|l|c|c|c|c|}
\cline { 2 - 5 } \multicolumn{1}{c|}{} & Maximin & Maximax & $\begin{array}{c}\text { Hurwicz } \\
\mathbf{( 0 . 3 )}\end{array}$ & Laplace \\
\hline SLA5000.9120 & 0.44 & 0.51 & 0.46 & 0.47 \\
\hline SLA5000.10120 & 0.43 & 0.50 & 0.45 & 0.47 \\
\hline SLA5000.7560 & 0.70 & 0.77 & 0.72 & 0.74 \\
\hline SLA5000.5510 & 0.83 & 0.90 & 0.85 & 0.86 \\
\hline SLA7000.9120 & 0.48 & 0.54 & 0.50 & 0.51 \\
\hline SLA7000.10120 & 0.47 & 0.52 & 0.48 & 0.49 \\
\hline SLA7000.7560 & 0.75 & 0.80 & 0.76 & 0.77 \\
\hline SLA7000.5510 & 0.88 & 0.93 & 0.89 & 0.90 \\
\hline SLAviper.9120 & 0.32 & 0.46 & 0.36 & 0.39 \\
\hline SLAviper.10120 & 0.30 & 0.44 & 0.34 & 0.37 \\
\hline SLAviper.7560 & 0.58 & 0.72 & 0.62 & 0.65 \\
\hline SLAviper.5510 & 0.71 & 0.85 & 0.75 & 0.78 \\
\hline SLS_PA & 0.49 & 0.54 & 0.51 & 0.52 \\
\hline SLS_GF & 0.71 & 0.76 & 0.72 & 0.73 \\
\hline FDM_Titan_ABS & 0.22 & 0.44 & 0.29 & 0.33 \\
\hline
\end{tabular}


Table 5. 16 Selection parameters for Scenario 2

\begin{tabular}{|l|c|c|c|c|}
\cline { 2 - 5 } \multicolumn{1}{c|}{} & Maximin & Maximax & $\begin{array}{c}\text { Hurwicz } \\
\mathbf{( 0 . 3 )}\end{array}$ & Laplace \\
\hline SLA5000.9120 & 0.35 & 0.40 & 0.37 & 0.38 \\
\hline SLA5000.10120 & 0.34 & 0.39 & 0.35 & 0.36 \\
\hline SLA5000.7560 & 0.64 & 0.70 & 0.66 & 0.67 \\
\hline SLA5000.5510 & 0.81 & 0.86 & 0.82 & 0.83 \\
\hline SLA7000.9120 & 0.38 & 0.42 & 0.40 & 0.40 \\
\hline SLA7000.10120 & 0.37 & 0.41 & 0.38 & 0.39 \\
\hline SLA7000.7560 & 0.68 & 0.71 & 0.69 & 0.70 \\
\hline SLA7000.5510 & 0.84 & 0.88 & 0.85 & 0.86 \\
\hline SLAviper.9120 & 0.26 & 0.37 & 0.29 & 0.31 \\
\hline SLAviper.10120 & 0.25 & 0.35 & 0.28 & 0.30 \\
\hline SLAviper.7560 & 0.56 & 0.66 & 0.59 & 0.61 \\
\hline SLAviper.5510 & 0.72 & 0.82 & 0.75 & 0.77 \\
\hline SLS_PA & 0.42 & 0.45 & 0.43 & 0.44 \\
\hline SLS_GF & 0.67 & 0.71 & 0.68 & 0.69 \\
\hline FDM_Titan_ABS & 0.23 & 0.39 & 0.28 & 0.31 \\
\hline
\end{tabular}

As explained in Section 5.1.4, the Maximin and Maximax criteria can be seen as extreme cases of the decision maker's decision preference in the Hurwicz criterion, therefore, we will only consider the Hurwicz and Laplace criterion. A decision preference of 0.3 was determined from the lottery in Fig. 3.5 for the Hurwicz criterion. As explained in the previous example, in essence, we are deciding whether to evaluate the alternatives based on average performance in the case of Laplace criterion, or based on decision preference in the case of the Hurwicz criterion. We believe that a selection criterion should be chosen based on the type of decision problem. This is discussed further in the Section 5.3 of this chapter.

\section{Step 7: Post Solution Analysis and Verification of results}

As seen in Table 5.13, in comparing the results from the Hurwicz and Laplace criteria for Scenarios 1 and 2, SLA7000 using 5510 resin ranked atop the other alternatives, followed by SLA5000 using 5510 resin. This is mainly due to the superior material properties of the 5510 resin, as well as the high build speed and low part cost of the SLA 5000 and 7000 machines. In our example, the stereolithography machines seem to outperform the other technologies in most cases. As a whole, the rankings from the Hurwicz and Laplace criteria agree for both scenarios. 
As part of the sensitivity analysis, the effect of decision preference on the results of the Hurwicz criterion was also examined (for Scenario 1). When using the Hurwicz selection criterion, selection is performed based on the decision maker's decision preference, $\alpha$ (see Eq. 3.10). Depending on the decision maker's decision preference (or optimismpessimism index), the rankings may come out different. For this example, Figure 5.8 displays the Hurwicz evaluation parameter, $P(\alpha)$, graphed as a function of the decision maker's decision preference, $\alpha$.

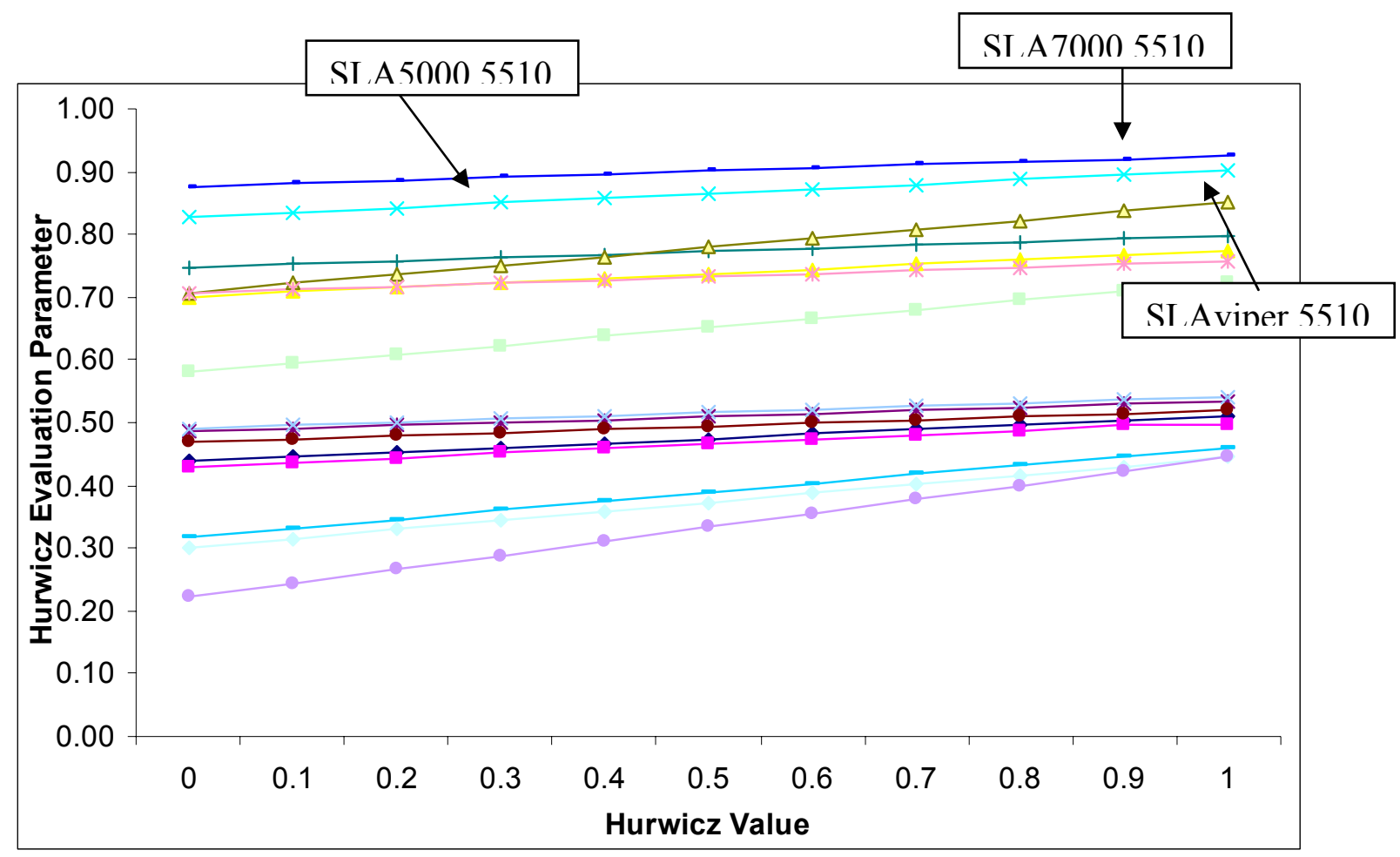

Figure 5. 8. Hurwicz evaluation parameter, $P(\alpha)$ as a function of Hurwicz Factor, $\alpha$

As can be seen in Figure 5.8, the alternative performance depends highly on the decision preference, $\alpha$. Although not as prevalent in this example, the alternative rankings do change as the decision maker becomes more optimistic in his/her preferences. In this case, it can be seen that the two top ranked alternatives, SLA7000 using 5510 resin and SLA5000 using 5510 resin, remain atop despite the decision maker's decision 
preferences. As the decision maker becomes more optimistic about the future, SLAviper using 5510 resin jumps from fifth to third rank. So what does this mean? As in the previous example, this means that the decision maker must be as certain as possible in his assessment of his decision preference. If the decision maker is uncertain of his/her decision preference, a sensitivity study, such as the one performed in Figure 5.8, should be performed. This study will allow the decision maker to assure the rankings are insensitive to the uncertainty in his/her decision preferences.

Based on our knowledge of the RM processes, the rankings seem in order, given the conditions specified in the example. For comparison, the Selection DSP is performed in Section 5.2.3.

\subsubsection{The Selection DSP}

For comparison, Selection DSP was also performed. In this example, an average size hearing aid shell was used. The dimensions are displayed in Table 5.17.

\section{Table 5. 17 Hearing Aid shell dimensions}

\begin{tabular}{|r|c|}
\cline { 2 - 2 } \multicolumn{1}{c|}{} & Dimensions \\
\hline major diameter & 15.5 \\
\hline minor diameter & 10.5 \\
\hline height & 18 \\
\hline thickness & 1.1 \\
\hline
\end{tabular}

As displayed in the table, the dimensions are scalar values due to the lack of uncertainty in this case.

\section{Step 1 Describe the alternatives and provide acronyms}

The alternatives are as follows: 3D Systems' Stereolithography (SLA 5000, SLA 7000, and SLA viper systems), 3D Systems' Selective Laser Sintering (Sinterstation HiQ system), and Stratasys' Fused Deposition Modelling (FDM Titan system). For the stereolithography (SLA) systems, Renshape SL5510, Renshape SL7560, DSM Somos 10120, and DSM Somos 9120 resins were used. For the selective laser sintering (SLS) 
systems, Duraform PA and Duraform GF powders were used. For the fused deposition modeling (FDM) system, ABS P400 was used.

\section{Step 2 Describe each attribute, specify its relative importance and provide acronyms}

The attributes are as follows: UTS, Young's Modulus, Flexural Strength, Flexural Modulus, Build Time, and Part cost. Descriptions can be found in Section 5.2.2. The relative importances are displayed in Table 5.18.

\section{Step 3 Specify scales, rate the alternatives with respect to each attribute.}

The alternative ratings for this example are presented in Table 5.18. The build time and part cost were calculated using the Build Time and Cost Estimation methods found in Chapter 4.

Table 5.18 Attribute Ratings

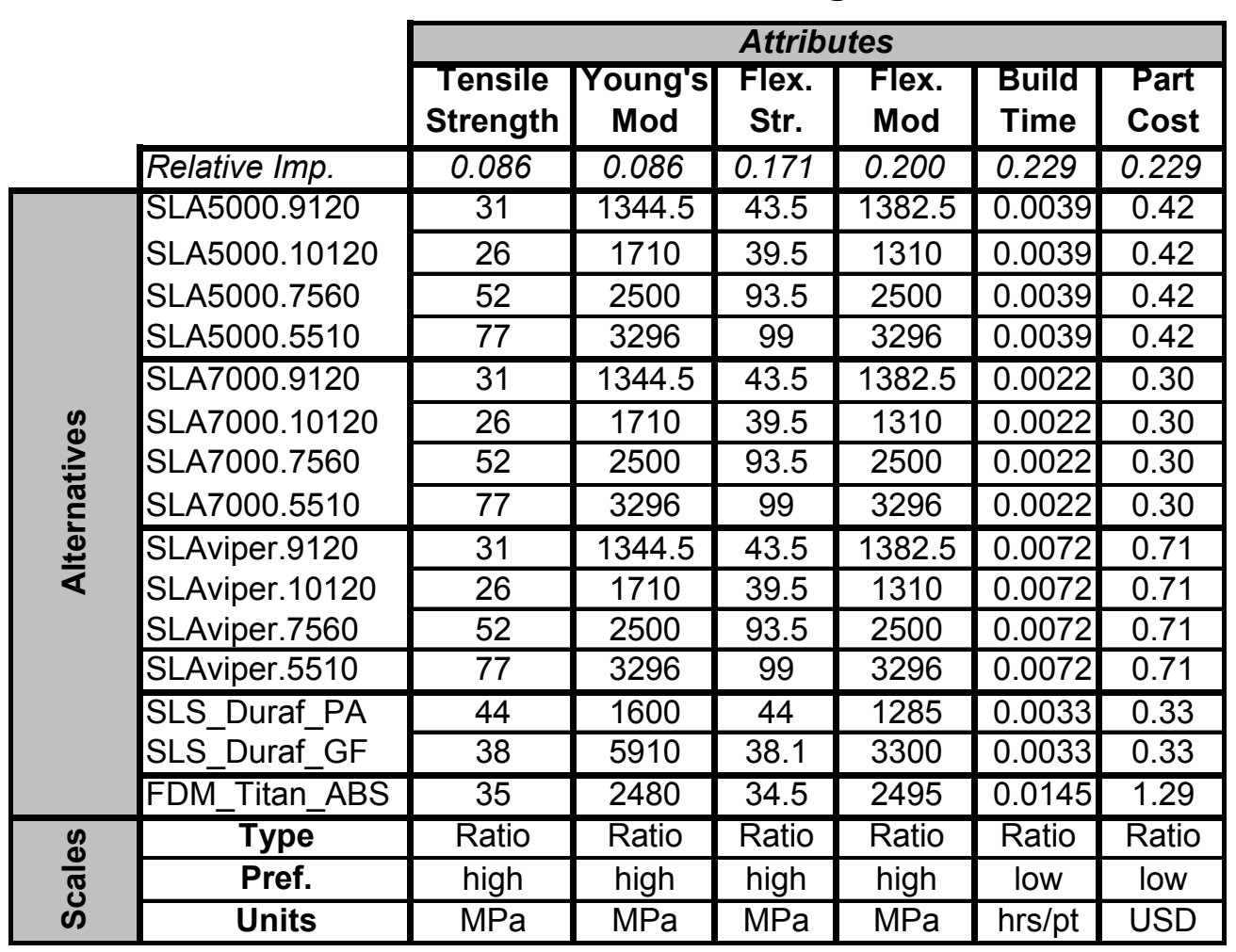

\section{Step 4 Normalize the attribute ratings}

The attribute ratings in Table 5.18 were normalized using the equations presented in Section 3.2.3. 


\section{Step 5 Evaluate the merit function for each alternative}

The merit function values were calculated and are displayed in Table 5.19.

Table 5. 19 Merit Function Values

\begin{tabular}{|l|c|}
\cline { 2 - 2 } \multicolumn{1}{c|}{} & $\begin{array}{c}\text { Merit } \\
\text { Function }\end{array}$ \\
\hline SLA5000.9120 & 0.44 \\
\hline SLA5000.10120 & 0.42 \\
\hline SLA5000.7560 & 0.74 \\
\hline SLA5000.5510 & 0.89 \\
\hline SLA7000.9120 & 0.50 \\
\hline SLA7000.10120 & 0.48 \\
\hline SLA7000.7560 & 0.80 \\
\hline SLA7000.5510 & 0.95 \\
\hline SLAviper.9120 & 0.31 \\
\hline SLAviper.10120 & 0.29 \\
\hline SLAviper.7560 & 0.61 \\
\hline SLAviper.5510 & 0.76 \\
\hline SLS_Duraf_PA & 0.49 \\
\hline SLS_Duraf_GF & 0.75 \\
\hline FDM_Titan_ABS & 0.16 \\
\hline
\end{tabular}

\section{Step 6 Post-Solution Analysis and Verification of results}

As seen in Table 5.18, SLA7000 using 5510 resin ranked atop the other alternatives, followed by SLA5000 using 5510 resin. This is mainly due to the high build speed and low part cost of the SLA 5000 and 7000 machines. The superior material properties of the 5510 resin are also a significant factor. In this example, as well as the example in Section 5.2.2, the stereolithography machines seem to outperform the other technologies. Based on our knowledge of the RM processes, the rankings seem in order, given the conditions specified in the example.

\subsubsection{Comparison of Results Obtained}

In Section 5.2.3, using selection DSP, an average size hearing aid shell was used for the selection of a RM technology for the production of custom hearing aid shells. As in the first example, the results (rankings) obtained are only valid for that point in the size 
range. One might make the assumption that this ranking, based on the average size part, is valid across the entire uncertainty interval. However, this would be assuming that the performance of the alternatives is constant along the entire size interval. For instance, let us look at Figure 5.9, where the merit value is plotted as a function of the uncertainty range.

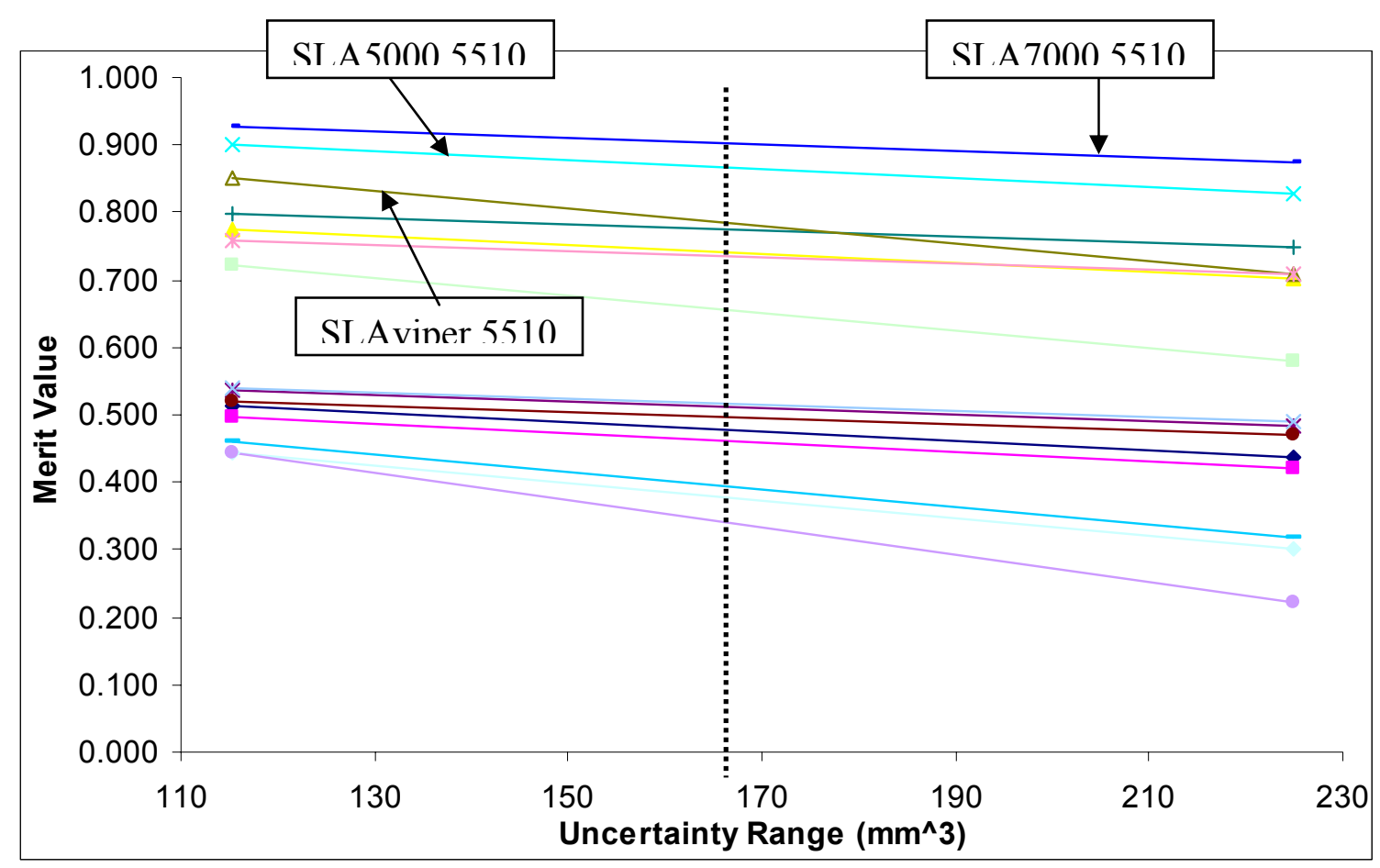

Figure 5.9 Merit Value as a function of uncertainty

Selection DSP is performed at a single point in the uncertainty range of the part. For instance, in Figure 5.9, one would locate the point in the uncertainty range (whether average size part or some other) to perform the selection. In Figure 5.9, selection was performed at the mark (dotted line) displayed in the figure. As can be seen in Figure 5.9, the alternative rankings differ greatly from one point in the uncertainty range to another. Although the top two alternatives (SLA7000 and SLA5000, both with 5510 resin) remain atop, SLA viper with 5510 resin changes ranking across the uncertainty interval.

So how is Selection DSP different from the selection method proposed in this thesis? As in the first example, when using the Hurwicz and Laplace selection criteria, selection is performed based on the entire size range of the part, as opposed to a point in the size 
range of the part. With the Hurwicz criterion, the alternatives are ranked based on a point in the performance interval of the alternative, which is determined by the decision maker's decision preference, $\alpha$, and the minimum and maximum performance states. In this example, the decision maker is considered pessimistic ( $\alpha=0.3)$, meaning he/she will evaluate the alternative based on its minimum range of performance. Using the Hurwicz criteria allows the decision maker to evaluate the alternatives with respect to their performance ability, not the performance at a particular point in the uncertainty range of the part, as in selection DSP. The Laplace criterion allows the decision maker to consider the entire size range of the part. By considering all the uncertainty states equally likely, this criterion allows the decision maker to evaluate the alternatives based on their 'average' performance.

Another point of difference between the solution of the selection DSP and the Selection for RM is the manner in which the performance (merit) is calculated. When using selection DSP, the attribute ratings are normalized with respect to the lowest and highest rated alternatives (see Eqs. 2.1 and 2.2). This means that the performance (merit) of each alternative is evaluated with respect to the other alternatives. For instance, when normalizing the attribute ratings of the top alternative (SLA7000.5510) with respect to the other alternatives, a normalized merit function value of 0.95 is obtained. This result infers that this performance of the technology is much higher than the absolute performance of 0.89 obtained using the normalization scheme proposed in this thesis. As seen in the first example, the normalization when using selection DSP skews the performance results by rating performance relative to the weakest alternative, whereas the normalization scheme proposed in this thesis calculates the performance based on an acceptable range obtained from the decision maker.

\subsection{ADDITIONAL DISCUSSION OF SELECTION FOR RM}

In addition to the comments made in Sections 5.1.6 and 5.2.4 about the behavior of the Selection for RM method, there are additional comments that are also worthy of nothing.

These comments will be discussed as follows: 


\section{Monotonicity}

As explained earlier, when using the Hurwicz selection criterion, the alternatives are represented by a point in the performance interval (merit function interval). It should be noted that when the monotonicity (with respect to the uncertainty range) of the alternatives is the same (either downward or upward sloping), Selection for RM will yield similar results as performing the selection DSP at a given point. When the monotonicities agree, the decision points are more clustered in the uncertainty interval. Depending on the degree of this clustering, the effects of uncertainty accounting may be lost, and Selection DSP (at a single point) can be used as a reasonable approximation.

\section{Deterministic Dominance}

In most cases of Selection for RM, the performance intervals of the alternatives will overlap. As explained earlier, in this case, one cannot definitively determine which alternative should be selected. By mapping the performance (merit) as a function of the geometric uncertainty range, we can establish dominance even in the case of overlapping intervals. If one alternative performs best at every state in the uncertainty range, as did SLA7000.5510 in Example 2, it is considered deterministically dominant, and can be chosen. In all other cases, one alternative cannot be considered deterministically dominant over the others. In these cases, although the selection criteria give us a basis for selection, the rankings should only be used as information to 'aid' the designer in selection.

\section{Interval arithmetic and computational expense}

Selection for RM, as a whole, can be considered computationally inexpensive on the grounds that intervals are very simple in nature and easy to propagate, compared with distributions. However, there are drawbacks to using interval analysis (arithmetic) to propagate the uncertainty in the selection process. As noted in Example 1, interval arithmetic, in its naïve formulation, gives a very conservative answer. This means that the bounds on the uncertainty can grow too large to be useful in the selection process. Therefore, care must be taken when propagating this uncertainty. It should be noted that 
there are methods to reduce this over-estimation of uncertainty when using interval arithmetic, since this over-estimation of the bounds can render the selection useless.

\section{Role of uncertainty}

The role of uncertainty in the Selection for RM method can be seen when plotting the performance intervals with respect to the geometric uncertainty (as displayed in the examples). In this plot, the effects of uncertainty can be determined by the rate at which the performance changes with respect to the uncertainty range. If the slope of the performance curve is equal to 0 , this denotes that uncertainty has no effect on the performance of the alternative. Whereas, as the affects of the uncertainty become larger on the performance, the slope of the performance curve increases (or decreases). This gives us a good assessment of the effects of considering uncertainty in the selection

process ${ }^{32}$. As explained earlier, with RM, it is typical for these curves to all have a negative slope, meaning performance decreases as the volume of the part increases, and vice versa. However, the different rates at which the slopes change for each alternative influences how much the ranking order of the alternatives change.

\subsection{DISCUSSION OF SELECTION CRITERIA}

As discussed earlier, because of the assumptions upon which the selection criteria are founded, the alternative rankings may be different. Now back to the question presented earlier in this thesis, "Which selection criteria should be used to select a RM technology under geometric uncertainty?" We believe that this choice is based on the type of decision problem considered. When using the Maximax and Maximin criteria, we evaluate each alternative based only on the maximum, or minimum, state of performance, while all other performance states are ignored. The Hurwicz criterion allows the decision maker to grade his/her decision, and use this grade to evaluate each alternative. The Laplace criterion evaluates the alternatives based on the average performance of the alternative over the uncertainty interval. Since the Maximax and Maximin criteria can be derived from the Hurwicz criterion, we will not consider them, specifically, in our discussion. 
In the context of selection for RM, the uncertainty range is defined by the range of products that are being offered. In this context, the Hurwicz criterion only considers the minimum and maximum performance states of a given alternative. A weighted sum, based on the decision preference of the decision maker, of the two states is used to rank the alternatives. By only considering the minimum and maximum performance states, the Hurwicz criterion ignores all other states of performance. On the other hand, the Laplace criterion considers all performance states. The Laplace criterion assumes an equal likelihood of all performance states to occur, therefore considering them equally and ranking the alternatives based on the average performance.

In the context of RM, we consider two general classes of decision problems: uniform and non-uniform product demand (or product forecast). In situations where a uniform demand for the products in the uncertainty range can be expected (they will be produced in equal amounts), the Laplace criterion can be used to rank the alternatives. This criterion is limited in the way in which it assumes this uniform demand, but does consider

all performance states. By considering all performance states, a better assessment of the overall performance of alternative is provided.

In situations of non-uniform demand, we cannot assume that all performance states in the geometric size range of the part are equally likely. In the case of the Laplace criterion, we consider the performance states equally likely since the demand of the products in the uncertainty range is equally likely. For non-uniform demand, we cannot consider the performance states equally likely and evaluate them as such. For these situations, we believe the Hurwicz criterion should be used, where the alternatives are ranked based on the decision maker's decision preference.

\subsection{CHAPTER SUMMARY AND VALIDATION}

In the first three chapters of this thesis, the background for and description of the Selection for RM methodology were presented. In this chapter, two illustrative examples 
of the use of Selection for RM were presented. In Section 5.1, an example of the direct production of custom, steel caster wheels was presented. In Section 5.2, an example of the production of custom hearing aid shells was also presented. The results of these selection processes were compared to the results obtained from using selection DSP on an average size part.

In this chapter, the Empirical Structural Validation (ESV) and Empirical Performance Validation (EPV) of the Selection for RM method have been established. As presented in Section 1.4.2, ESV involves building confidence in the 'appropriateness' of the example problems for illustrating and verifying the performance of the design method. As stated earlier, the caster wheel and hearing aid examples were taken directly from industry, where a need for customizing these products and selecting technologies suitable for providing this customization exists. Again, the method was established in the context of technology investment for custom manufacturing. Given the level of geometric uncertainty inherent to customizing caster wheels and hearing aid shells and the need for technology investment, both examples are considered directly applicable to the context upon which the Selection for RM method was established. Also, the simplicity of the examples provides an opportunity for us to focus on the uncertainty and how it is propagated in the selection process, as opposed to the complexity of the decision process.

As presented in Section 1.4.2, EPV is the evaluation of the 'usefulness' of the proposed method using example problems. To establish EPV, both of the examples presented in Chapter 5 were compared to a selection process (selection DSP), where geometric uncertainty is not considered. For both examples, this selection was performed using average-sized parts. The selection DSP for the custom caster wheel example was presented in Section 5.1.5 and the results compared in Section 5.1.6. The selection DSP for the custom hearing aid shell example was presented in Section 5.2.3 and the results compared in Section 5.2.4. In both cases, it was concluded that based on the results, it would be problematic to perform a selection based on the performance of an average size part, or any single part in the uncertainty range, as in the case of selection DSP. In 
contrast, it is better to perform the selection based on a point in the performance range of the part, depending on the decision maker's decision preferences.

The purpose of these examples was to show that the selection DSP can be extended to account for geometric uncertainty (Research Question \#1) and allow the designer to select an alternative under uncertainty (Research Question \#2). In the extended selection DSP, interval analysis was used to account for the geometric uncertainty inherent to customization. Also, the Hurwicz criterion (Decision Theory under strict uncertainty) was used to select an alternative under uncertain performance parameters. From the comparison of the results from the extended process with that of the traditional selection DSP, it is concluded that the inclusion of these extensions is appropriate and useful. These examples were also used to show the usefulness of the build time (example 2) and part cost (examples 1 and 2) presented in Chapter 4 of this thesis. 


\section{CHAPTER 6 CLOSURE AND CONTRIBUTIONS}

In this chapter, the research questions and their respective hypotheses will be revisited. The specific contributions to the body of knowledge on RM will also be reviewed in this chapter.

\subsection{REVISITING THE RESEARCH QUESTIONS}

As stated in Chapter 1, RM introduces the ability to provide customization opportunities. Coupled with this customization ability is uncertainty, which is mainly attributed to the lack of information about the customer's requirements and preferences. Given that, the author set out to answer the following primary research questions in this thesis:

"How can investment decisions be supported in the selection of a Rapid Manufacturing technology for customized products?"

To answer the primary research question, it was necessary to address several, more specific, research questions. The secondary research questions and hypotheses were as follows:

Question 1: How can the selection DSP be extended to account for the uncertainty associated with customization in the context of Rapid Manufacturing?

Hypothesis 1: By extending the selection DSP with interval accounting and analysis, the decision maker is able to consider the uncertainty associated with customization in the selection process.

Question 2: How can the selection DSP be extended to enable the designer to select a RM technology for investment under uncertainty? 
Hypothesis 2: By extending selection DSP with Decision Theory under strict uncertainty, the decision maker is able to select a technology, for investment, under uncertain parameters.

Question 3: How can part cost and build time be quantified for Rapid Manufacturing technologies with limited geometric information due to customization?

Hypothesis 3: Parametric build time and part cost models can be developed that depend explicitly on the parameters that characterize each technology and the overall part characteristics

Answering each of the above research questions involves the verification of the corresponding hypotheses. A brief review of how the hypotheses were verified is as follows:

Question 1 was answered through the presentation of the extended selection DSP, Selection for RM, in Chapters 3 and 5. In Chapter 5, Selection for RM, and subsequently, Hypothesis 1, was tested and verified using two example problems: direct production of custom caster wheels (Example 1) and production of custom hearing aid shells (Example 2). It was concluded that by extending the selection DSP with interval analysis and accounting, the decision maker was able to consider geometric uncertainty in the selection process.

Question 2 was also answered through the presentation of the extended selection DSP, Selection for RM, in Chapters 3 and 5. In Chapter 5, Selection for RM, and subsequently, Hypothesis 2, was tested and verified using two example problems. It was concluded that by extending the selection DSP with Decision Theory under strict uncertainty, the decision maker was able to select a technology under uncertainty. 
Question 3 was also answered through the presentation of the build time and cost estimation models in Chapters 4 and 5. In Chapter 4, Hypothesis 3 was tested and verified using quantitative and qualitative analysis. In Chapter 5, Hypothesis 3 was tested and verified using the example problems. It was concluded that the parametric build time and part cost estimation models could be developed that depend explicitly on the overall part geometry and technology characteristics.

A summary of this verification strategy for the hypotheses, including the test factors and test methods, is displayed in Table 6.1 .

Table 6. 1 Hypotheses Verification Outline

\begin{tabular}{|c|c|c|c|c|c|}
\hline Test Methods & 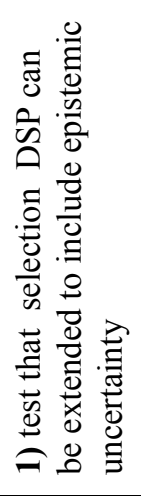 & 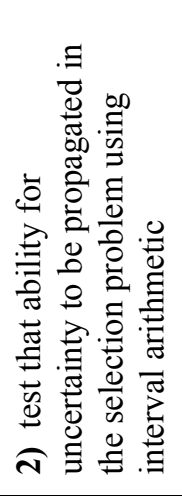 & 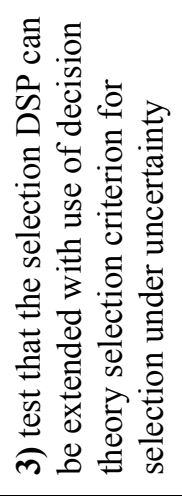 & 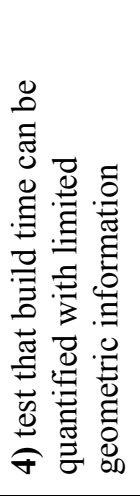 & 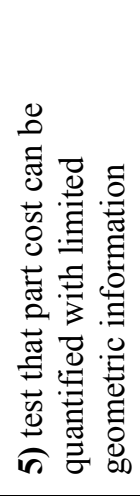 \\
\hline $\begin{array}{l}\text { Selection for RM } \\
\text { Theoretical Model } \\
\text { (Chapters } 2 \text { and 3) }\end{array}$ & $\mathbf{X}$ & $\mathbf{X}$ & $\mathbf{X}$ & & \\
\hline $\begin{array}{l}\text { Build Time and Cost } \\
\text { Model (mathematical } \\
\text { models) (Chapter 4) }\end{array}$ & & & & $\mathbf{X}$ & $\mathbf{X}$ \\
\hline $\begin{array}{l}\text { Example 1: Direct } \\
\text { production of custom, steel } \\
\text { caster wheels } \\
\text { (Chapter 5) }\end{array}$ & $\mathbf{X}$ & $\mathbf{X}$ & $\mathbf{X}$ & & $\mathbf{X}$ \\
\hline $\begin{array}{l}\text { Example 2: Direct } \\
\text { production of custom } \\
\text { hearing aid shells } \\
\text { (Chapter 5) }\end{array}$ & $\mathbf{X}$ & $\mathbf{X}$ & $\mathbf{X}$ & $\mathbf{X}$ & $\mathbf{X}$ \\
\hline
\end{tabular}

As seen in Table 6.1, each of the test factors for the hypotheses was thoroughly tested using the four different test methods. Each test factor was verified using multiple methods, therefore the hypotheses can be considered verified. 
Verification of these hypotheses was further brought together using the validation square, where the overall extended selection method, Selection for RM, was validated.

\subsection{VALIDATION AND VERIFICATION}

The validation strategy for this thesis is presented in Section 1.4. In this section, we revisit this validation strategy and briefly summarize the arguments. The last component of the validation square, Theoretical Performance Validity is also addressed in this section.

\section{Theoretical Structural Validation (TSV)}

TSV involves a two-part process, including checking the individual constructs and assumptions upon which the method is built, as well as checking the internal consistency of the method when combining the individual constructs. In Chapter 2, the first part of TSV was addressed, where each individual construct of Selection for RM was critically reviewed. The presented method is built upon three foundational constructs: selection DSP (Section 2.1), uncertainty handling (Section 2.2), and selection under uncertainty (Section 2.3).

The core focus of the work presented in this thesis involves the extension of the selection DSP methodology with uncertainty handling and support for selection under uncertainty. Selection DSP is introduced in Section 2.1.1, where its word formulation and steps for implementation are presented. This method is critically reviewed in Section 2.1.2, where its limitations are also addressed. The formal uncertainty handling formalisms are presented in Section 2.2. In this section, the two most prominent ways of representing geometric uncertainty, probability theory and interval analysis, are critically reviewed and their respective assumptions presented.

In Chapter 3, the second part of TSV is addressed, where the internal consistency of the presented method is addressed. As stated before, the core work in this thesis involves 
extending the selection DSP methodology to include uncertainty handling and support for selection under uncertainty. The extended selection DSP method, which is referred to as Selection for Rapid Manufacturing, is presented in Chapter 3. In Section 3.1, the context for which the selection method was established is presented. In Section 3.2, the Selection for Rapid Manufacturing under Uncertainty methodology, including the word formulation and steps for implementation, was presented. In this chapter, it was shown that these extensions to the selection DSP method did not cause any significant change in its formulation. Since the fundamental axioms of the interval analysis and the Hurwicz criterion remain intact, it was concluded that the resulting selection method was internally consistent.

\section{Empirical Structural Validation (ESV)}

ESV involves building confidence in the method's appropriateness. ESV is accomplished by showing that the example problems used are appropriate for the method proposed. Also, the data used in the example problem should be able to be used to support conclusions drawn.

In this thesis, ESV is addressed in Chapter 5, where two illustrative examples of rapid manufacturing are presented. In Section 5.1, an example of selection for the direct production of caster wheels is presented and in Section 5.2, an example of selection for the production of custom hearing aid shells. As stated in Section 3.1, the context for which the presented method was developed is technology investment for custom manufacturing. In their respective presentations, it was shown that both of the examples are applicable in this context.

\section{Empirical Performance Validation (EPV)}

EPV involves the evaluation of the 'usefulness' of the proposed method using example problems. In essence, EPV in this thesis involves showing that the extensions suggested for the selection DSP methodology are useful. EPV is also addressed in Chapter 5 of this

thesis. To address the 'usefulness' of the suggested extensions, the results from both of 
the illustrative examples presented in this thesis were compared to the results of the traditional selection DSP methodology using average-sized parts. In both cases, it was concluded that the extensions were indeed 'useful'. When comparing the results, the results from using the traditional selection DSP were based on the performance of that average-sized part, whereas the results from the extended selection DSP considered the overall performance of the machines as well as the decision maker's decision preferences. Given that, the results from the extended method yield a more robust solution.

Additionally, in Chapter 4, the build time and part cost estimation models developed for Selection for RM were compared to methods currently used in industry. Based on the performance of these models, it was concluded that the build time and cost models developed for this thesis were indeed 'useful' for the purposes of selection under uncertianty.

\section{Theoretical Performance Validation (TPV)}

TPV involves building confidence in the ability to extend the proposed method beyond the scope of the example problem to a general class of problems. The general class of problems in which this method is valid is defined by the following characteristics:

- Geometric uncertainty (can also deal with ranges of products, product families)

- Strict uncertainty - meaning no demand information known

- Technology investment - meaning the decision maker is selecting a technology for investment

Given that, this method can be extended beyond the realm of selection for rapid manufacturing into general realm of selection under epistemic uncertainty. As long as the uncertainty sources are epistemic and can be represented using intervals, the author has provided a method to propagate this uncertainty through the selection process as well as select under uncertain performance. 


\subsection{REVIEW OF RESEARCH GAP AND CONTRIBUTIONS}

Based on the review of the current approaches for selection under uncertainty in Section 1.2 , in the context of our decision problem, the following research gap needs to be addressed:

"Currently, there are no methods for considering geometric uncertainty (due to customization) in the selection of a RM technology for investment."

Based on this research gap, the completion of the work contained in this thesis has led to many significant contributions in the areas of selection of RM technologies and build time and part cost estimation for RM. Specifically, some key areas of contribution are:

1) uncertainty accounting

2) selection under uncertainty

3) performance evaluation

4) build time and part cost estimation

With respect to uncertainty accounting in the selection process, the selection DSP was extended to consider geometric uncertainty in the decision process. As explained in Chapter 2, selection DSP does not allow the inclusion of uncertainty in its problem formulation. In this thesis, we have presented a method for accounting (intervals) and propagating epistemic uncertainty (interval analysis) in the selection DSP. This gives the decision maker the ability to consider the entire size range (range of customization) of the part in the selection of a technology for investment. Additionally, since this method can be expanded to general cases of geometric uncertainty, the decision maker has the flexibility to account for entire product families or completely different parts in the selection process. With the traditional selection DSP, only one point in the size range of the part can be considered at a time.

Secondly, with respect to selection under uncertainty, the selection DSP has been extended to include the Hurwicz selection criteria for selection under epistemic 
uncertainty. As explained earlier, selection DSP offers no way of explicitly dealing with uncertainty. Given uncertain performance (merit function values), how does one select an alternative, especially in the case of overlapping performance intervals? While under these uncertainty conditions, the Hurwicz selection criteria allows the decision maker to select an alternative based on his/her decision preferences and the performance of the alternatives.

Additionally, the manner with which the performance of the alternatives is calculated has also been changed with the extended method. When using selection DSP, the attribute ratings are normalized with respect to the lowest and highest rated alternatives (see Eqs. 2.1 and 2.2). This means that the performance (merit) of each alternative is evaluated with respect to the other alternatives. With Selection for RM, we have offered an alternative normalization scheme, where the alternative ratings are normalized with respect to a range of acceptable performance values. This scheme allows the decision maker the ability to evaluate the alternatives based on absolute performance, not relative performance, as the selection DSP offers.

The work contained in this thesis also offers many contributions in the area of build time and part cost estimation for RM. In industry, build time and part cost estimation software available requires the use of a CAD model to estimate the build time and cost of parts. With the build time and part cost models presented in this thesis, only overall geometric parameters, such as bounding box and volume, are needed. The need for only these preliminary parameters allows the use of these models at any stage in the design and/or decision process. Additionally, these models offer the advantage of being parametric. Since the build time and part cost models are parametric, they can be adapted to any technology, assuming the information is available to characterize the machine. With respect to uncertainty accounting, these build and cost models can also be expanded to consider uncertainty in the geometric shape of the part. 


\subsection{RESEARCH LIMITATIONS AND FUTURE WORK}

Although there are several advantages to using the method proposed in this thesis, it does not come without its limitations. In this section, the main limitations to Selection for RM are discussed. These limitations open up avenues for future work, which is also discussed in this section.

The first limitation of Selection for RM relates to the types of uncertainty considered. As stated in Section 3.1, this project was scoped to only consider the geometric uncertainty inherent to mass customization. Although this thesis only considers geometric uncertainty, Selection for RM can be expanded to deal with other types of epistemic uncertainty, as long as they can be represented using intervals. The main limitation of this selection method is that it does not consider aleatory uncertainty, which is unavoidable in engineering design. Some examples of this type of uncertainty in engineering systems include uncertainty in material properties, material characteristics, machine characteristics, etc.

For a truly accurate accounting of uncertainty in the selection process, these types of uncertainty must be considered in the selection process. In the future, I believe this method can be extended for the accounting of both epistemic and aleatoric sources of uncertainty. Given the accounting of both types of uncertainty, methods of propagating both must also be developed. To perform selection, different selection criteria must also be used since the ones presented in this thesis are limited to cases of strict uncertainty.

Also dealing with uncertainty, another limitation to Selection for RM is that uncertainty in the decision maker's decision preferences is not considered. As shown with the examples in Chapter 5, in the case of the Hurwicz criterion, the alternative rankings greatly depend on the value selected for the decision preference. With that said, can the decision maker be totally certain about what his decision preference is? Given the metrics used, such as the certainty lottery, do these metrics accurately capture what the decision maker's decision preferences are for certain? I believe that use of a scalar value 
for this decision preference is flawed in the sense that it assumes complete certainty of the decision maker.

With respect to future work, I believe that better metrics can be developed to assess the decision maker's decision preferences. These metrics should include the accounting of the uncertainty associated with determining these decision preferences. This will be key in the advancement of uncertainty accounting in the selection process, especially as it relates to selection criterion where the decision maker's decision preferences are used as a basis for selection, such as the Hurwicz criterion.

The third significant limitation to Selection for RM is the manner in which the attribute ratings are normalized. Although the normalization scheme has its advantages, the assessment of the acceptable performance ranges can be problematic if the decision maker is not careful. The main limitation to this type of normalization scheme is the resolution at which the ratings are normalized. Depending on the scale of the acceptable range of performance, the normalized alternative ratings can be skewed. For example, lets consider Alternatives 1 and 2 with attribute ratings for cost of $\$ 5$ and $\$ 7$, respectively. In this example, we want to reduce cost. If the acceptable performance range is $\$[0,10]$, Alternatives 1 and 2 will receive normalized ratings of 0.5 and 0.3 , respectively. On the other hand, if the acceptable performance range is set to $\$[0,20]$, Alternatives 1 and 2 will receive normalized ratings of 0.75 and 0.65 , respectively. From this example, we can see how important accurate ranges are in the assessment of the merit functions.

The last limitation to the research presented in this thesis deals with the build time and cost estimation models proposed in Chapter 4. With respect to the build time and cost estimation models proposed in this thesis, the main limitation to this work deals with the parametric nature of the models. Since these models are parametric, the accuracy of the solution depends solely on the accuracy of the parameters. In the SLA, SLS, and FDM models presented, many assumptions were made for several parameters due to the limited amount of information available. Because of this, these estimation models should be 
used with caution until more accurate information is available to fully characterize these processes. However, depending on the level of accuracy needed, these models can be used for comparison studies.

In the future, a more accurate characterization of the RP machines will yield more accurate build time and cost estimates. This includes the collection of actual experimental data, as opposed to being forced to rely on company-quoted values, or assumptions.

\subsection{CLOSING REMARKS}

In closing, I present a few remarks on the 'value' of the selection method presented in this thesis, Selection for RM. As presented earlier, this thesis deals with the selection of a RM technology, for investment purposes, to manufacture customized products. The work presented in this thesis is focused on extending selection DSP to account for and select under the geometric uncertainty.

This question of concern in this closing section is, "What is the value of the work presented in this thesis to the working engineer?" Value can be defined as 'benefit' divided by 'cost'. In the context of selection processes, 'benefit' considers the added advantage to using Selection for RM and 'cost' considers such factors as computational expense of the method.

To the working engineer, the main benefit is that Selection for RM gives a way to consider the uncertainty that is inherent to customization in the selection process. This selection method allows the decision maker to consider the wide array of customized parts that can be built using RM in a single selection process. This provides a more robust solution than using a single-point evaluation.

With respect to computational expense, accounting for the geometric uncertainty in the context of customization reduces the time spent performing the selection. In other single- 
point selection methods that don't account for uncertainty, such as Selection DSP, a separate evaluation process is needed for each geometry being produced in the RM machine. With Selection for RM, a single selection process can be used to account for the entire size range of the part(s), thus reducing the time and computational expense of the selection.

As discussed earlier, value is benefit over cost. With the increased benefit of a robust selection, and reduced computational expense, Selection for RM can be seen as a valuable tool for considering geometric uncertainty in the selection process. Given the value exhibited with Selection for RM, I believe this work should be extended and research continued in this general field selection under uncertainty. 


\section{REFERENCES}

1. Tseng, M. and J. Jiao. Design for Mass Customization by Developing Product Family Architecture. in ASME Design for Manufacture Conference. 1998. Atlanta, GA.

2. Davis, S., From future perfect: Mass customizing. Planning Review, 1989. 17(2).

3. Silveira, G.D., D. Borenstein, and F.S. Fogliatto, Mass customization: Literature review and research directions. International Journal of Production Economics, 2001. 72: p. 1-13.

4. Hague, R., I. Campbell, and P. Dickens, Implications on design for rapid manufacturing. IMechE: Proc. Instn. Mech. Engrs, 2003. 217(C).

5. www.stereolithography.com. What is Rapid Prototyping? [cited 2005 5/20]; Available from: www.stereolithography.com/rapidprototyping.php.

6. $\quad$ Wohlers, T., Wohlers Report 2004: Rapid Prototyping, Tooling, \& Manufacturing State of the Industry. 2004.

7. myb2o, "The Production Paradigm". 2001.

8. Vadde, S., J.K. Allen, and F. Mistree, Catalog Design: Selection using available assets. Engineering Optimization, 1995. 25: p. 45-64.

9. $\quad$ Fernández, M.G., et al. Utility-Based Decision Support for Selection in Engineering Design. in 2001 ASME Design Engineering Technical Conference and Computers and Information in Engineering Conference. 2001. Pittsburgh, Pennsylvania: ASME.

10. Fernández, M.G., On Decision Support for Distributed Collaborative Design and Manufacture, in Mechanical Engineering. 2002, Georgia Institute of Technology: Atlanta, GA.

11. Reddy, R.P. and F. Mistree. Modeling Uncertainty in Selection using Exact Interval Arithmetic. in 1992 Design Theory and Methodology. 1992: ASME.

12. Pederson, K., et al. "Validating Design Methods and Research - The Validation Square". in ASME Design Theory and Methodology Conference. 2000. Baltimore, MD.

13. Williams, C.B., Platform Design for Customizable Products and Processes with Non-Uniform Demand, in Mechanical Engineering. 2003, Georgia Institute of Technology: Atlanta, GA.

14. Mistree, F., et al. Decision-Based Design: A Contemporary Paradigm for Ship Design. in Transactions, Society of Naval Architects and Marine Engineers. 1990. Jersey City, New Jersey.

15. Struble, C.L., et al. Compromise: a mulitiobjective hierarchical approach to the design of spacecraft thermal control systems. in ASME: Computers in Engineering Conference and Exhibit. 1989. Anaheim, CA.

16. Bascaran, E., R.B. Bannerot, and F. Mistree, Hierarchical Selection Decision Support Problems in Conceptual Design. Engineering Optimization, 1989. 14: p. 207-238.

17. Bae, H.-R., R.V. Grandhi, and R.A. Canfield, Epistemic uncertainty quantification techniques including evidence theory for large-scale structures. Computers \& Structures, 2004. 82: p. 1101-1112. 
18. Nikolaidis, E., D.M. Ghiocel, and S. Singhal, Engineering Design Reliability Handbook. 2005, Boca Raton, FL: CRC Press LLC.

19. Bae, H.-R., Uncertainty Quantification and Optimization of Structural Response using Evidence Theory, in Department of Mechanical and Materials Engineering. 2004, Wright State University. p. 222.

20. Wu, J.S., G.E. Apostolakis, and D. Okrent, Uncertainties in System Analysis: Probabilistic versus Nonprobabilistic Theories. Reliability Engineering and System Safety, 1990. 30: p. 163-181.

21. Zadeh, L.A., Fuzzy sets as a basis for a theory of possibility. Fuzzy Sets and Systems, 1978. 1: p. 3-28.

22. Shafer, G., A Mathematical Theory of Evidence. 1976, Princeton, NJ: Princeton University Press.

23. Rao, S.S. and L. Berke. Analysis of uncertain structural systems using interval analysis. in 37th AIAA/ASME/ASCE/AHS/ASC Structures, Structural Dynamics \& Materials Conference. 1996. Salt Lake City, UT.

24. Laplace, P.S., Essai Philosphique sur les Probabilites. 1825, Paris: Translation published by Dover, New York (1952).

25. French, S., Decision Theory: An Introduction to the Mathematics of Rationality. 1986, Chichester: Ellis Horwood Limited.

26. Zadeh, L.A., Fuzzy Sets. Information and Control, 1965. 8: p. 338-353.

27. Parsons, S. and A. Hunter, A Review of Uncertainty Handling Formalisms, in Lecture Notes in Computer Science. 1998. p. 8-37.

28. Dempster, A.P., Upper and lower probabilities induced by a multi-valued mapping. Annals of Mathematical Statistics, 1967. 38: p. 325-339.

29. H'egarat-Mascle, S.L., D. Richard, and C. Ottle, Multi-scale data fusion using Dempster-Shafer evidence theory. Integrated Computer-Aided Engineering, 2003. 10: p. 9-22.

30. Kangas, A.S. and J. Kangas, Probability, possibility, and evidence: approaches to consider risk and uncertainty in forestry decision analysis. Forest Policy and Economics, 2004. 6: p. 169-188.

31. Rao, S.S. and L. Cao, Optimum Design of Mechanical Systems Involving Interval Parameters. ASME Journal of Mechanical Engineering, 2002. 124: p. 465-472.

32. Moore, R.E., Interval Analysis. 1966, Englewood Cliffs, NJ: Prentice-Hall.

33. Ferson, S. and L.R. Ginzburg, Different methods are needed to propagate ignorance and variability. Reliability Engineering and System Safety, 1996. 54: p. 133-144.

34. Regan, H.M., M. Colyvan, and M.A. Burgman, A Taxonomy and Treatment of Uncertainty for Ecology and Conservation Biology. Ecological Applications, 2002. 12(2): p. 618-628.

35. Wald, A., Statistical Decision Functions. 1950 John Wiley.

36. Rapoport, A., Decision Theory and Decision Behavior: Normative and Descriptive Approaches. 1989, Dordrecht, The Netherlands: Kluwer Academic Publishers.

37. Hurwicz, L., Optimality criteria for decision making under ignorance, in Cowles Commision Discussion Paper No. 370 (mimeographed). 1951. 
38. Pham, D.T. and X. Wang, Prediction and reduction of build times for the Selective Laser Sintering process. Proceedings of the Institute of Mechanical Engineers, 2000. 214(B): p. 425-430.

39. www.3DSystems.com, 3D Systems, 3D Systems.

40. $\quad$ Sherman, L., Rapid Prototyping: Pretty Soon, You Won't Be Able To Get Along Without It. 2002.

41. Masters, M. Direct Manufacturing of Custom-Made Hearing Instruments. in SME Rapid Prototyping Conference. 2002. Cincinnati, OH. 